\title{
Multiple ionization and Coulomb explosion of molecules, molecular complexes, clusters and solid surfaces
}

\section{Tomoyuki Yatsuhashi, Nobuaki Nakashima}

\begin{tabular}{|c|l|}
\hline Citation & Journal of Photochemistry and Photobiology C: Photochemistry Reviews, $34 ; 52-84$ \\
\hline Issue Date & $2018-03$ \\
\hline Type & Journal Article \\
\hline Textversion & author \\
\hline \multirow{3}{*}{ Highlights } & $\begin{array}{l}\cdot \text { Molecular structure determinations by Coulomb explosion imaging are reviewed. } \\
\cdot \text { Intact multiply charged molecular cation productions are summarized. } \\
\text { ·Contribution of electrostatic interactions behind the ablation of solid materials is } \\
\text { introduced. }\end{array}$ \\
\hline \multirow{2}{*}{ Rights } & $\begin{array}{l}\text { C } 2018 \text { Elsevier B.V. This manuscript version is made available under the } \\
\text { This is the accepted manuscript version. The article has been published in final form } \\
\text { at https://doi.org/10.1016/j.jphotochemrev.2017.12.001 }\end{array}$ \\
\hline DOI & $10.1016 /$ j.jphotochemrev.2017.12.001 \\
\hline
\end{tabular}

\author{
Self-Archiving by Author(s) \\ Placed on: Osaka City University
}

YATSUHASHI, T., \& NAKASHIMA, N. (2018). Multiple ionization and Coulomb explosion of molecules, molecular complexes, clusters and solid surfaces. Journal of Photochemistry and Photobiology C: Photochemistry Reviews. 34, 52-84. doi: 10.1016/j.jphotochemrev.2017.12.001 
Multiple ionization and Coulomb explosion of molecules, molecular complexes, clusters and solid surfaces

\author{
Tomoyuki Yatsuhashi*, Nobuaki Nakashima
}

Department of Chemistry, Graduate School of Science, Osaka City University, 3-3-138 Sugimoto, Sumiyoshi, Osaka 558-8585 Japan

* Corresponding author.

E-mail address: tomo@sci.osaka-cu.ac.jp (T. Yatsuhashi)

\begin{abstract}
Intense femtosecond lasers as well as X-ray free electron lasers provide new means to produce multiply charged molecular cations. The fragmentation processes that these high energy species undergo, termed Coulomb explosion, are utilized to determine the static molecular structures as well as to trace the molecular dynamics of ultrafast chemical reactions. This review focuses on recent advances made in studies of Coulomb explosion imaging, highlighting the use of this process to determine the static structures of complex molecules, geometric isomers, chiral molecules and molecular complexes. Briefly, we summarize the recent time-resolved studies of surface electric fields and the controversy pertaining to the contribution of Coulomb explosion to the mechanism for ablation of solid surfaces.
\end{abstract}

Keywords: 
Coulomb explosion imaging; Electron deflectometry; Femtosecond laser; Multiply charged molecular cation; Tunneling; X-ray free electron laser

\section{Contents}

1. Introduction

2. Ionization and multiple ionization

2.1. Ionization in intense laser fields

2.2. Intact multiply charged molecular cations

3. Coulomb explosion

3.1. Observation of Coulomb explosion

3.2. Covariance mapping

3.3. Velocity map imaging

4. Coulomb explosion of molecules

4.1. Isolated molecules

4.1.1. Complex molecules

4.1.2. Geometric isomers

4.1.3. Enantiomers

4.2. Molecular complexes and clusters

4.2.1. van der Waals complexes

4.2.2. Hydrogen-bonded dimers

5. Coulomb explosion of solid surfaces

5.1. Time-resolved study of the electric fields

5.2. Carbon based materials 
6. Summary and perspectives

Acknowledgements

References

Abbreviations: BP, 3,5-dibromo-3',5'-difluoro-4'-cyanobiphenyl; CEI, Coulomb explosion imaging; COLTRIMS, cold target recoil-ion momentum spectroscopy; EI, electron ionization; MALDI, matrix-assisted laser desorption ionization; MCP, microchannel plate; MD, molecular dynamics; MMC, multiply charged molecular cation; PTFE, Polytetrafluoroethylene; REMPI, resonance enhanced multiphoton ionization; TOF, time-of-flight; TOF-MS, time-of-flight mass spectrometer; VMI, velocity map imaging; XFEL, X-ray free electron laser.

\section{Introduction}

The advent of intense femtosecond lasers opens up new avenue for generating attosecond light pulses [1, 2], visualizing wave functions [3-5], real-time imaging of molecules $[6,7]$ and controlling the dynamics of chemical reaction $[8,9]$. Moreover, these lasers have been used in a wide variety of analytical applications such as environmental sensing by filamentation $[10,11]$, inductively coupled plasma mass spectrometry [12] and laser desorption ionization imaging mass spectroscopy [13]. The advantageous features of femtosecond laser in processing solid materials are well known [14]. From a chemical viewpoint, the utilization of tailored intense laser pulses to control chemical reactions, such as promoting changes in the branching ratios of products, has had a strong impact $[8,15-17]$.

Strong electric fields emanating from high intensity laser distort potential energy 
surfaces of molecules followed by multiple electron ejection resulting in the formation of multiply charged molecular cations (MMCs). Strong Coulomb repulsions cause the MMCs to undergo rapid dissociation [18], which was termed "Coulomb explosion" by Carlson and White in 1966 [19]. When the charge numbers of MMCs are large enough, the energies released in Coulomb explosion are easily several tens of $\mathrm{eV}$, which is significantly more than typical chemical bonding energies. Coulomb explosion processes have been discussed for molecules [20] and clusters [21-23] in the gas phase. In contrast, Coulomb explosions of solids such as metals, dielectrics, semiconductors, polymers, and molecular crystals have been less emphasis even in recent reviews of the subject [24-27]. Recent studies have uncovered examples of Coulomb explosion of metal nanoparticles in the liquid phase [28-30], molecules in liquid helium droplets [31-33] as well as alkali metals in water [34].

Historically, MMCs have been produced by accelerating a molecular cation, for example $\mathrm{CH}_{4}{ }^{+}$, to within a few percent of the speed of light followed passage through a thin metal foil with a few nm thickness [35]. In this manner, the binding electrons are stripped away within 100 as, a time frame in which nuclear motion does not occur. As a result, MMCs have the same geometries as their molecular cation counterpart. Following stripping of binding electrons, the relative distances between the atoms in the original molecular cation expand as a consequence of Coulomb repulsive force between the similarly charged ionic centers in the MMCs. The expanded MMC is then projected on a detector plane (Fig. 1), thus, revealing the positions of the atomic ions which can be utilized to reconstruct the structure of the original molecular cation. By using this technique, called foil-induced Coulomb explosion imaging (CEI), identification of the structures of the enantiomers of the cation of trans-2,3-dideuterooxirane were elucidated 
in 2013 [36].

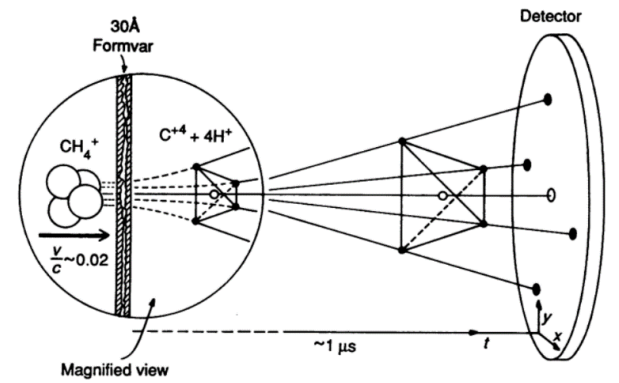

Fig. 1. Schematic of the Coulomb explosion imaging by thin-foil experiment. Reprinted with permission from Ref. [35]. Copyright 1989 AAAS.

Although thin-foil-induced CEI is a powerful method for structure determination, it is not applicable to neutral molecules. Another technique developed to produce MMCs involves collision with high energy projectiles, $\mathrm{Xe}^{20+}$ for example. The collision and ensuing charge transfer process takes place in only a few fs, but concern exists that within this short time frame the molecular fragmentation step is dependent on the energy of projectiles [37].

Carrying out of multiple ionization of atoms and molecules became a common process following the developments of intense pico- and femto-second lasers [38]. A variety of isolated molecules, molecular complexes, and molecular clusters have been transformed to the corresponding MMCs in gas phase upon exposure to intense ultrashort laser pulses. The most highly explored substrates for Coulomb explosion in 1990's were small molecules such as diatomic and triatomic molecules [20, 39, 40]. In contrast to use of the foil-induced and energetic projectile induced methods, Coulomb explosion promoted by using femtosecond laser pulses allows molecules to structurally deform especially along bond stretching coordinates because the interaction times are comparable 
to bond vibrational periods. Although some concern exists about deformations of molecular structures as a results of strong laser electric fields, identification of molecular structure by using CEI has been successfully carried out using femtosecond laser pulses.

Basically, Coulomb explosion can be considered as arising from the interplay between charged atoms under a point charge approximation. For example, the representative four-atom linear molecule, acetylene, possesses an elongated but still linear structure during Coulomb explosion. The resulting carbon ions are ejected along the laser polarization direction at $2.5 \times 10^{15} \mathrm{~W} \mathrm{~cm}^{-2}(790 \mathrm{~nm}, 130 \mathrm{fs})$ [41]. In cases where two charges of different masses exist within close proximity in the MMC, the light ion will be ejected while the heavy ion remains close to its original position to conserve momentum. If the heavy ion disturbs the direction in which light ions move, the light ions undergo structural deformation that enables them to move away. For example, heavy iodine atoms, which have a mass that is 11-times that of carbon, block the movement of lighter carbon ions in the Coulomb explosion of diiodoacetylene at $3.6 \times 10^{14} \mathrm{~W} \mathrm{~cm}^{-2}(800 \mathrm{~nm}, 45 \mathrm{fs})$ [42]. As a result, the angular distributions of carbon and hydrogen ions closely coincide in the case of acetylene, while carbon ions are ejected perpendicularly with respect to the iodine ones. The trajectories of ions following Coulomb explosion can be simulated by numerically solving the classical equation of motion under the assumption that each fragment ion is treated as a point charge and the repulsive forces between fragment ions are all Coulombic [43].

However, the dynamics of Coulomb explosion are strongly affected by electric fields and electron motions when much higher laser intensities are used. In 2000, we reviewed studies of Coulomb explosions of benzene and fullerene initiated by lasers above $10^{16} \mathrm{~W} \mathrm{~cm}^{-2}$ [18]. At this high laser intensity, field ionization, electron hopping, 
and a significant deformation of the molecular structure take place first followed by Coulomb explosion resulting in multiply charged atomic ions. The influence of laser electric fields on the ejection of carbon ions was demonstrated by using molecular dynamics (MD) simulations that considered ion-ion, electron-ion and electron-electron interactions. Although neutral fullerene has a spherical structure, our MD simulation studies show that the precursor ion has a rugby-ball-shape oriented along the laser polarization direction during the Coulomb explosion at $1 \times 10^{16} \mathrm{Wcm}^{-2}(800 \mathrm{~nm}, 120 \mathrm{fs})$ [44]. We also found that benzene deforms to form a ladder-like shape oriented the laser polarization direction during Coulomb explosion at $8.0 \times 10^{16} \mathrm{~W} \mathrm{~cm}^{-2}(800 \mathrm{~nm}, 120 \mathrm{fs})$ (Fig. 2) [45]. Consequently, it is known that molecular deformations caused by strong laser electric fields determine the ejection behavior of atomic ions. Furthermore, the deformation is more significant in the case of $\mathrm{C}_{6} \mathrm{~F}_{6}\left(800 \mathrm{~nm}, 130 \mathrm{fs}, 1 \times 10^{16} \mathrm{Wcm}^{-2}\right)$, in which the fluorine atoms have a mass that is 19 times that of hydrogen [46].

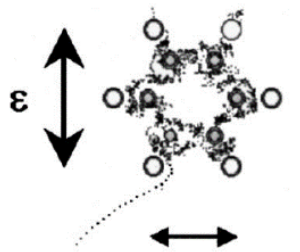

$5 \AA$

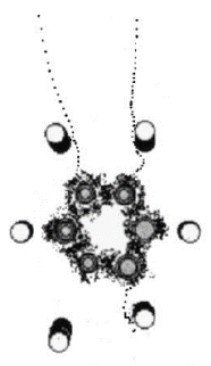

(b) $14.7 \mathrm{fs}$

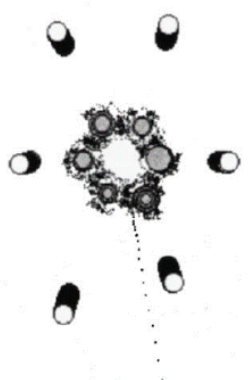

(c) $18.2 \mathrm{fs}$

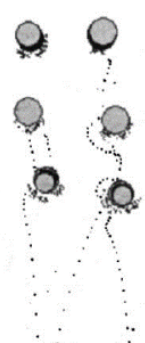

(d) $31.7 \mathrm{fs}$

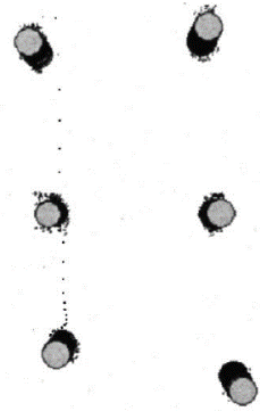

(e) $43.6 \mathrm{fs}$

Fig. 2. Simulation of Coulomb explosion dynamics of benzene at $8.0 \times 10^{16} \mathrm{~W} \mathrm{~cm}^{-2}$. The arrow shows the direction of the laser electric fields. Open circles indicate $\mathrm{H}^{+}$. The smallest gray circles indicate $\mathrm{C}$ and the largest ones $\mathrm{C}^{4+}$. The dots present electron trajectories. Reprinted with permission from Ref. [45]. Copyright 2002 AIP Publishing 
LLC.

From our viewpoint, multiply charged cationic species can be divided into three groups according to their charge number and stability. In this manner, they are composed of groups distinguished as dications, short-lived MMCs, and intact MMC. Coulomb explosion of dications, often called a charge separation process, has been extensively studied by using electron ionization (EI) mass spectrometry [47]. Recently, the chemical reactions of dications have risen to an important position in intense laser chemistry because the yields of dications are greatly enhanced by using femtosecond laser ionization [48-54]. Here we would like to define short-lived MMCs as species that are not detected by using time-of-flight mass spectrometer (TOF-MS). In other words, short-lived MMCs have lifetime that are shorter than a few hundred ns, which is a typical acceleration time in the TOF-MS. We will discuss about the CEI of short-lived MMCs in the following sections. On the contrary, intact MMCs are definitively identified by the mass-to-charge ratio, isotope pattern, and narrower peak width than those of lower-charged ions by using TOF-MS. The lifetime of intact MMCs are longer than a few hundred ns, for example, diiodoacetylene tetracation does not decay completely at least for $11 \mu \mathrm{s}$, which is the flight time from the ion source to the entrance of the second mass analyzer (reflectron) in TOF-MS [55]. In contrast to Coulomb explosions of short-lived MMCs, the chemistry of intact MMCs have been only rarely explored (see Section 2.2). Despite this, the generation of intact MMCs, which are a new class of chemical species, is of current interest to those studying high-energy chemistry, photochemistry, interstellar chemistry, particle beam therapy and spin chemistry [47, 56-64].

The discussion in this review focusses on Coulomb explosions of molecules, 
molecular complexes, clusters and solid surfaces that are induced by femtosecond laser pulses and, in part, by X-ray radiations from synchrotron source or X-ray free electron lasers (XFELs). In the next section, the mechanism for ionization of these substrates in intense laser fields is described, together with a short introduction of intact MMCs. In Section 3, we describe the detection of Coulomb explosion events while in Section 4 the details of Coulomb explosions of isolated molecules, molecular complexes and clusters are introduced along with highlights of the identification of molecular structures by using CEI. Recent developments arising from time-resolved studies of electric fields on solid surfaces along with the relationship between Coulomb explosions and mechanism for ablation of solid surface are presented in Section 5. Finally, a summary of studies in this field and perspective for future efforts are provided in Section 6.

\section{Ionization and multiple ionization}

\subsection{Ionization in intense laser fields}

Ionization is a fundamental and primary process in photochemistry. The first ionization potentials of organic molecules typically fall in the 6-12 eV region. Single photon ionization has been carried out by using vacuum ultraviolet light emitted from a rare gas lamp, for example those composed of helium $(58.4 \mathrm{~nm}, 21.2 \mathrm{eV})$ and Krypton $(117 \mathrm{~nm}, 10.6 \mathrm{eV})$, and/or by using a synchrotron light sources. In contrast, the shortest wavelength of commercially available pulsed laser is $157.6 \mathrm{~nm}(7.87 \mathrm{eV})$; therefore, more than two photons are required to ionize molecules by using conventional lasers. Resonance enhanced multiphoton ionization (REMPI) in combination with supersonic jet techniques has been utilized to distinguish molecules of similar structure $[65,66]$. As the intensity of the laser pulse increases, nonresonant multiphoton ionization, corresponding 
to simultaneous multiple photon absorption by one atom or molecule, occurs. The nonselective nature of nonresonant multiphoton ionization is an ideal feature when the technique is utilized in time-resolved photochemical studies [67-71].

Yamanouchi described the processes that occur in intense laser field in terms of light-matter interactions that take place in the Coulombic and relativistic intensity regimes (Fig. 3) [72]. In the Coulombic regime, strong laser electric fields perturb the motion and structure of a molecule causing molecular alignment and deformation. Moreover, multiple electron ejection caused by the field (tunnel) ionization is followed by Coulomb explosion. X-ray and high-energy particles are produced and nuclear fusion reactions occur in the relativistic regime.

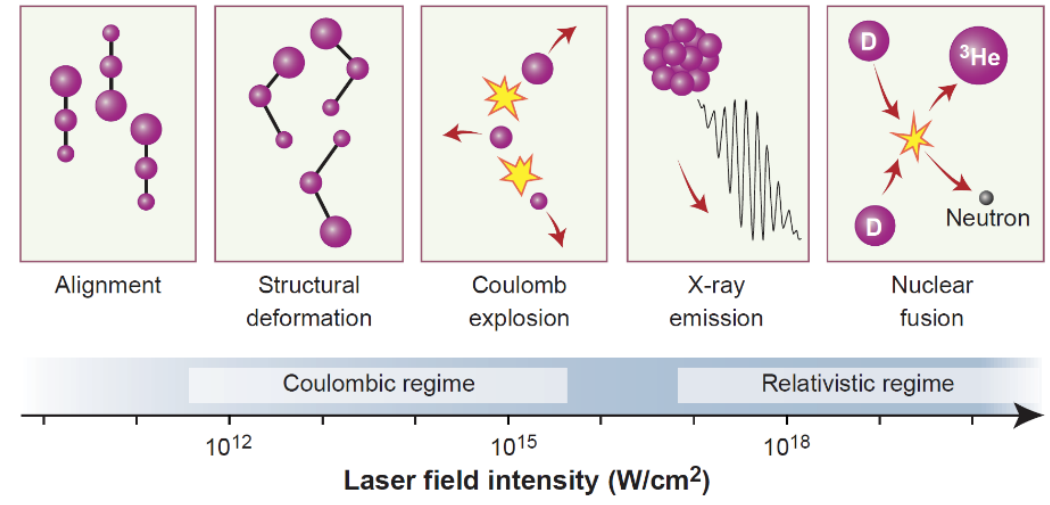

Fig. 3. Behaviors of molecules and clusters in intense laser fields. Reprinted with permission from Ref. [72]. Copyright 2002 AAAS.

It is important to consider how ionization of atoms and molecules take place within the Coulombic regime. A number of ionization schemes have been proposed including above-threshold [73], tunnel (sequential) [74], barrier suppression (over the barrier) [75] and nonsequential ionization (electron rescattering) [76]. Laser intensities of $10^{13}$ to $10^{15} \mathrm{~W} \mathrm{~cm}^{-2}$ correspond to electric field strengths of $10^{8}-10^{9} \mathrm{~V} \mathrm{~cm}^{-1}$, which are 
comparable to those used in the field ionization method (ca. $10^{7} \mathrm{~V} \mathrm{~cm}^{-1}$ ) employed in mass spectrometry and the atom probe method (ca. $10^{8} \mathrm{~V} \mathrm{~cm}^{-1}$ ). Supposing that a hydrogen atom is exposed to these strong electric fields, the potential of a hydrogen atom can be expressed by equation 1 ,

$\mathrm{V}(r, t)=-\frac{e^{2}}{4 \pi \varepsilon_{0}} \frac{1}{r}+e E(t) r$

where $\mathrm{V}(r, t), e, \varepsilon_{0}$, and $E(t)$ are Coulomb potential (in $\mathrm{J}$ unit) depending of nuclear coordinate $r$ (in $\mathrm{m}$ unit) and time $t$ (in $\mathrm{s}$ unit), elemental charge (in $\mathrm{C}$ unit), vacuum permittivity (in $\mathrm{C}^{2} \mathrm{~J}^{-1} \mathrm{~m}^{-1}$ unit), and electric field strength (in $\mathrm{V} \mathrm{m}^{-1}$ or $\mathrm{N} \mathrm{C}^{-1}$ unit), respectively. In Figure 4 is shown a schematic of the potential energy curve for a hydrogen atom exposed to an electric field $\left(1.9 \times 10^{11} \mathrm{~V} \mathrm{~m}^{-1}\right)$, which is correspond to laser intensity of $5 \times 10^{15} \mathrm{~W} \mathrm{~cm}^{-2}$. Before the external field is applied, the electron in the Coulomb potential well, where the Coulomb field of a hydrogen atom is $5.1 \times 10^{11} \mathrm{~V} \mathrm{~m}^{-1}$, moves back and forth along a nuclear coordinate $r$. When the external field is applied, the electron would escape from the potential barrier by tunneling. In the case of molecules in intense laser fields, many interesting and molecular specific phenomena such as Coulomb explosion [77], bond softening [78], charge resonance enhanced ionization [79-82], above-threshold dissociation [83], and orientation-selected ionization [84] can occur. 


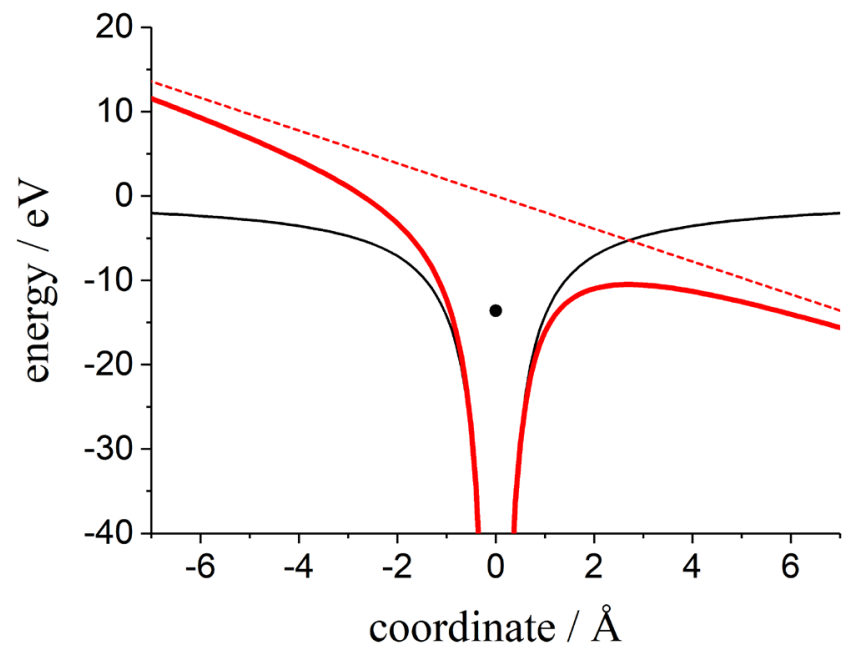

Fig. 4. The potential energy curves of a hydrogen atom with (bold red line) and without external electric fields (solid black line). The dashed red line indicates the external electric field $\left(1.9 \times 10^{11} \mathrm{~V} \mathrm{~m}^{-1}, 5 \times 10^{15} \mathrm{~W} \mathrm{~cm}^{-2}\right)$.

One of the important findings arising from studies of intense laser field chemistry is that intact molecular cation radicals as well as intact MMCs are generated. The former is utilized as a soft-ionization method to elucidate the molecular mass of molecules while the latter has opened a new research field because intact organic MMCs cannot be generated in abundance by any other methods. The possibility of soft-ionization, i.e. nearly fragment-free ionization by using femtosecond laser pulses, was proposed in 1995 by three groups [85-87], and the advantages of femtosecond laser pulses were reviewed by Ledingham and Singhal in 1997 [88]. However, it was found that femtosecond laser pulses do not always produce intact molecular cation radicals but sometimes cause heavy fragmentations of substrates. Many attempts were made to investigate the factors governing intact molecular cation radical formation and fragmentation in ionization [8992] or post ionization processes [18, 71, 93-101]. Regardless of the method of generation, fragmentation is expected to take place for electronically excited molecular cation radials 
as a consequence of repulsive interactions. The possible modes for production of electronically excited states of molecular cation radicals include nonadiabatic excitation process [89-92], ejection of an electron from low-lying molecular orbital [102-106] and resonant excitation of a molecular cation radical by the tailing edge of the laser pulse [18, 93-98]. Supposing that an electron in molecule adiabatically responds to a laser electric field, the likelihood and nature of ionization will be independent of the ionization wavelength. On the contrary, the quasi-static scenario fails if an electron cannot follow the oscillating laser electric field. In this nonadiabatic case, electron dynamics is ionization-wavelength and molecular-length dependent. According to these considerations, fragmentation is expected to occur when shorter (high frequency) wavelength light and longer (larger) molecules are employed. Nonadiabatic (multi)electron dynamics has been used successfully to explain many interesting phenomena, including fragmentation reactions, in the field of ultrafast intense laser science [107]. However, fragmentation of large molecules is not a straightforward process. Fragmentation is not always dominant at shorter wavelength but it is at longer wavelength: for example, $\mathrm{Fe}(\mathrm{CO})_{5}$ dissociates at $0.8 \mu \mathrm{m}$ but stays intact at $1.35 \mu \mathrm{m}$, whereas for $\mathrm{Ni}(\mathrm{CO})_{4}$ fragmentation is higher at $1.35 \mu \mathrm{m}$ than at $0.8 \mu \mathrm{m} ; \mathrm{Cr}(\mathrm{CO})_{6}$ shows little fragmentation at both wavelengths [108]. Moreover, the yield of specific fragment ion is maximized at the specific ionization wavelength [109-114]. In addition, the molecules having similar size show significantly different fragmentation behavior $[93,96$, 98]. These observations indicate that phenomena that cannot be resolved by using the nonadiabatic scenario have been simply explained by separately treating the ionization and dissociation processes (i.e., photo-absorption by a molecular cation radical). 


\subsection{Intact multiply charged molecular cations}

In contrast to molecular cation radicals described above, whose thermal and photochemical reactions have been well explored, little is known about intact MMCs. However, several attractive properties are expected for intact MMCs including high electron affinity, high potential energy, high density of electronic states with various spin multiplicities and charge-dependent chemistry. The fundamentals and some applications of intact MMCs have been reviewed in detail [56-59] although these reviews have focused mainly on dications. The production of various kinds of inorganic MMCs [60] has been reported to take place by using femtosecond laser pulses [115], ion beam sputtering of solid surfaces as well as atom probe field ion microscopy $[116,117]$. The first observation of long-lived metastable diatomic inorganic tetracations, for example $\mathrm{NbHe}^{4+}$ and $\mathrm{AuNe}^{4+}$, in the gas phase was made in 1970 by Mueller et al [118]. The stabilities of inorganic tetracations have also been investigated by utilizing theoretical calculations $[119,120]$. Contrary to inorganic MMCs, the dissociation of organic MMCs is expected to occur readily partly because of the presence of $\mathrm{C}-\mathrm{H}$ bonds. The liberation of hydrogen atoms by tunneling as well as ejection of protons by Coulomb explosion is expected to take place easily in organic MMCs. In Figure 5 are shown organic molecular tetracations that have been produced by several methods such as EI [121, 122], collision with high energy projectiles [123] and femtosecond laser ionization [42, 55, 124, 125]. The organic molecular tetracation of ovalene $\left(\mathrm{C}_{32} \mathrm{H}_{14}\right)$, a large polycyclic aromatic hydrocarbon that has a structure containing 10 fused benzene rings that enable delocalization of multiple charges, was detected for the first time using EI (80 eV) [121]. In 1985, organic molecular tetracations of six aromatic hydrocarbons were generated by using EI $(70 \mathrm{eV})$, but the yields of these species were small relative to those of the corresponding molecular cation 
radicals [122]. (eg., $5 \times 10^{-8}$ (anthracene, $\mathrm{C}_{14} \mathrm{H}_{10}$ ), $1 \times 10^{-7}$ (tetracene, $\mathrm{C}_{18} \mathrm{H}_{12}$ ), $1 \times 10^{-6}$ (benz $[a]$ anthracene, $\mathrm{C}_{18} \mathrm{H}_{12}$ ), $6 \times 10^{-7}$ (chrysene, $\mathrm{C}_{18} \mathrm{H}_{12}$ ), $3 \times 10^{-8}$ (triphenylene, $\mathrm{C}_{18} \mathrm{H}_{12}$ ), $2 \times 10^{-8}$ (pyrene, $\left.\mathrm{C}_{16} \mathrm{H}_{10}\right)$ ).

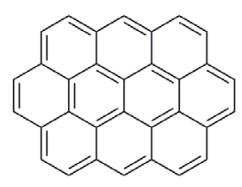

Electron ionization, $80 \mathrm{cV}$ Bursey et al. (1970)
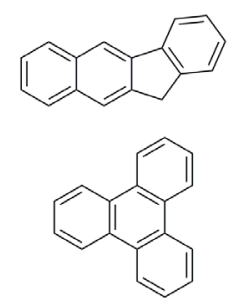

fs-laser, $1.4 \mu \mathrm{m}$ Yatsuhashi et al. (2010)
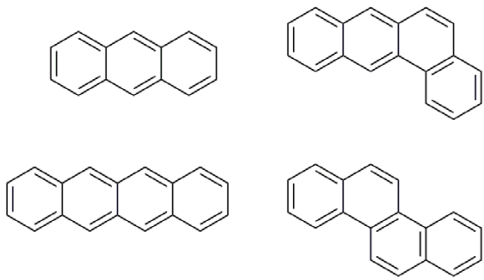

Electron ionization, $70 \mathrm{eV}$ Kingston et al. (1985)
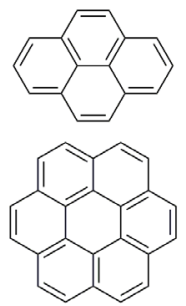

$\mathrm{Xe}^{20+}, 300 \mathrm{keV}$

Lawicki et al. (2011)

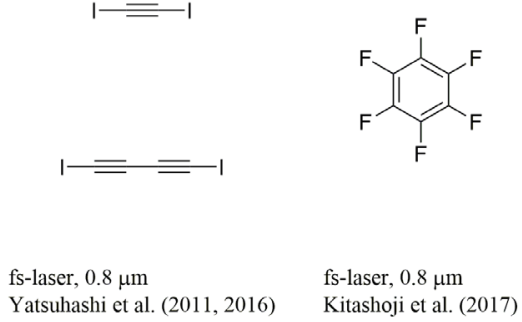

Fig. 5. Organic molecular tetracations formed by electron ionization, collision with high energy projectiles and femtosecond laser irradiation.

The relative yields of intact MMCs produced by femtosecond laser pulses are significantly high compared with that by electron ionization. Ledingham et al. detected triply charged benzene cation $\left(790 \mathrm{~nm}, 50 \mathrm{fs}, 2 \times 10^{15} \mathrm{~W} \mathrm{~cm}^{-2}\right.$ [126].) However, a lot of dissociation channels still exist for large aromatic hydrocarbons by using the fundamental wavelengths $(0.8 \mu \mathrm{m})$ of a Ti:Sapphire laser $[124,127]$. The mass spectrum is dominated by hydrocarbon-loss fragment ions and the relative abundance of intact MMC is very small. It is emphasized that the production of intact MMCs is dependent on the ionization wavelength and pulse duration of intense femtosecond lasers. The suppression of fragmentation and a dramatic increase of triply charged molecular cation yields was 
achieved by using wavelengths that are longer than $0.8 \mu \mathrm{m}$. As explained in 2.1 , this is partly due to the fact that the photo-absorption and thus accumulation of vibrational energies in molecular cation radical and/or MMCs followed by fragmentation is avoided $[94,124]$. For example, this method was used to generate trication radicals of decatetraene $\left(\mathrm{C}_{10} \mathrm{H}_{14} ; 1450 \mathrm{~nm}, 40 \mathrm{fs}, 10^{14} \mathrm{~W} \mathrm{~cm}^{-2}\right)$ [90], anthracene $\left(\mathrm{C}_{14} \mathrm{H}_{10} ; 1.4 \mu \mathrm{m}, 130 \mathrm{fs}, 5 \times 10^{13}\right.$ $\left.\mathrm{W} \mathrm{cm}{ }^{-2}\right)$ and naphthalene $\left(\mathrm{C}_{10} \mathrm{H}_{8} ; 1.4 \mu \mathrm{m}, 130 \mathrm{fs}, 1.5 \times 10^{14} \mathrm{~W} \mathrm{~cm}^{-2}\right.$ [128]). The relative yield of triphenylene $\left(\mathrm{C}_{18} \mathrm{H}_{12}\right)$ tetracation formation by using $1.4 \mu \mathrm{m}$ femtosecond laser pulses was 14,000 times larger than that obtained by employing EI [124], leaving no doubt that femtosecond laser ionization has a potential to produce intact MMCs in abundance. For the case of aromatic hydrocarbons, the use of collision with high energy projectiles $\left(\mathrm{Xe}^{20+}, 360 \mathrm{keV}\right)$ also enables improvements in MMCs production yields [123, 129, 130].

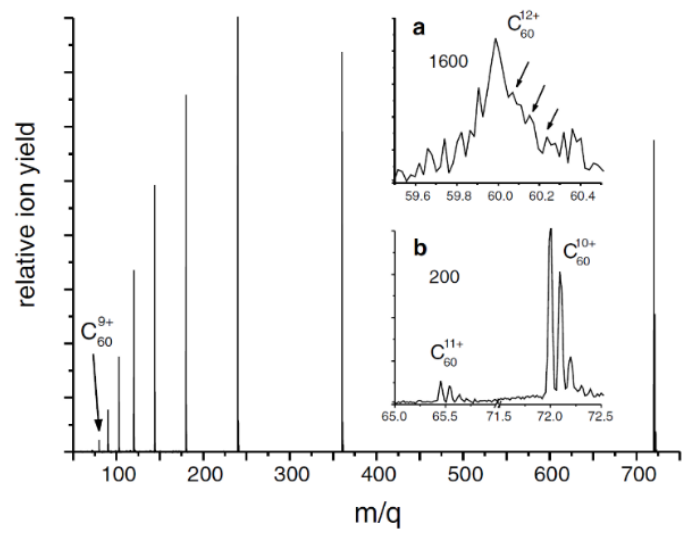

Fig. 6. Mass spectra of $\mathrm{C}_{60}{ }^{z+}(z \leqq 12)$ measured by using fs-pulses (1800 nm, $70 \mathrm{fs}, 10^{15}$ $\mathrm{W} \mathrm{cm}{ }^{-2}$ ). The insets are magnifications of three highest charged cations. Reprinted with permission from Ref. [131]. Copyright 2003 American Physical Society.

Bhardwaj et al. described an MMC with the highest charge number obtained to date is 12 using fullerene (Fig. 6) [131]. Recently, detection of the pentacation radical of methane was reported by using the irradiation of a small methane cluster with 
femtosecond laser pulses $\left(624 \mathrm{~nm}, 100 \mathrm{fs}, 8 \times 10^{14} \mathrm{~W} \mathrm{~cm}^{-2}\right)$ [132]. However, this is unlikely because the multiply charged ion arising by removal five out of eight valence electrons should be highly prone to dissociation [133].

The potential energy surfaces of MMCs are determined by the balance between repulsive Coulombic forces and chemical bonding. When the charge number is large, instantaneous fragmentation of MMCs is expected to occur especially for small molecules. The only exception found to date is the MMC of diiodoacetylene $[42,55,125]$. It is worthy to note that the smallest organic tetracation $\mathrm{ICCI}^{4+}$ was formed using $0.8 \mu \mathrm{m}$ pulses $[42,55,125]$, while $\mathrm{ICCI}^{3+}$ was the highest charge number state formed by using collision with $\mathrm{Ar}^{8+}(3 \mathrm{keV})$ [134]. The unusual stability of $\mathrm{ICCI}^{4+}$ is explained in terms of charge localization on the terminal iodine atoms, which leads to minimum Coulomb repulsion between adjacent atoms as well as maximum charge-induced attractive dipole interactions between iodine and carbon [55]. Recently, we successfully produced the smallest aromatic tetracation, $\mathrm{C}_{6} \mathrm{~F}_{6}{ }^{4+}$ by using femtosecond laser pulses [135]. As a dramatic increase in the charge numbers observed takes place, intact MMCs will become worthwhile subjects to investigate, especially in terms of their charge-dependent spectroscopic properties. In addition, exploring chemical reactions as well as theoretical calculations of intact MMCs are great challenges of physics and chemistry [136].

\section{Coulomb explosion}

MMCs dissociate into atomic and/or molecular fragment ions with high kinetic energy by Coulomb explosion. In the history of Coulomb explosion studies, in the 1990's various kind of small MMCs were extensively investigated. Codling and Frasinski reviewed the results of studies with $\mathrm{N}_{2}, \mathrm{HCl}, \mathrm{H}_{2}, \mathrm{D}_{2}, \mathrm{I}_{2}, \mathrm{CO}, \mathrm{N}_{2} \mathrm{O}, \mathrm{CO}_{2}, \mathrm{C}_{2} \mathrm{H}_{2}$, and $\mathrm{C}_{2} \mathrm{H}_{4}$ 
in 1994 [20]. Reports on femtosecond ionization of complex molecules increased in 1998 and ionization and dissociation of large molecules by femtosecond pulses was reviewed by Levis and DeWitt in 1999 [137]. We summarized the vigorous discussions about the ionization of organic molecules by using the fundamental wavelength $(0.8 \mu \mathrm{m})$ of a Ti:Sapphire laser during 1997-1999, in addition to the Coulomb explosion of benzene and fullerene in 2000 [18]. Reviews discussing the ionization of fullerene were published by Hertel et al. in 2005 [138] and by Johansson and Campbell in 2013 [139].

In the past two decades, Coulomb explosion of various kinds of molecules by using intense femtosecond laser fields has been intensively explored. There is no doubt that simple two- and few-atomic molecules are very important substrates to test newly developed theories and experimental methods [140]. Despite this, we have listed here only selected studies performed from 2004 to data on Coulomb explosion of isolated large molecules initiated by femtosecond laser pulses. The family of molecules include aliphatic and [52, 125, 141-159] aromatic hydrocarbons [92, 160-162], halogenated molecules [33, 42, 163-176] and alcohols [49, 51, 53, 155, 177-182]. In addition, examples of organometallics [183, 184], organic acids [185], amines [186], molecules containing silicon [187, 188], nitriles [189], ketones [96, 155], heterocycles [95], nitroaromatic compounds [190], and $\mathrm{SF}_{6}$ and its anion [191, 192] are also investigated.

\subsection{Observation of Coulomb explosion}

Observations of ions ejected during Coulomb explosion have been made by using mass-selective instruments such as a TOF-MS where they are detected by using a microchannel plate (MCP). A linear relationship between the time of flight and the initial momentum of a fragment ion along the ion flight axis exists when a Wiley-McLaren 
configuration is employed [193]. Thus, a simple Wiley-McLaren TOF-MS equipped with a conventional MCP can be used to determine kinetic energies and momenta. The typical feature of an ion generated by Coulomb explosion is the presence of split peaks in the TOF spectrum. In Figure 7 are shown the doublet and/or multiplet peaks observed for $\mathrm{H}^{+}$, $\mathrm{H}_{2}{ }^{+}$and $\mathrm{H}_{3}{ }^{+}$ejected by Coulomb explosion of MMC of ethane (800 nm, $100 \mathrm{fs}, 1 \times 10^{14}$ $\mathrm{W} \mathrm{cm}^{-2}$ ) [145]. These features are a consequence of the fact that ions with certain kinetic energies are emitted along both the forward and backward direction relative to the ion flight axis, which is determined by the applied static electric field. These respective ions are detected either at earlier or later times in the TOF spectrum. The peak associated with an ion not having kinetic energy would be found in the center of multiplet.
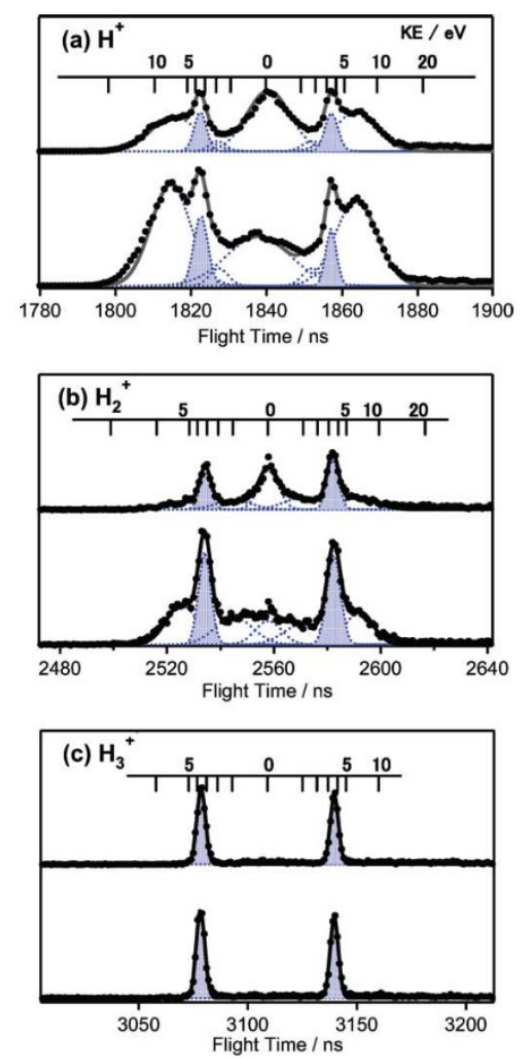

Fig. 7. Time-of-flight spectra of (a) $\mathrm{H}^{+}$, (b) $\mathrm{H}_{2}{ }^{+}$, and (c) $\mathrm{H}_{3}{ }^{+}$ejected from the MMC of ethane. Upper and lower traces are those measured with laser polarization orthogonal and 
parallel with respect to the ion flight axis. The kinetic energy scale is also presented for both forward and backward emitted ions. The components originated from ethane dication are shadowed. Adapted with permission from Ref. [145]. Copyright 2011 AIP Publishing LLC.

The kinetic energy, $E_{k}$ (in J unit), and momentum $p$ (in $\mathrm{kg} \mathrm{m} \mathrm{s}^{-1}$ unit), of an ion are calculated using equations 2 and 3, respectively.

$$
\begin{aligned}
& E_{k}=\frac{(z e)^{2}}{2 m} F^{2}\left(t_{1}-t_{0}\right)^{2} \\
& p=z e F\left(t_{1}-t_{0}\right)
\end{aligned}
$$

, where $z, e, m, F, t_{1}$, and $t_{0}$ are the charge number of the ion, elemental charge (in $\mathrm{C}$ unit), mass of the ion (in $\mathrm{kg}$ unit), electric field for extraction (in $\mathrm{V} \mathrm{m}^{-1}$ or $\mathrm{N} \mathrm{C}^{-1}$ unit), flight time of the ion (in s unit) emitted forward to the detector and the calculated flight time of ion with zero kinetic energy (momentum), respectively. By taking into account momentum conservation, the counterpart of ion in the Coulomb explosion process can be identified, especially for diatomic molecules. The momentum spectra of nitrogen ions generated by two-body Coulomb explosion of $\mathrm{N}_{2}\left(795 \mathrm{~nm}, 100 \mathrm{fs}, 3.5 \times 10^{15} \mathrm{~W} \mathrm{~cm}^{-2}\right)$ is shown in Fig. 8 [194]. By adhering to the momentum conservation law, it is possible to identify the different Coulomb explosion pathways occurring, the channels for which clearly resolved in Fig. 8, where $(p, q)$ denotes the fragment ions formed by the Coulomb explosion of $\mathrm{N}_{2}^{(p+q)+} \rightarrow \mathrm{N}^{p+}+\mathrm{N}^{q+}$. Based on the charge number and kinetic energy (or momentum) of ions participating in the Coulomb explosion, the bond distance of diatomic molecules at the moment of Coulomb explosion can be calculated. It is noted that momentum matching can also be applied to treat two-body Coulomb explosions of molecular dimers (see 4.2.2). 


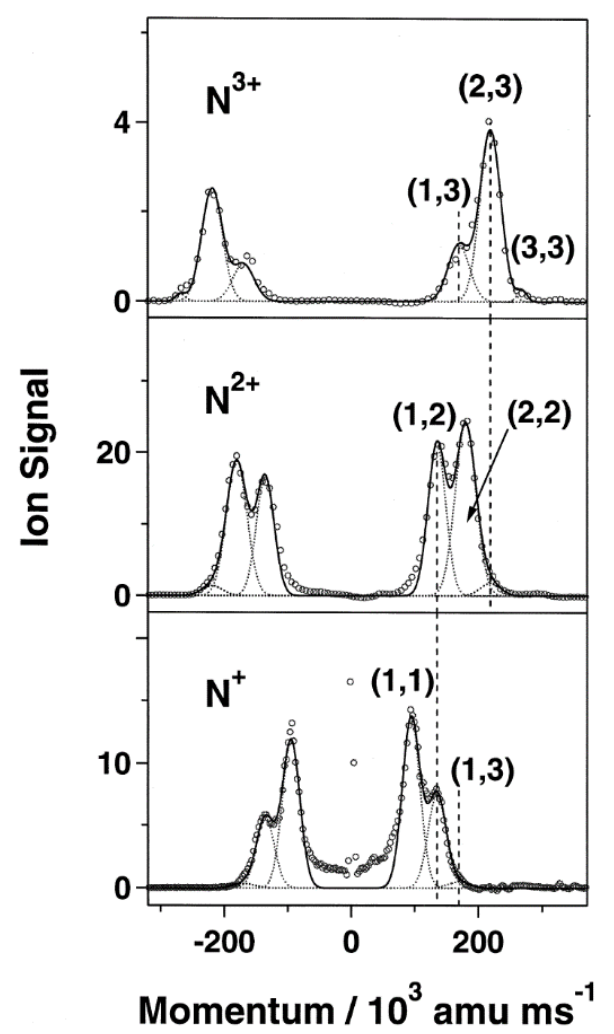

Fig. 8. The momentum spectra of $\mathrm{N}^{+}, \mathrm{N}^{2+}$, and $\mathrm{N}^{3+}$ ejected from $\mathrm{N}_{2}$. The assignment of explosion channels, $\mathrm{N}_{2}^{(p+q)+} \rightarrow \mathrm{N}^{p+}+\mathrm{N}^{q+}$, are denoted as $(p, q)$. Reprinted with permission from Ref. [194]. Copyright 1998 Elsevier.

Another important feature of Coulomb explosion initiated by using an intense linearly polarized laser is anisotropic ejection of ions with respect to the laser polarization direction. Supposing that linearly polarized laser pulse is used for ionization, the probability of tunnel ionization of molecules depends on how they are aligned with respect to the polarization direction of laser pulse [40]. Because the ensuing multiple ionization processes will be regulated by the initial ionization step, anisotropic emission of ions from MMCs with respect to the laser polarization direction is observed. 


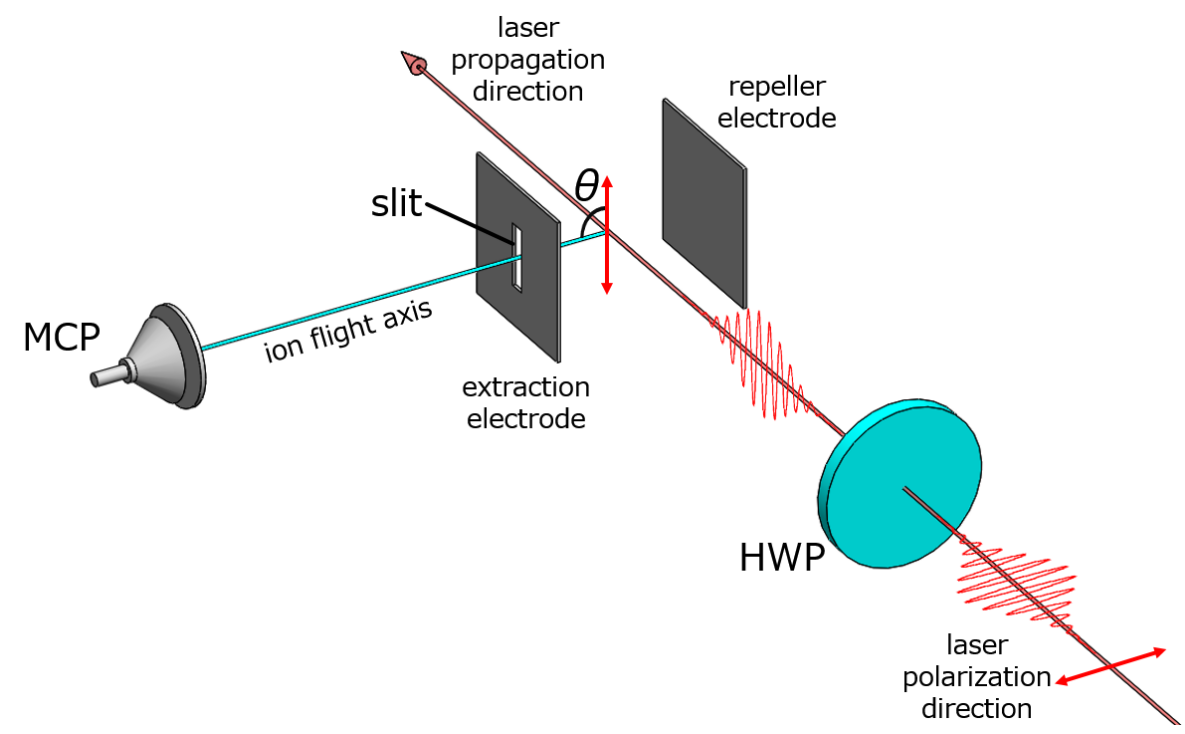

Fig. 9. Schematic of the configuration between the ion flight axis and the laser polarization direction. The angle $\theta$ measured with respect to the laser polarization direction from the ion flight axis to the detector. HWP denotes a half-wave plate. Adapted with permission from Ref. [125]. Copyright 2016 Elsevier.

Figure 9 contains an illustration of the configuration of the ion source in a TOF-MS, consisting of a repeller electrode, an extraction electrode, an einzel lens (not shown), electrostatic deflectors (not shown) and a MCP. A narrow slit is located on the extraction electrode perpendicular to the laser propagation direction in order to collect ions that are generated at the most tightly focused point of the laser beam (achieving ion collection from an axially symmetric parallel beam geometry). The direction of laser polarization (linear) with respect to the ion flight axis is changed by using a half-wave plate. When the direction of laser polarization is parallel $(\theta=0,180$ degree $)$ or perpendicular $(\theta=90,270$ degree $)$ to the ion flight axis, the conditions are referred to as "parallel" or "orthogonal," respectively. In the cases of diatomic molecules, emission of ions is greatly diminished under the orthogonal condition. Thus, the extinction of ions, 
that is, the ratio of the ion intensity measured under an orthogonal condition to that measured under a parallel condition is nearly zero. In the cases of large and complex molecules, TOF spectra can provide information about only kinetic energy (momentum) and ion yields. In spite of this, by considering the anisotropy (extinction) of ion emissions, these data give important information about the molecular structure and Coulomb explosion processes.

For example, the angular distribution and the kinetic energy spectra of carbon and oxygen ions ejected from the $\mathrm{MMC}$ of $\mathrm{Cr}(\mathrm{CO})_{6}$ are displayed in Fig. 10. It is obvious that the extinction of oxygen ion is smaller than 1 . In contrast, extinctions of carbon ions are larger than 1, and are largely seen at higher kinetic energy. Supposing that the most preferable ionization axis is along a line inclined $45^{\circ}$ relative to $\mathrm{CO}-\mathrm{Cr}-\mathrm{CO}$ axis in a $\mathrm{Cr}(\mathrm{CO})_{4}$ plane, and four configurations of molecules with respect to laser polarization direction and ion flight axis are considered as shown in Fig. 11, we can evaluate the extinctions of oxygen and carbon ions to be 0.75 and 1.5 , respectively. Although the above simplified considerations overestimate the extinction of oxygen and underestimate the extinction of carbon ions, the characteristic extinction values can be qualitatively explained [183].
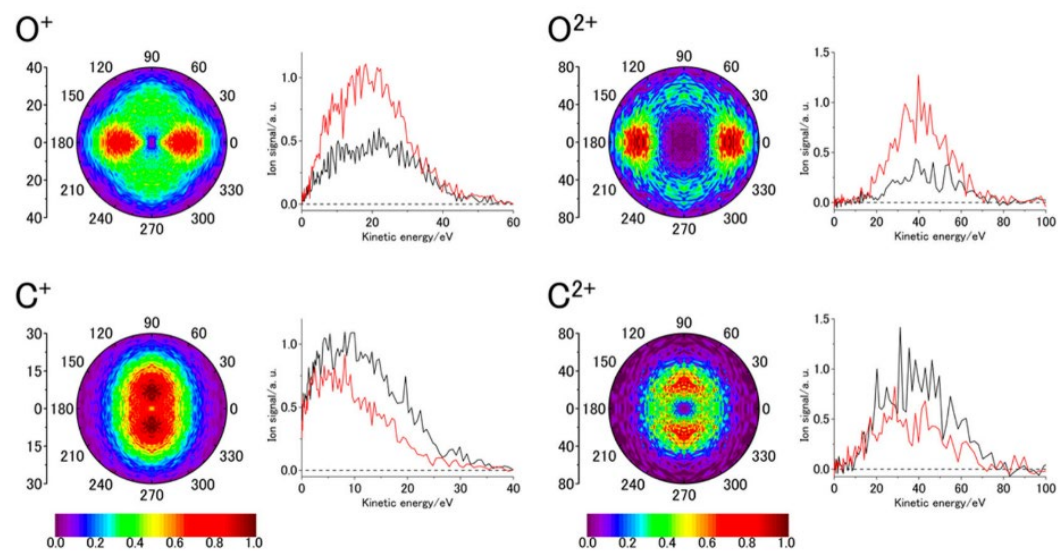

Fig. 10. Angular distribution and kinetic energy spectra of $\mathrm{O}^{+}, \mathrm{O}^{2+}, \mathrm{C}^{+}$, and $\mathrm{C}^{2+}$ ejected 
from $\mathrm{Cr}(\mathrm{CO})_{6}$ at $1.3 \times 10^{14} \mathrm{~W} \mathrm{~cm}^{-2}$. Angle in the polar plot indicates the relative angle between the polarization plane of the laser fields and the ion flight axis to the detector. The radius of the polar plot indicates kinetic energy in eV units. The red and black lines in the kinetic energy spectra give data obtained under parallel ( 0 and 180 degrees) and orthogonal (90 and 270 degrees) conditions, respectively. Reprinted with permission from Ref. [183]. Copyright 2016 American Chemical Society.

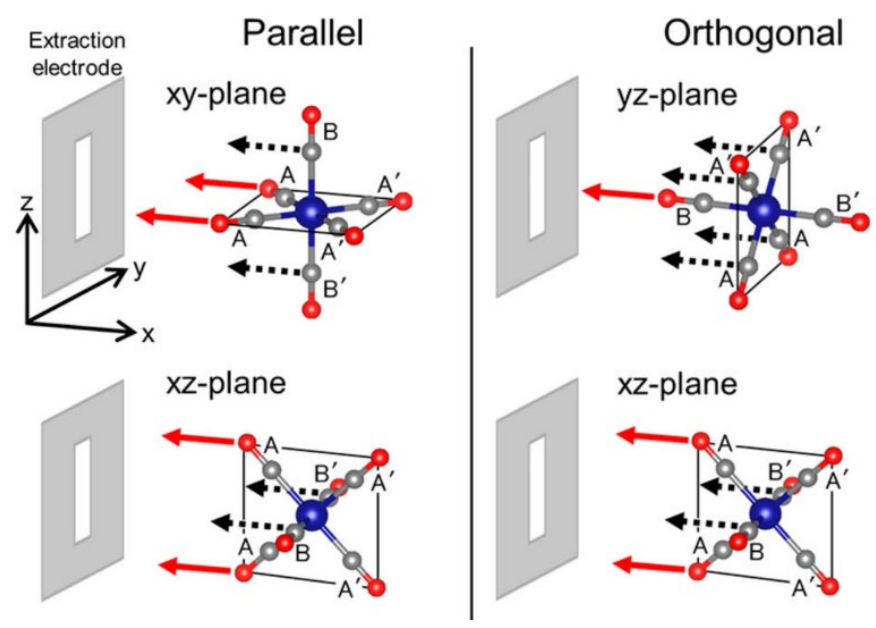

Fig. 11. Schematics of the tunnel ionization of $\mathrm{Cr}(\mathrm{CO})_{6}$ when the laser electric field is parallel to a line inclined $45^{\circ}$ relative to $\mathrm{CO}-\mathrm{Cr}-\mathrm{CO}$ axis in a $\mathrm{Cr}(\mathrm{CO})_{4}$ plane under the parallel (left) and orthogonal (right) conditions. The ion flight axis (x), the extraction electrode with a slit, and laser propagation direction (y) are also shown. The z-axis is parallel to the plane of the extraction electrode that is perpendicular to the $\mathrm{x}$ - and $\mathrm{y}$-axes. Arrows indicate the emission of oxygen (red solid arrows) and carbon ions (black dotted arrows) that contribute the kinetic energy spectrum and angular distribution. Adapted with permission from Ref. [183]. Copyright 2016 American Chemical Society.

Even though qualitative interpretations of ion emissions even for large molecules 
can be made to some extent by considering preferable ionization axes and molecular symmetry, the actual structure during the Coulomb explosion cannot be obtained because correlations between ions are not known. Investigations of the structure deformation at the moment of Coulomb explosion by using two-dimensional momentum measurements, namely mass-resolved two-dimensional momentum imaging, have been carried out by Hishikawa et al. [194-197]. The covariance mapping technique [198-200] as well as ionion coincidence measurements [201] have been utilized to determine exact correlations between fragment ions (i.e., fragmentation channels) for small molecules. Recently, covariance mapping has been increasingly used to investigate the dynamics of inner shell electron ionization brought about by using intense XFELs. Coulomb explosion imaging, i.e., measurement of three-dimensional momenta of ions, has become an important technique in physics and chemistry since the invention of velocity map imaging technique [202].

\subsection{Covariance mapping}

Coulomb explosion can be identified by using a TOF-MS. Because this instrument basically gives one-dimensional arrival time information, it cannot be employed to clarify relationships between ions. The covariance mapping technique, which extends a one-dimensional TOF spectrum into a two-dimensional correlation map, can be utilized to determine correlations between ions from TOF data [198-200]. Coincidence measurements (single coincident event per laser shot) described later cannot be utilized to correlate ions if more than one neutral molecule is ionized. In contrast to coincidence measurements, covariance mapping can be utilized for the multiple coincident events occurring per laser shot. Thus, this method can be utilized 
even using pressures in the $10^{-7} \mathrm{~Pa}$ range (ca. $4 \times 10^{-14} \mathrm{~mol} \mathrm{dm}^{-3}$ at $300 \mathrm{~K}$ ), which enable generation of sufficient amounts of ions to gain reliable signals using a conventional oscilloscope. Covariance mapping measures the fluctuations of ion signals in a TOF spectrum on a shot-to-shot basis. Correlations of ion intensity fluctuations occur only when fragment ions arise from the same fragmentation channel. The covariance coefficient $C(X, Y)$ between the TOFs of $X$ and $Y$ is defined by equation 4 .

$C(X, Y)=\langle(X-\langle X\rangle)(Y-\langle Y\rangle)\rangle=\langle X Y\rangle-\langle X\rangle\langle Y\rangle$

, where the mean values \langle\rangle are obtained over $N$ laser shots. Here, we evaluate the covariance coefficient $C\left(X\left(t_{1}\right), Y\left(t_{2}\right)\right)$, representing covariance between ions detected at $t_{1}$ and at $t_{2}$ is therefore expressed in equation 5.

$C\left(X\left(t_{1}\right), Y\left(t_{2}\right)\right)=\left\langle X_{i}\left(t_{1}\right) Y_{i}\left(t_{2}\right)\right\rangle-\left\langle X_{i}\left(t_{1}\right)\right\rangle\left\langle Y_{i}\left(t_{2}\right)\right\rangle$

$=\frac{1}{N} \sum_{i=1}^{N} X_{i}\left(t_{1}\right) Y_{i}\left(t_{2}\right)-\left[\frac{1}{N} \sum_{i=1}^{N} X_{i}\left(t_{1}\right)\right] \times\left[\frac{1}{N} \sum_{i=1}^{N} Y_{i}\left(t_{2}\right)\right]$

, where $X_{i}$ and $Y_{i}$ are number of ions measured at $i$ th laser shot. Covariance mapping has been successfully applied to treatment of few-atomic molecules [20, 39, 40, 197, 200], as well as large molecules [33, 43, 203-205] and clusters [206-209].

The most notable and straightforward success of covariance mapping was its application to two-body Coulomb explosion of diatomic molecules [210]. In Figure 12(a) is given the covariance map of $\mathrm{N}_{2}$ at a pressure of $5 \times 10^{-7} \mathrm{~Pa}$ determined using linearly polarized laser pulses $\left(800 \mathrm{~nm}, 40 \mathrm{fs}, 3 \times 10^{15} \mathrm{~W} \mathrm{~cm}^{-2}\right)$, and where the ion detection was limited by a $1 \mathrm{~mm}$ diameter hole in the electrode. In this covariance map, $X_{i}$ and $Y_{i}$ are taken from the same TOF spectrum. The strong diagonal line in the map in Fig. 12(a) corresponds to the $\left\langle X_{i}^{2}\right\rangle-\left\langle X_{i}\right\rangle^{2}$ component. The mirror symmetry about the diagonal line is attributed to the fact that $C\left(X\left(t_{1}\right), Y\left(t_{2}\right)\right)=C\left(X\left(t_{2}\right), Y\left(t_{1}\right)\right)$. The eight islands on the map correspond to correlations between the ion pairs, $C\left(\mathrm{~N}^{2+}, \mathrm{N}^{2+}\right), C\left(\mathrm{~N}^{3+}, \mathrm{N}^{2+}\right)$, 
$C\left(\mathrm{~N}^{2+}, \mathrm{N}^{3+}\right)$, and $C\left(\mathrm{~N}^{3+}, \mathrm{N}^{3+}\right)$. The origin of the respective fragment ion pairs is identified as being $\mathrm{N}_{2}{ }^{4+}, \mathrm{N}_{2}{ }^{5+}, \mathrm{N}_{2}{ }^{5+}$, and $\mathrm{N}_{2}{ }^{6+}$. In Figure $12(\mathrm{a}), \mathrm{N}^{z+} \mathrm{f}$ and $\mathrm{N}^{z+}$ b denote the $z$ th charged nitrogen atoms emitted in a forward and backward direction relative to the detector. Thus, islands corresponding to $C\left(\mathrm{~N}_{f}^{Z+}, \mathrm{N}_{f}^{Z+}\right)$ and $C\left(\mathrm{~N}_{b}^{Z+}, \mathrm{N}_{b}^{Z+}\right)$ should not exist. Owing to the conservation of momentum, the tilt angle, $\alpha$, indicated in Fig. 12(b) reflects the charge ratio of the ion pairs. The observed tilt angles well match the calculated values, which are 45 degrees $(\tan \alpha=1)$ for $C\left(\mathrm{~N}^{2+}, \mathrm{N}^{2+}\right)$ and $C\left(\mathrm{~N}^{3+}, \mathrm{N}^{3+}\right)$, and 34 degrees $(\tan \alpha=2 / 3)$ for $C\left(\mathrm{~N}^{2+}, \mathrm{N}^{3+}\right)$ and $C\left(\mathrm{~N}^{3+}, \mathrm{N}^{2+}\right)$.

(a)

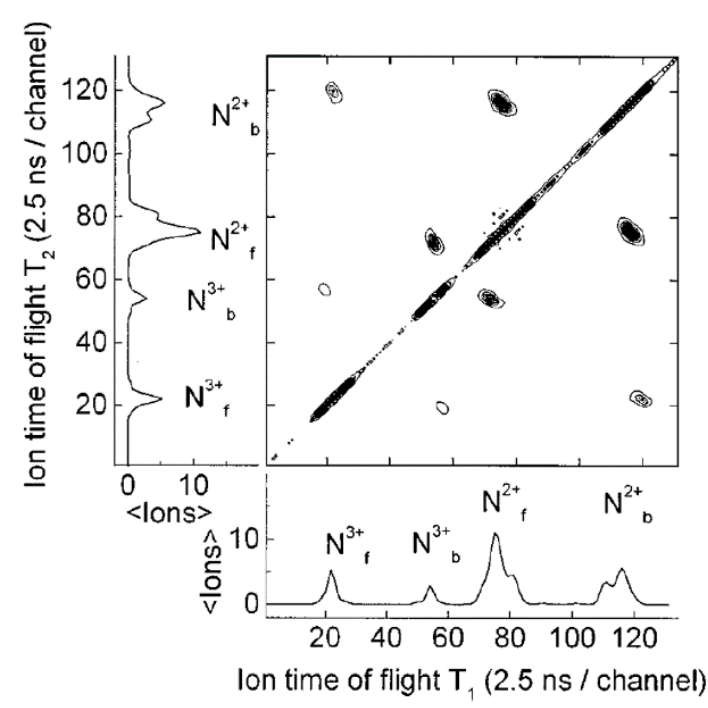

(b)

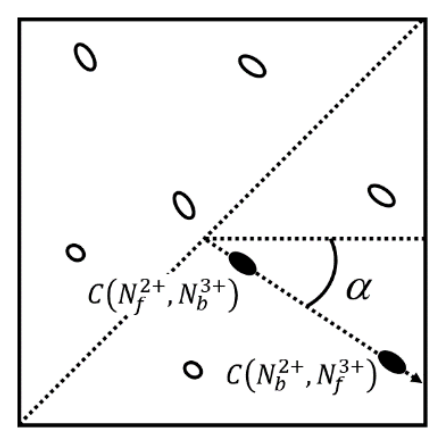

Fig. 12. (a) Covariance map of $\mathrm{N}_{2}$. The bottom and left traces represent average time-offlight spectra. Reprinted with permission from Ref. [210] . Copyright 1999 American Physical Society. (b) Schematic illustration of the tilt angle $\alpha$ for a pair of $C\left(\mathrm{~N}^{2+}, \mathrm{N}^{3+}\right)$.

Covariance mapping is now utilized in many fields of science and technology [198]. The angle-resolved covariance mapping generated by using a two-dimensional detector has proven to be a powerful tool for the determination of molecular structure 
(see 4.1.1). In recent years, Coulomb explosions initiated by ionization of inner shell electrons have been explored by using intense XFELs [211, 212]. Utilization of the combination of covariance mapping and XFELs is an ideal because of the high intensity but low repetition rate inherent in XFEL experiments.

\subsection{Velocity map imaging}

The advent of time and position sensitive detectors, for example a MCP equipped with multi-hit delay-line anodes or with a phosphor screen backed by an intensified charge coupled device, enables recording of both the hit position and TOF information of ions. In 1997, Eppink and Parker invented the velocity map imaging (VMI) technique [202]. VMI is a type of TOF mass spectrometry, in which ions of the same mass and initial velocity are guided to the same position on a two-dimensional detector by optimizing the electric fields in the ion source. VMI ion optics consist of a repeller, an extractor and a ground electrode as is employed in a Wiley-McLaren setup, but in this case extractor and ground electrodes have open holes. In Figure 13 are displayed simulated ion trajectories in a VMI instrument. Although the origin position of the ions differs by $3 \mathrm{~mm}$, ions with the same initial ejection angle impact the same position on the focal plane. The trajectories indicated by 1, 2, and 3 in Fig. 13 correspond to ejection angles of 0 and 180 degree (in $\mathrm{x}$ direction), 45 and 135 degrees (in y direction), and 90 degrees (in y direction), respectively. Another benefit of VMI is that the shape of the ion trajectory is independent of the charge of ion. 


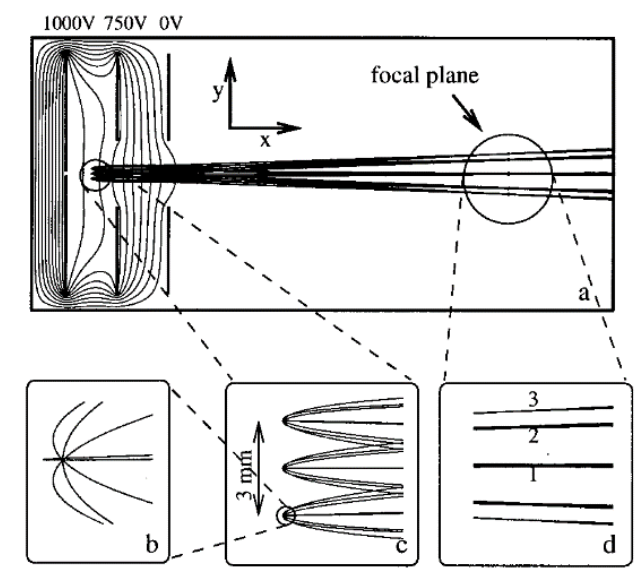

Fig. 13. Simulated trajectories of ions formed at different initial positions and with different initial ejection angles in velocity map instruments. Reprinted with permission from Ref. [202]. Copyright 1997 AIP Publishing LLC.

A schematic of the VMI setup for photodissociation experiments is shown in Fig. 14. In this setup, laser 1 initiates photodissociation and laser 2 ionizes the resulting fragments. The polarization of the ionizing laser 2 is parallel to the detector plane. The ions generated by Coulomb explosion expand on a Newton sphere defined by the conservation of momentum, i.e., ions of the same initial velocity are on the same surface of the sphere under VMI conditions. Because a linearly polarized laser pulse is used, the symmetry of the velocity distribution is cylindrical around the laser polarization direction denoted by $\vec{E}$. An expanding Newton sphere is accelerated along the ion flight axis and, thus, Newton spheres for different ions are mass-separated. Newton spheres are projected on the two-dimensional detector and ion-images $D(z, x)$ are recorded. Because the momentum distribution is anisotropic along with $\vec{E}, D(z, x)$ becomes two opposing crescent shapes. The velocities of ions arise from 1) the impact position on the detector, where the kinetic energy is proportional to the square of the ring radius, 2) the distance between the ionization zone and the detector, and 3) the arrival time of the ions. Based on 
the knowledge of the mass and charge of ions, the corresponding linear momenta of ions can be reconstructed. The ion image $D(z, x)$ is mathematically transformed (Abel inversion) to obtain a velocity map $F(z, r)$, where $r$ is the coordinate perpendicular to $z$ axis. Furthermore, the full three-dimensional momentum vectors of ions in a singlemolecule basis are obtained if we detect ions ejected from a molecule in coincidence.

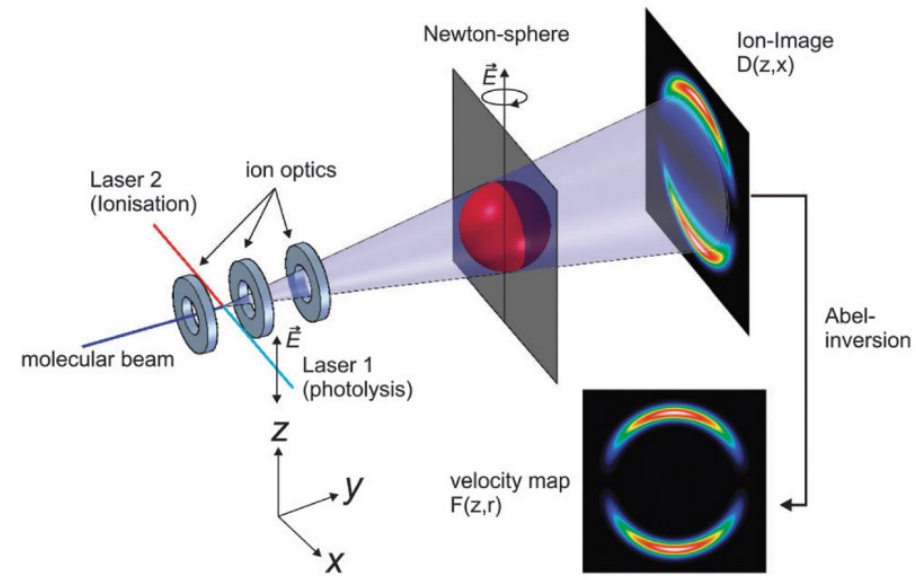

Fig. 14. Schematic setup of a velocity map imaging experiment showing the relation between the Newton sphere, ion image, and velocity map image. Reprinted with permission from Ref. [213]. Copyright 2014 Royal Society of Chemistry.

The details of VMI have been reviewed before [214-216]. Below, we only briefly discuss recent studies of VMI. Techniques for recovering of three-dimensional momentum from a two-dimensional ion image without using mathematical procedures, referred to as slice imaging, have been developed. Slice imaging is achieved by slicing the middle of a Newton sphere. Several approaches have been proposed for this purpose including optical, Doppler, DC and pulsed field slicing [216]. Imaging only the central part of a Newton sphere by utilizing a fast gating detector results in equivalent data quality as those obtained by employing Abel inversion of a conventional ion image [215]. 
Nowadays, VMI is used in a wide range of research fields such as photoelectron spectroscopy [217], photodissociation dynamics investigations [218] and attosecond pulse characterization [219]. CEIs with VMI have also been used to explore electronic structural changes [220] and chemical reaction dynamics [221, 222]. Hydrogen migration in cationic and/or dicationic species is one of the most interesting chemical reactions occurring in intense laser fields. Yamanouchi and co-workers have studied in detail hydrogen migration taking place in ions derived from acetonitrile $[48,50]$, methanol [49, $51,53,179-181]$, allene $[52,149]$, butadiene $[150,151]$, and methylacetylene [153].

Another substance that has attracted great attention in the field of intense laser chemistry is acetylene. The isomerization reaction of acetylene $(\mathrm{HC} \equiv \mathrm{CH})$ to vinylidene $\left(\mathrm{CCH}_{2}\right)$ has been investigated by using pump and CEI probe methods. A pump pulse ionizes acetylene to form its cation radical or dication which undergo isomerization by hydrogen migration, and a probe pulse is used to excite transient states to the Coulombic repulsive states $\left(\mathrm{C}_{2} \mathrm{H}_{2}{ }^{3+}\right)$ which dissociate into atomic and molecular fragment ions by Coulomb explosion. The time evolution of three-dimensional momenta of fragment ions are determined by using coincidence VMI and by varying the time delay between the pump and probe pulses [156, 157, 223, 224].

Some applications of coincidence VMI are introduced in section 4.1.2. It should be mentioned that another powerful tool for carrying out three-dimensional momentum measurements for the imaging molecular structures is cold target recoil-ion momentum spectroscopy (COLTRIMS) and/or reaction microscopy [225, 226]. The applications of COLTRIMS method will be discussed in sections 4.1.3 and 4.2.1.

\section{Coulomb explosion of molecules}




\subsection{Isolated Molecules}

Ions generated by Coulomb explosion of molecules have specific and characteristic momenta. Therefore, the reconstruction of molecular structure is possible by the measurements of three-dimensional momenta of the ions in covariance and/or coincidence. In the case of diatomic molecules, the correlation of momenta of two fragment ions gives the internuclear distance at the moment of Coulomb explosion. The deformation of the molecular structures of tri- and multi-atomic molecules in intense laser fields has been a central topic of several studies [194, 195, 227]. At the current time, we are able to investigate the structure of large and complex molecules, as well as geometric isomers, positional isomers and enantiomers. In this section, the recent progresses about the discrimination of molecular structure are reviewed.

\subsubsection{Complex molecules}

Recently, Slater et al. have shown that covariance measurement of CEI can be utilized to visualize the structure of the specific but relatively large molecule, 3,5dibromo-3',5'-difluoro-4'-cyanobiphenyl (BP, $\mathrm{C}_{13} \mathrm{Br}_{2} \mathrm{~F}_{2} \mathrm{H}_{5} \mathrm{~N}$ ), with the aid of both supersonic expansion cooling and adiabatic molecular alignment [43, 203, 204]. BP is first adiabatically aligned in the laboratory frame by using a nanosecond laser pulse (1064 $\mathrm{nm}, 10 \mathrm{~ns}, 20 \mathrm{~Hz}, 8 \times 10^{11} \mathrm{~W} \mathrm{~cm}^{-2}$ ) and then irradiated using an intense femtosecond laser pulse $\left(800 \mathrm{~nm}, 30 \mathrm{fs}, 3 \times 10^{14} \mathrm{~W} \mathrm{~cm}^{-2}\right)$. The most polarizable axis of BP, i.e., $C_{2}$ symmetry axis, which is along the $\mathrm{C}-\mathrm{C}$ bond between the two phenyl rings, is one-dimensionally (head-to-tail or tail-to-head) aligned along the polarization direction of nanosecond laser. In this case, the relative alignment of BP with respect to the detector plane can be fixed, but molecules are allowed to rotate around a $C_{2}$ symmetry axis. 
In Figure 15(a) are shown the laboratory-frame image of $\mathrm{H}^{+}$(left) and the covariance image of $\mathrm{H}^{+}$with respect to $\mathrm{N}^{+}$(right) taken by aligning BP parallel to the detector plane. The laboratory-frame image of $\mathrm{H}^{+}$is symmetric with respect to the horizontal and vertical axes because BP, which contains four hydrogen atoms around the waist and one hydrogen atom on its tail, freely rotates around its $C_{2}$ symmetry axis. In contrast, strong asymmetry with respect to the horizontal axis is present in the covariance image because the covariance image represents the two-vector velocity correlation between $\mathrm{H}^{+}$and $\mathrm{N}^{+}$. The asymmetry is representative of the asymmetric location of five hydrogen atoms marked by red circles with respect to the nitrogen atom marked by a blue circle in Fig. 15(a). In contrast to the images taken by aligning BP parallel to the detector plane, less information (one spot in the middle of image) comes from the covariance image in the perpendicular alignment (aligned normal to the detector plane, not shown in this review, please refer Fig. 7 in Ref. [43]) because $\mathrm{H}$ and $\mathrm{N}$ are collinearly ejected toward the detector.

(a)

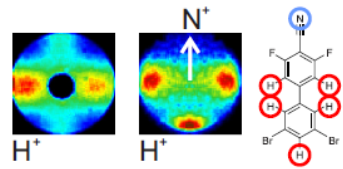

(b)
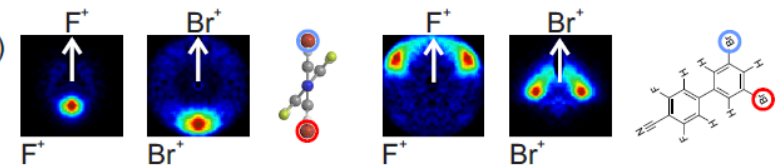

(c)
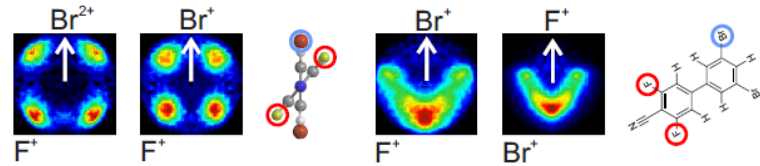

Fig. 15. (a) (left) Laboratory-frame image of $\mathrm{H}^{+}$and (right) covariance image of $\mathrm{H}^{+}$with respect to $\mathrm{N}^{+}$. (b) and (c) Covariance image of $\mathrm{A}^{+}$with respect to $\mathrm{B}^{z+}$. $\mathrm{A}$ and $\mathrm{B}$ are indicated below and above the image, respectively. BP is aligned parallel (a, right panels of $b$ and $c$ ) and perpendicular (left panels of $b$ and $c$ ) to the detector plane. Reprinted 
with permission from Ref. [43]. Copyright 2015 American Physical Society.

The so-called autocovariance images are shown in Fig. 15(b) for different alignments of BP. The images taken in the perpendicular alignment (Fig. 15(b), left panel) represent that one fluorine (bromine) ion marked by a blue circle is ejected upward as indicated by the white arrow in the image, and the other fluorine (bromine) ion marked by a red circle is ejected in the opposite direction (downward). This is a consequence of the fact that the two fluorine (bromine) atoms are located at meta positions of the same phenyl ring. In the parallel alignment (Fig. 15(b), right panel), the effect of free rotation around $C_{2}$ symmetry axis is reflected in the presence of two peaks in the autocovariance images.

In contrast to the autocovariance images (Fig. 15(b)) and the covariance image of $\mathrm{H}^{+}$(Fig.15 (a) right), the covariance images of fluorine ion with respect to bromine ion in the perpendicular alignment indicate the dihedral angle between the two phenyl rings (Fig. 15(c), left panel). A pair of peaks that represent the relative locations of two fluorine atoms are indicated by the red circles in the left panel of Fig. 15(c). The clearly separated four covariance peaks, arising from rotation of molecules around $C_{2}$ symmetry axis, are comprised of two pairs of peaks oriented diagonally. An inverse Abel transformation can be applied to improve the accuracy of covariance image by removing randomness in the alignment of the planes of the two phenyl rings. After the processing, the recoil angles of $\mathrm{F}^{+}$and $\mathrm{Br}^{+}$relative to the $\mathrm{N}^{+}$were precisely determined to be $61.7 \pm 0.2$ and $127.1 \pm 0.3$ degree, respectively [43]. The covariance images of $\mathrm{F}^{+}$with respect to $\mathrm{Br}^{+}$and $\mathrm{Br}^{+}$with respect to $\mathrm{F}^{+}$in parallel alignments are shown in the right panel of Fig. 15(c). The V-shape images can be understood by taking into account the dihedral angle between the $\mathrm{C}-\mathrm{F}$ and 
$\mathrm{C}-\mathrm{Br}$ bonds. A stronger covariance signal is present in the bottom of the image because one of the $\mathrm{F}^{+}\left(\mathrm{Br}^{+}\right)$is always ejected in an antiparallel manner relative to the $\mathrm{Br}^{+}\left(\mathrm{F}^{+}\right)$, while the other $\mathrm{F}^{+}\left(\mathrm{Br}^{+}\right)$is ejected in nearly an opposite direction giving rise to weaker signals on either side of the image. These results demonstrate that CEI of aligned molecules is an important technique to obtain information about the molecular structure and, in particular, relative bond angles.

The advantage of utilizing pre-aligned (reduced degree of freedom) molecules in VMI is clearly demonstrated by the studies described above. In order to perform precise structural determination by Coulomb explosion, the degree of alignment must be high. Moreover, reducing the degrees of freedom by orienting molecules (i.e., head-to-tail and tail-to-head discrimination) is helpful for structural determination by CEI. The orientation of molecules in the gas phase has been achieved by taking advantage of interactions between induced dipole moments of molecules and laser electric fields in addition to those between permanent dipole moments of molecules and static electric fields [228-231]. Furthermore, the degree of orientation is strongly enhanced by using the molecules in the lowest-lying rotational states separated by a strong inhomogeneous static electric field before interacting with laser pulses [232-234]. Figure 16 shows that the asymmetry of $\mathrm{I}^{+}$ images for the lowest-lying rotational states (labeled "Deflection") is more significant than that for the ensemble of few rotational states of iodobenzene (labeled "No deflection"). The permanent dipole (iodine end is negative and phenyl end is positive) of iodobenzene is oriented along the electric field ( $E_{\text {stat, }}$, the ion acceleration direction), and thus iodobenzene directs its phenyl end towards the detector (lower electric field side). Then, the orientation of iodobenzene can be controlled by changing the angle $(\beta)$, the polarization direction of orientation laser pulse (EYAG) with respect to the $\mathrm{E}_{\text {stat. }}$. As clearly 
presented in Fig. 16, $\mathrm{I}^{+}$ions are preferentially ejected downwards for $\beta<90^{\circ}$, whereas they are ejected upwards for $\beta>90^{\circ}$.

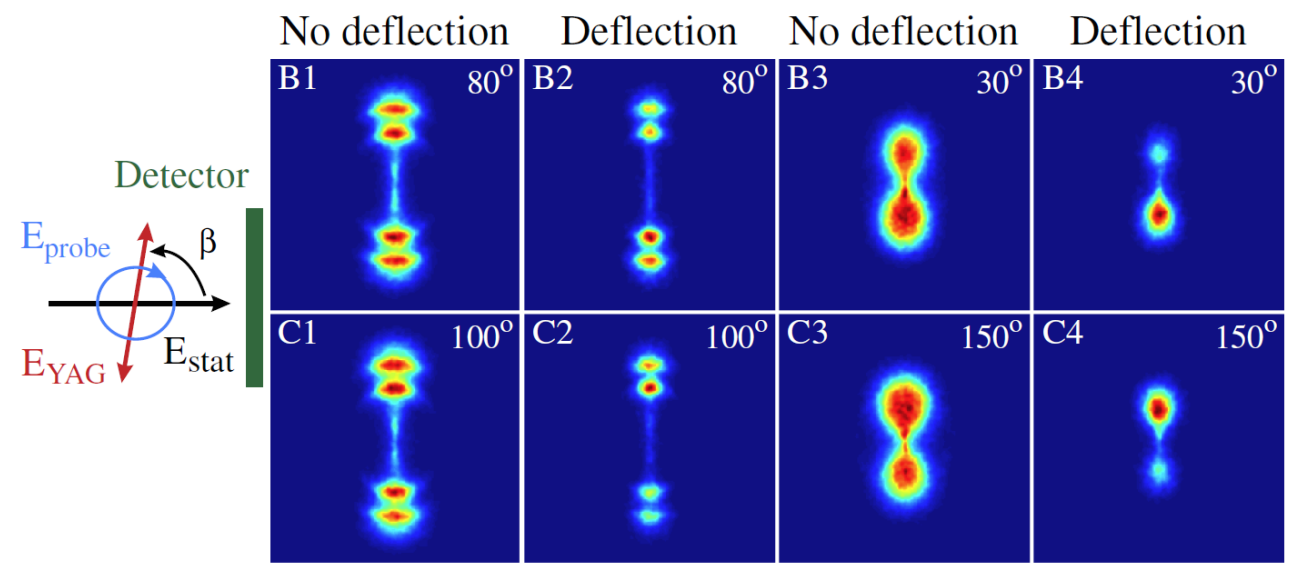

Fig. 16. The images of $\mathrm{I}^{+}$ejected from iodobenzene. Images labeled "No deflection" are obtained for the ensemble of few rotational states. Images labeled "Deflection" are obtained for the lowest-lying rotational states. The polarization direction of orientation laser pulse (EYAG) and the probe pulse $\left(E_{\text {probe }}\right)$ with respect to the detector plane and the static electric field ( $\left.E_{\text {stat }}\right)$ is shown schematically at the left. Figures at the upper right corner of the images are the angle $\beta$. Adopted with permission from Ref.[232]. Copyright 2009 American Physical Society

The combined use of CEI and above mentioned molecular orientation techniques should aid investigations of more complex molecules [234]. In addition, one-dimensional orientation in the absence of static electric fields can be attained by using the interaction between anisotropic hyperpolarizability of molecules and an intense nonresonant twocolor laser field [235]. However, molecules of general interest consist of lighter atoms and hydrocarbon moieties and, thus, improvements in mass resolution may be required in order to determine small mass differences. In addition, the orientation of the molecules having small permanent and induced dipoles as well as small hyperpolarizability is 
challenging. Furthermore, the uncertainty of angular distribution originating in nonaxial recoil of ions should be corrected even if the perfect alignment and/or orientation is achieved. Because the structural reconstruction by CEI is based on the assumption that the fragment ions recoil along the bond of which ion forms, the deviation from axial recoil prevent us from retrieving actual molecular structure. Recently, a method to remove the uncertainty originating in nonaxial recoil of ions is presented [236].

\subsubsection{Geometric isomers}

Application of coincidence VMI of Coulomb explosion to discriminate between geometric isomers (cis and trans) in a mixture of isomeric 1,2-dibromoethenes $\left(\mathrm{C}_{2} \mathrm{H}_{2} \mathrm{Br}_{2}\right)$ was described by Ablikim et al. [237]. MMCs were formed by inner-shell electron ( $\mathrm{Br}$ 3d) excitation using a XUV photon $(140 \mathrm{eV})$ of a synchrotron radiation followed by Auger decay. The molecular structure was reconstructed from the three-fold coincidence of ions $\left(\mathrm{C}_{2} \mathrm{H}_{2}{ }^{+}+{ }^{81} \mathrm{Br}^{+}+{ }^{81} \mathrm{Br}^{+}\right)$. In Figure 17 are shown the counts of three-fold coincidence events as a function of the angle between the momentum vectors of two $\mathrm{Br}^{+}$. The two peaks were assigned as $\mathrm{Br}^{+}$emitted from trans $(\cos \theta=-1,180$ degree $)$ and $\operatorname{cis}(\cos \theta=$ $-0.58,125$ degree) isomers. The ratio of abundances deduced by fitting with two Gaussian functions (shaded areas in Fig. 17) was estimated to be $2.04 \pm 0.07$, which is in agreement with the isomeric composition determined by using gas chromatography (2.06). Thus, the results of this effort proved that the identification and the quantitative analysis of mixed geometric isomers is possible by using the coincidence CEI. In Fig. 18(a) is shown the Newton plot that the momenta of $\mathrm{C}_{2} \mathrm{H}_{2}{ }^{+}$(upper half) and $\mathrm{Br}^{+}$(lower half) in the frame of the momentum of the other $\mathrm{Br}^{+}$indicated by an arrow. The schematics of the momentum vectors of the three fragments are shown in Fig. 18(b) for reference purposes. 
Inspection of the plot demonstrates that two $\mathrm{Br}^{+}$are ejected in the opposite (ca. 157 degree) direction from trans isomer, while $\mathrm{C}_{2} \mathrm{H}_{2}{ }^{+}$remains close to the original location. In contrast, $\mathrm{C}_{2} \mathrm{H}_{2}{ }^{+}$and $\mathrm{Br}^{+}$with similar momenta are emitted from the cis isomer at an angle of ca. 125 degree with respect to each other.

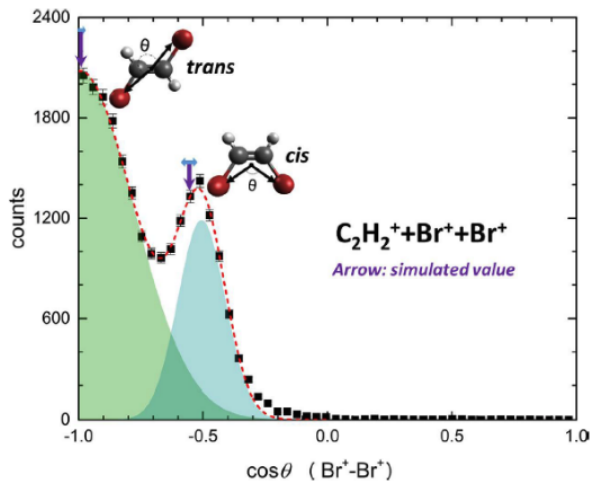

Fig. 17. Three-fold coincidence counts $\left(\mathrm{C}_{2} \mathrm{H}_{2}{ }^{+}+\mathrm{Br}^{+}+\mathrm{Br}^{+}\right)$of 1,2-dibromoethenes as a function of the angle between the $\mathrm{Br}^{+}$ion momenta. Black squares and red dashed line are experimental data and fitted date by two Gaussians (shaded areas), respectively. Reprinted with permission from Ref. [237]. Copyright 2016 Nature Publishing Group. Licensed under a Creative Commons Attribution 4.0 International License.

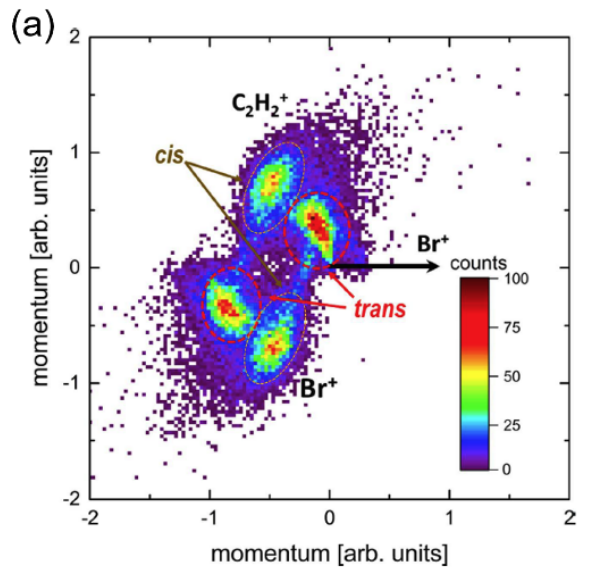

(b)
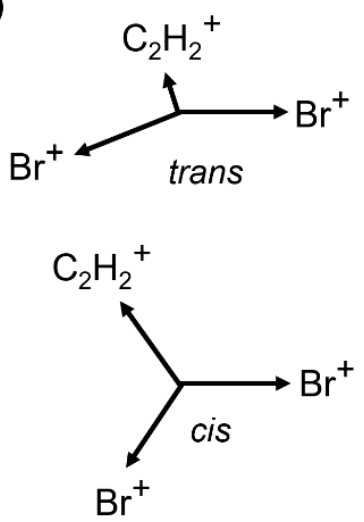

Fig. 18. (a) Newton diagram of the three-fold coincidence channel $\left(\mathrm{C}_{2} \mathrm{H}_{2}^{+}+\mathrm{Br}^{+}+\mathrm{Br}^{+}\right)$ for trans and cis isomers of 1,2-dibromoethenes. Reprinted with permission from Ref. 
[237]. Copyright 2016 Nature Publishing Group. Licensed under a Creative Commons Attribution 4.0 International License. (b) Schematic illustration of momentum vectors of trans (upper) and cis (lower) isomers extracted from the Newton diagram.

Recently the same authors applied a similar strategy to distinguish between the positionally isomeric 3,5- and 2,6-difluoroiodobenzenes $\left(\mathrm{C}_{6} \mathrm{H}_{3} \mathrm{~F}_{2} \mathrm{I}\right)$ [238]. The success of coincidence VMI of Coulomb explosion to distinguish geometric and positional isomers is an important step in investigating isomerization dynamics by using future timeresolved pump-probe experiments.

\subsubsection{Enantiomers}

Recently, several spectroscopic methods including photoelectron circular dichroism [239] and microwave three-wave mixing [240] have been developed to discriminate enantiomers in the gas phase [241]. Kitamura et al. demonstrated that dynamic chirality can be determined by exploring the four-fold coincidence of ions $\left(\mathrm{C}^{2+}\right.$, $\mathrm{D}^{+}, \mathrm{D}^{+}, \mathrm{D}^{+}$) ejected by the Coulomb explosion of $\mathrm{CD}_{4}{ }^{6+}$, which was produced by collision with energetic $\mathrm{Ar}^{8+}[242]$. Pitzer et al. have determined the absolute stereochemistry of $\mathrm{CHBrClF}$ in racemic mixture on a single-molecule basis by using COLTRIMS of a Coulomb explosion induced by femtosecond laser pulses $\left(800 \mathrm{~nm}, 40 \mathrm{fs}, 6 \times 10^{14} \mathrm{~W} \mathrm{~cm}^{-2}\right)$ [243]. Enantiomer discrimination by utilizing CEI has several advantages such as 1) that it can be employed on a single-molecule basis, and it is 2) applicable to gas samples, 3) mass-selective, 4) independent of quantum chemical calculation, and that 5) enantiopure samples are not required.

The chirality of $\mathrm{CHBrClF}$ is judged by the chirality angle $\theta$ defined in eq. (6). 
$\vec{p}_{F} \cdot\left(\vec{p}_{C l} \times \vec{p}_{B r}\right)=\left|\vec{p}_{F}\right|\left|\vec{p}_{C l} \times \vec{p}_{B r}\right| \cos \theta$

, where $\vec{p}_{F}, \vec{p}_{C l}$, and $\vec{p}_{B r}$ are the momenta of $\mathrm{F}^{+}, \mathrm{Cl}^{+}$, and $\mathrm{Br}^{+}$, respectively. The arrows in the inset of Fig. 19 represent $\vec{p}_{F}$ (green), $\vec{p}_{C l}$ (yellow), and $\vec{p}_{B r}$ (red), respectively. The plane defined by $\vec{p}_{C l}$ and $\vec{p}_{B r}$ is indicated by an orange disk. The cross product of $\vec{p}_{C l}$ and $\vec{p}_{B r}$ is represented by a blue arrow, which is normal to an orange disk. The angle between $\vec{p}_{F}$ and $\vec{p}_{C l} \times \vec{p}_{B r}$, hence the inner product of $\vec{p}_{F}$ and $\vec{p}_{C l} \times \vec{p}_{B r}$, gives the chirality angle $\theta$. The $S$ enantiomer of $\mathrm{CHBrClF}$ has obtuse chirality angle $(\cos \theta$ is negative), whereas the $R$ enantiomer has acute chirality angle ( $\cos \theta$ is positive). Consequently, as shown in Fig. 19, the $S$ and $R$ enantiomers can be distinguished from one other. Moreover, the enantiomer excess $(S / R)$ of the mixture of enantiomers can be determined by integrating the counts in the region of $|\cos \theta|>0.6$. The experimental value was determined to be 1.09 , which is in the statistical uncertainty for a racemic sample.

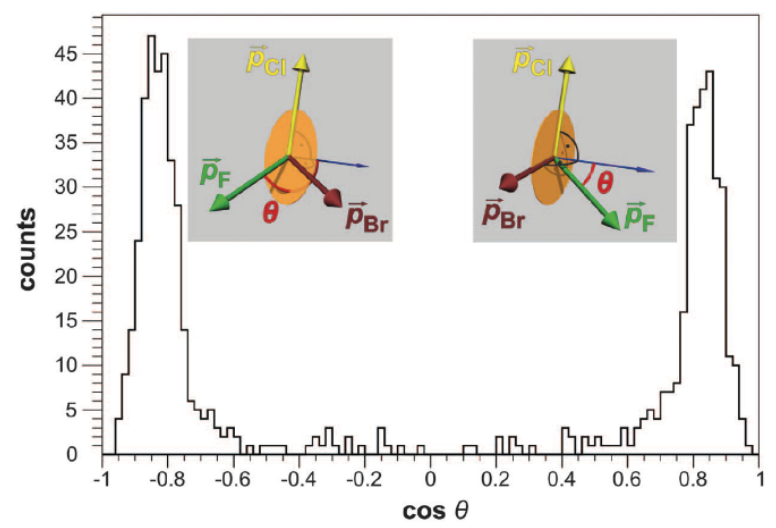

Fig. 19. Five-fold coincidence counts as a function of the chirality angle $\theta$. The peaks at negative and positive $\cos \theta$ correspond to the $S$ and $R$ enantiomers, respectively. Reprinted with permission from Ref. [243]. Copyright 2013 AAAS. 

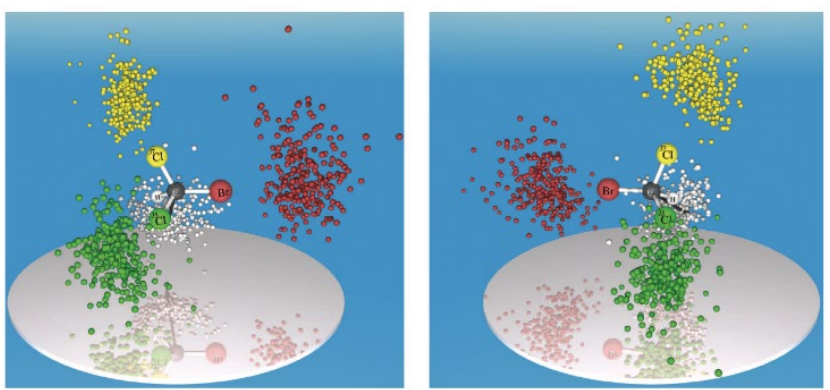

Fig. 20. Linear momenta in five-fold fragmentation of (left) $(S)-\mathrm{CH}^{79} \mathrm{Br}^{37} \mathrm{Cl}^{35} \mathrm{Cl}$ and (right) (R)- $\mathrm{CH}^{79} \mathrm{Br}^{37} \mathrm{Cl}^{35} \mathrm{Cl}$ enantiomers. Reprinted with permission from Ref. [243]. Copyright 2013 AAAS.

Discrimination of the enantiomers of isotopically chiral, $\mathrm{CHBr}^{35} \mathrm{Cl}^{37} \mathrm{Cl}$, was carried out by using five-fold coincidence measurements $\left(\mathrm{C}^{+}+\mathrm{H}^{+}+{ }^{79} \mathrm{Br}^{+}+{ }^{37} \mathrm{Cl}^{+}+{ }^{35} \mathrm{Cl}^{+}\right)$ (Fig. 20). This method gave an enantiomer excess $(S / R)$ of 1.03 , which matches that of an isotopically racemic sample. It is noted that the central carbon, whose momentum is indicated by a gray arrow, is accelerated in the same direction as the proton (momentum is scaled by a factor of 2 and indicated by white spheres), and away from the center of mass due to momentum conservation (i.e., repulsion with slowly moving heavier ions). It should be mentioned that difficulty arises because of the small probability $\left(>10^{-6}\right)$ of fivefold coincidence events. Therefore, long accumulation times are required even when a high repetition rate laser $(100 \mathrm{kHz})$ and an acquisition system $(10 \mathrm{kHz})$ is used. In any event, the use of this method for quantitative discrimination of isotopically chiral molecules on a single-molecule basis demonstrates a great advantage of mass spectrometric detection of VMI.

Recently, CEI of $\mathrm{CHBrClF}$ using a single X-ray photon $(710 \mathrm{eV})$ from a synchrotron light source was reported by the same authors [244]. The results show that 
faster and more accurate determination of absolute configurations are possible as a consequence of the enhancement of coincidence events, faster fragmentations by inner shell excitation followed by Auger decay, and the suppression of linear momentum distribution broadening especially of proton compared with femtosecond laser pulses.

The challenge of the use of CEI to identify chiral molecules is its application in the discrimination of enantiomer of large molecules containing several stereogenic centers. In this case, the probability of chirality-sensitive coincidence events dramatically decreases as the number of fragment pathway increases. Pitzer et al. showed that incomplete fragmentation (partial break-ups), corresponding to four-fold and even threefold coincidence events of five-atom $(\mathrm{CHBrClF})$ [244] and eight-atom $\left(\mathrm{CHClBrCF}_{3}\right)$ molecules [245], can also be used to identify enantiomers. In addition, the same authors examined site-selective excitation of chiral $\mathrm{C}^{*} \mathrm{HClBr}-\mathrm{CF}_{3}$ containing two carbon atoms by varying the photon energy so that only the stereogenic carbon center $\mathrm{C}^{*}(299.0 \mathrm{eV})$ or both carbon atoms $(305.0 \mathrm{eV})$ are excited [245]. The four-body Coulomb explosion involving $\mathrm{CH}^{+}$did not show a photon energy dependence, whereas that involving $\mathrm{H}^{+}$was improved (3.5-9.7 times) when the photon energy was increased.

Pitzer et al. demonstrated that enantiomers arising by the existence of chiral atoms can be distinguished by using CEI. Meanwhile, other types of stereogenic centers including axial, planar, helical, and mechanical chirality can be present in molecules. Axial chirality exists in atropisomeric biaryl compounds, wherein rotation about the arylaryl bond is hindered by large substituents [246]. Christensen et al. have demonstrated that the axial chirality of BP (see above), which is not atropisomeric and thus it has dynamic axial chirality at room temperature, can be determined by using CEI at low temperature [247]. As described in 4.1.1, the discrimination of enantiomers resulting from 
axial chirality is based on the one-dimensional alignment (head-to-tail or tail-to-head) of the chiral axis of molecule along the laser polarization direction of nanosecond laser pulse. Orientational discrimination (head-to-tail and tail-to-head) is made possible by measuring the correlation between a pilot atom, for example, nitrogen of cyano group on the edge of BP, and fluorine and bromine atoms, which are attached to different aryl groups.

The results summarized above show that a major step has been taken in applying Coulomb explosions to determine the absolute configuration of chiral molecules, even two-carbon molecule with a chiral center as well as a large molecule with axial chirality. A great challenge of efforts in this area is the identification of the absolute configurations of more complex chiral molecules as well as biological chiral molecules usually consisting of only lighter atoms such as hydrogen, carbon, nitrogen and oxygen.

\subsection{Molecular complexes and clusters}

Molecular complexes and clusters are aggregates of molecules, which are bounded together by weak van der Waals forces and/or hydrogen bonding interactions. It is well known that carboxylic acids form dimer and phenol forms a trimer in the gas phase. Complexation and clustering of molecules change potential energy surfaces as well as electronic configurations giving rise to photophysical and photochemical properties that are different from those of isolated molecules. These properties are dependent on the size of the clusters or complexes. However, few studies on Coulomb explosion of molecular complexes are found in the literature. Some dimers, such as those of $\mathrm{CO}_{2}$ [248], acetic acid [249], formic acid [250], water [251] and rare gas complexes [252-254] have been investigated using intense femtosecond laser fields.

In contrast, interactions between cluster and intense lasers followed by Coulomb 
explosion have been well studied [255]. For example, photoionization of rare gas clusters has been extensively studied for the purpose of generating X-ray radiation [21]. Recently, ionization of rare gas clusters by utilizing few-cycle femtosecond laser pulses was reviewed by Krishnam et al. [256]. Moreover, ionization of $\mathrm{D}_{2}, \mathrm{H}_{2}$, and $\mathrm{CD}_{4}$ clusters by intense laser pulses has been explored in the context of nuclear fusion and/or neutron generation [257]. Castleman and coworkers have vigorously studied the ionization of metal clusters including those of $\mathrm{Nd}, \mathrm{Eu}$ [258]; U [259]; Ta, Nb, V [260]; Ti, V, Cr, Nb, Ta [261]; Pd, Zr [262]; Nb, Ta [263]; Ni, Pd, Pt [264]; Zr, Pd, W [265]; Ti, V, Cr, Nb, Ta [266]. Transition metals (V, Nb , Ta, Ni, Pd) [267] and their clusters (Ni) [268] have also been studied by Stolow and coworkers. Except for the results of pioneering work described in the late 1990's [208, 269-272] and the early 2000's [206, 209, 273], only few reports exist about the Coulomb explosion of molecular clusters such as aliphatic hydrocarbons [132, 274], haloalkanes [275], and ammonia [276]. Higher kinetic energies and larger charge numbers of atomic ions are produced from these molecular clusters than from isolated molecules. Succeeding ionizations by electrons, which are accelerated by laser electric fields, are dominant energy gain mechanisms in molecular clusters. However, it should be mentioned that Coulomb explosion of clusters followed by the ejection of ions with large charge number and high energy occurs even by using nanosecond laser pulses $[22,23]$. In the cases of the Coulomb explosion processes initiated by using nanosecond and/or picosecond laser pulses, clusters can relax to their most stable structures and, thus, information about the initial configuration is lost. Therefore, we will not discuss details about the ionization and Coulomb explosion of clusters although these processes are interesting in the context of high energy and large charge number fragment ion productions. Instead, we focus on the Coulomb explosion of 
the molecular complexes, which are interesting from the view-point of structure determinations.

\subsection{1. van der Waals complexes}

Molecular complexes, which are weakly bounded by van der Waals force, are one of the interesting and valuable candidates for CEI studies. Since complexes of this type are floppy, the determination of their structures requires special consideration and the results may be highly ambiguous. Microwave rotational spectroscopy is a powerful tool to determine the equilibrium bond lengths and angles in neutral van der Waals complexes. However, structural analysis of multiply charged and floppy van der Waals complexes is beyond the capabilities of any spectroscopic methods. CEI has been successfully applied to the treatment of rare gas complexes such as $\mathrm{N}_{2} \mathrm{Ar}[252,253], \mathrm{O}_{2} \mathrm{Ar}$, $\mathrm{O}_{2} \mathrm{Xe}$ [252] and ArCO [254]. In Figure 21 is shown a Newton diagram of the three-fold coincidence channel of multiply charged $\mathrm{N}_{2} \mathrm{Ar}$ generated by femtosecond laser pulses (780 nm, $25 \mathrm{fs}, 1.3 \times 10^{15} \mathrm{~W} \mathrm{~cm}^{-2}$ ). Charge numbers of $\mathrm{N}$, Ar and $\mathrm{N}$ are indicated in the parenthesis. Three-body Coulomb explosion of this triatomic complex can be clearly identified but a question arises about whether or not the three-body dissociations occur in a direct or sequential manner. Because the complex consists of a weak van der Waals bond between $\mathrm{N}_{2}$ and $\mathrm{Ar}$ and a strong covalent double bond in $\mathrm{N}_{2}$, it is expected that dissociation of the weaker bond occurs before that of stronger bond. Based on the assumption that a sequential dissociation process is involved and it has a time-scale that is comparable to the rotational period of $\mathrm{N}_{2}$, the isotropic (i.e. circle) structure will be reflected in the Newton diagram. However, the observation of a crescent-like structure indicates that the three-body dissociation occurs in a concerted manner, at least within the 
rotational time period.

In contrast, $\mathrm{Wu}$ et al. reported that both direct and sequential dissociation processes occur in the three-body Coulomb explosion of $\mathrm{N}_{2} \mathrm{Ar}^{3+}\left(790 \mathrm{~nm}, 35 \mathrm{fs}, 1.2 \times 10^{15}\right.$ $\mathrm{W} \mathrm{cm}^{-2}$ ) [252]. Circle structures were observed in the Newton diagram, and they are interpreted to be caused by the dissociation of rotating $\mathrm{N}_{2}{ }^{2+}$ into two $\mathrm{N}^{+}$after departure from $\mathrm{Ar}^{+}$. The origin of the differences observed in the above studies is not certain because both experiments were carried out under similar experimental conditions. However, it is possible that intense crescent-like coincidence events may mask the isotropic components, and vice versa.

Neutral $\mathrm{N}_{2} \mathrm{Ar}$ is expected to have a T-shape structure. The second question that arises concerns whether the multiply charged complex retains a structure that is similar to that of the neutral state. It is surprising that $\mathrm{N}_{2} \mathrm{Ar}$ complex retains its T-shape structure up to a charge number corresponding to $\mathrm{N}_{2} \mathrm{Ar}^{6+}$ [253]. In addition, analysis of two-body Coulomb explosion for charge states up to $\mathrm{N}_{2} \mathrm{Ar}^{4+}$ reveal that the distance between the $\mathrm{N}_{2}$ center of mass and Ar at the moment of Coulomb explosion was estimated to be $3.88 \AA$ and independent of the initial charge state and dissociation channels (symmetric or asymmetric charge distributions). 


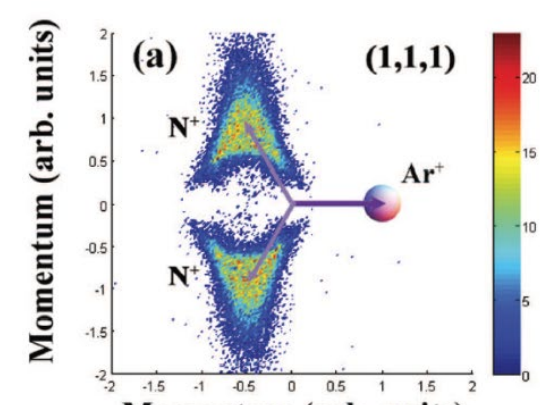

Momentum (arb. units)

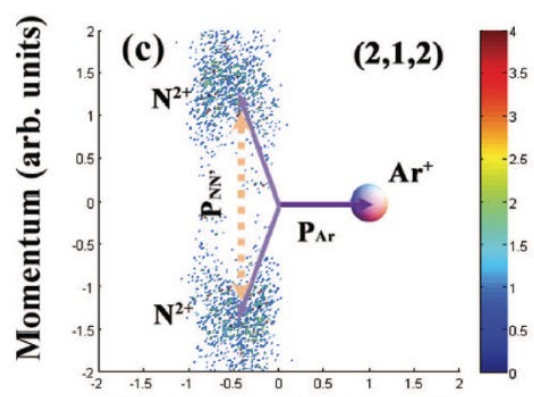

Momentum (arb. units)

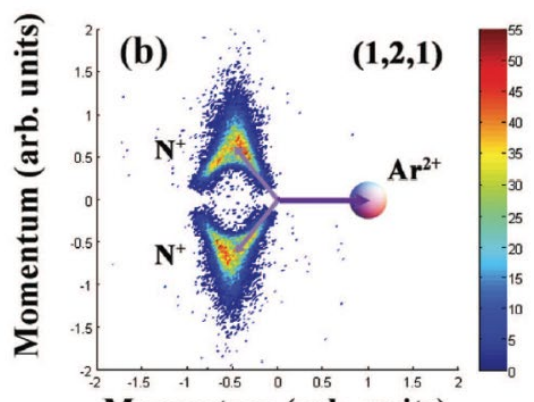

Momentum (arb. units)

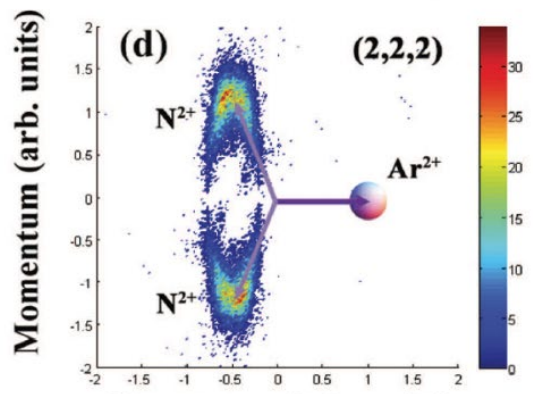

Momentum (arb. units)

Fig. 21. Newton diagram of the three-fold coincidence channels $(a, b, c)$ of $\mathrm{N}_{2} \mathrm{Ar} . \mathrm{a}, \mathrm{b}$, and c denote the charge number of N, Ar, and N, respectively. Adapted with permission from Ref. [253]. Copyright 2014 AIP Publishing LLC.

In addition to the complex of a rare gas atom with a homonuclear diatomic molecule, that of a rare gas with a heteronuclear diatomic molecule is interesting in view of electronic structures, anisotropic intermolecular interactions as well as ionization behaviors. Gong et al. found that the ArCO complex has a tilted T-shape structure, in which argon is closer to the oxygen than to the carbon of $\mathrm{CO}$, and the bond angle between the covalent $\mathrm{CO}$ and van der Waals bonds is 65 degrees [277]. In the CEI of ArCO using COLTRIMS, relative phase controlled elliptically polarized two-color pulses $(790 \mathrm{~nm}$, $7.6 \times 10^{14} \mathrm{~W} \mathrm{~cm}^{-2} ; 395 \mathrm{~nm}, 3.4 \times 10^{14} \mathrm{~W} \mathrm{~cm}^{-2}$ ) was used to achieve orientation selected ionization. In Figure 22 are given the results of the three-body $\left(\mathrm{Ar}^{+}+\mathrm{C}^{+}+\mathrm{O}^{+}\right)$Coulomb explosion of ArCO. Inspection of Fig. 22(a) indicates the existence of both direct (region 
D) and sequential (region S) Coulomb explosion channels, which are identified by kinetic energy releases. Ar carries relatively larger kinetic energy in the first step of sequential Coulomb explosion $\left(\mathrm{ArCO}^{3+} \rightarrow \mathrm{Ar}^{+}+\mathrm{CO}^{2+}\right)$ than it does in the direct Coulomb explosion channel $\left(\mathrm{ArCO}^{3+} \rightarrow \mathrm{Ar}^{+}+\mathrm{C}^{+}+\mathrm{O}^{+}\right)$because of momentum conservation. In the case of the sequential Coulomb explosion channel, the momentum of $\mathrm{Ar}^{+}$(indicated by an orange arrow in Fig. 22(b)) and that of $\mathrm{C}^{+}$and $\mathrm{O}^{+}$are independent since the subsequent Coulomb explosion of long-lived metastable $\mathrm{CO}^{2+}$ is accompanied by its rotation. Therefore, the sequential Coulomb explosion is mapped in a Newton diagram as a circle structure (indicated by a red dashed circle in Fig. 22(b)).
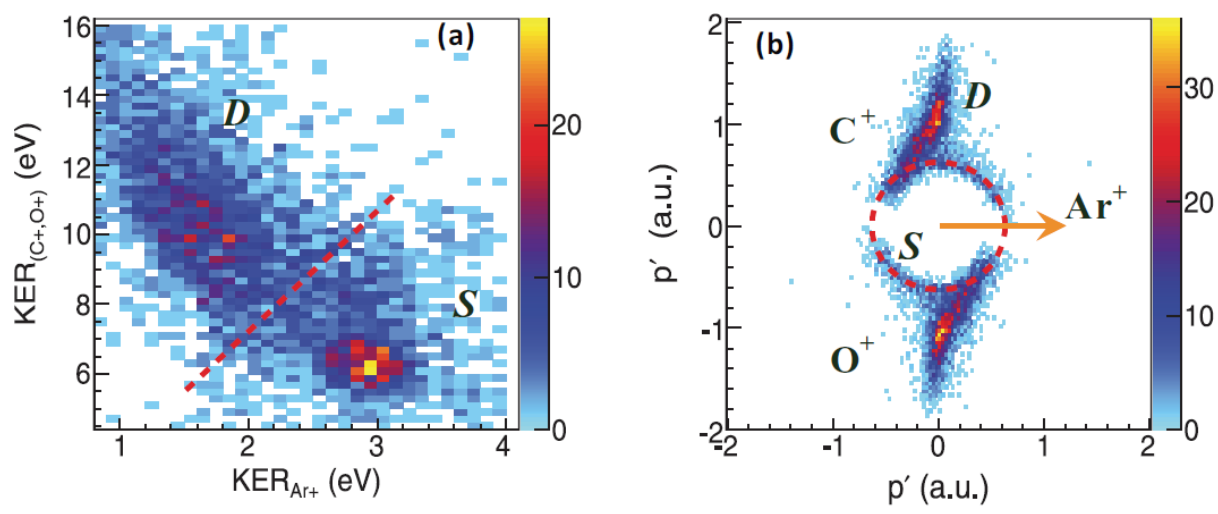

Fig. 22. (a) Distribution of kinetic energy release (KER) of $\mathrm{C}^{+}$and $\mathrm{O}^{+}$as a function of that of $\mathrm{Ar}^{+}$. (b) Newton diagram of the three-fold coincidence channel $\left(\mathrm{Ar}^{+}+\mathrm{C}^{+}+\mathrm{O}^{+}\right)$. Direct and sequential dissociation processes are denoted by $\mathrm{D}$ and $\mathrm{S}$, respectively. Adapted with permission from Ref. [254]. Copyright 2013 American Physical Society.

In contrast to the Newton diagram of $\mathrm{N}_{2} \mathrm{Ar}$, the distribution of $\mathrm{C}^{+}$and $\mathrm{O}^{+}$ momenta in direct Coulomb explosion channel are slightly tilted with $\mathrm{O}^{+}$being closer to $\mathrm{Ar}^{+}$. The initial bond angle $\angle_{(\mathrm{Ar}, \mathrm{CO})}$ and distance $R_{(\mathrm{Ar}, \mathrm{CO})}$ given in Fig. 23 was reconstructed from the asymmetric distribution of momenta by using a molecular 
dynamic simulation. In the simulation, a pure Coulomb potential curve, which is described by the reciprocal of internuclear distance, was not used but instead a repulsive potential curve of ${ }^{3} \Sigma_{g}^{-}$was used for $\mathrm{CO}^{2+}$. Moreover, it was assumed that the direct Coulomb explosion of ArCO complex does not occur in an axial manner as shown in Fig.23. Consequently, an initial bond angle $\angle_{(\mathrm{Ar}, \mathrm{CO})}$ of 65 degree with initial bond distance $R(\mathrm{Ar}, \mathrm{CO})$ of $3.9 \AA$ was obtained.

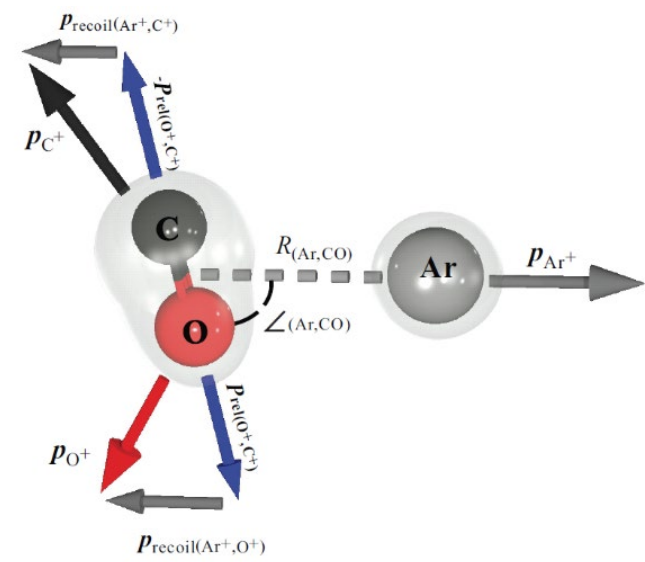

Fig. 23. Schematic illustration of the geometry of ArCO. Reprinted with permission from Ref. [254]. Copyright 2013 American Physical Society.

As demonstrated by using $\mathrm{ArN}_{2}$ and $\mathrm{ArCO}$, the structures of floppy and weakly bounded complexes are analyzed by CEI. Beside the rare gas atom and diatomic molecule complexes mentioned above, Coulomb explosion of other van der Waals complexes have been only seldom explored. Recently, Fan et al. investigated the three-body Coulomb explosion of triply charged $\mathrm{CO}_{2}$ dimer by coincidence VMI $\left(780 \mathrm{~nm}, 25 \mathrm{fs}, 4 \times 10^{14} \mathrm{~W}\right.$ $\left.\mathrm{cm}^{-2}\right)$ [248]. The three-body Coulomb explosion of this species $\left(\mathrm{CO}_{2}^{+}, \mathrm{CO}^{+}, \mathrm{O}^{+}\right)$prefers at least 100 -fold to take place by sequential rather than concerted dissociation. The weak van der Waals bond dissociates to first form $\mathrm{CO}_{2}{ }^{2+}$ and $\mathrm{CO}_{2}{ }^{+}$followed by cleavage of one strong covalent bond in $\mathrm{CO}_{2}{ }^{2+}$ resulting in formation of $\mathrm{CO}^{+}$and $\mathrm{O}^{+}$. 


\subsubsection{Hydrogen-bonded dimers}

As in the cases of the small rare gas van der Waals complexes, hydrogen bonded dimers which are interesting from a physical, chemical, biological and medical viewpoint, have also been only rarely examined. Unlike rare gas complexes, hydrogen bonded dimers of interests are too large to be investigated by using the powerful coincidence VMI technique. Hoshina and coworkers investigated the Coulomb explosion of the hydrogen bonded dimers of formic acid $\left(\mathrm{HCO}_{2} \mathrm{H}\right)$ [250] and acetic acid $\left(\mathrm{CH}_{3} \mathrm{CO}_{2} \mathrm{H}\right)$ [249] dications by using TOF-MS $\left(800 \mathrm{~nm}, 100 \mathrm{fs}, 1 \times 10^{14} \mathrm{~W} \mathrm{~cm}^{-2}\right)$. Dissociation of dimer dications into the corresponding intact monomer cations was demonstrated using momentum matching of ions as well as quantum chemical calculations. Coulomb dissociation into intact molecular cations is a worthwhile process to investigate because Coulomb explosion into atomic ions have been the main focus of studies carried out to date. The mass spectrum of the formic acid dimer is dominated by $\mathrm{H}_{3} \mathrm{O}^{+}, \mathrm{HCO}^{+}, \mathrm{HCOO}^{+}, \mathrm{HCOOH}^{+},(\mathrm{HCOOH}) \mathrm{H}^{+}$, and $(\mathrm{HCOOH}) \mathrm{HCOO}^{+}$at a stagnation pressure of $50 \mathrm{kPa}$ using $\mathrm{He}$ seed gas. The candidate ions that originate from Coulomb explosion were identified by their split peaks as explained in Section 3.1. The ions ejected along the laser polarization direction are $\mathrm{COO}^{+}, \mathrm{HCOO}^{+}\left(\mathrm{DCOO}^{+}\right)$and $\mathrm{HCOOH}^{+}\left(\mathrm{DCOOD}^{+}\right)$. In Figure 24 is compared the momentum distribution of $\mathrm{DCOOD}^{+}$with that of $\mathrm{COO}^{+}$and $\mathrm{DCOO}^{+}$. Neither $\mathrm{COO}^{+}$nor $\mathrm{DCOO}^{+}$are the counterpart of $\mathrm{DCOOD}^{+}$because momentum conservation is not satisfied in the two-body Coulomb explosion of the dimer dication. The kinetic energy release was $3.6 \mathrm{eV}$, which agrees with the value of $3.40 \mathrm{eV}$ estimated by the quantum chemical calculations for the two-body Coulomb explosion of the formic acid dimer dication to produce two intact monomer cations. These findings show that the formic acid dimer is 
doubly ionized prior to hydrogen bond breaking in intense femtosecond laser fields.

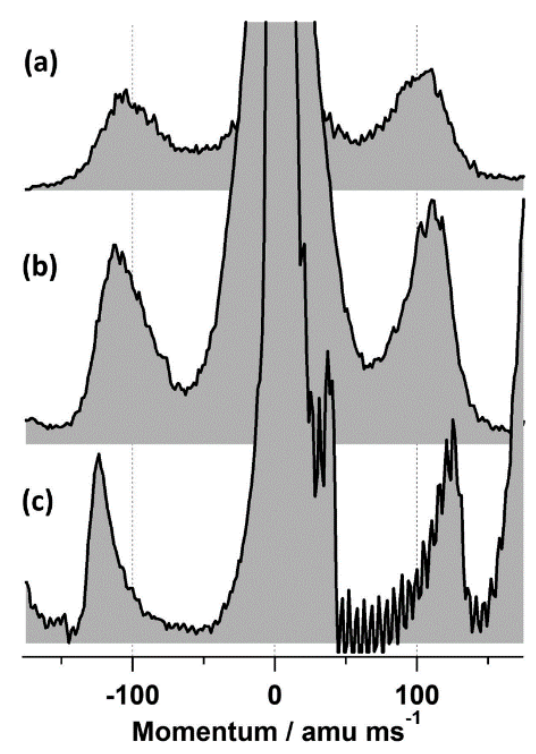

Fig. 24. Momentum-scaled spectra of (a) $\mathrm{COO}^{+}$, (b) $\mathrm{DCCO}^{+}$, and (c) $\mathrm{DCOOD}^{+}$produced from formic acid- $d_{2}$ dimer dication. Reprinted with permission from Ref. [250]. Copyright 2012 American Chemical Society.

The two-body Coulomb explosion of the dimer dication of formic acid is preferred when the laser polarization direction is parallel to the carbon-carbon axis. However, the two-body Coulomb explosion of the acetic acid dimer dication, a homologue of formic acid, occurs when the laser polarization direction is perpendicular to carbon-carbon axis, indicated by $Z_{C} \cdots C$ in Fig. 25 . This observation suggests that deformation of the neutral structure during formation of the metastable and transition states of the dication is preferably induced by applying laser electric field perpendicular rather than parallel to the carbon-carbon axis.

The possible structure and two-body Coulomb explosions of dimer dications of acids resulting in two intact monomer cations can be clearly identified. However, the contribution of this process to the mass spectra is very small compared with other 
dissociation processes. In order to eliminate ambiguities, heterodimers may be promising candidates, and the combination of size-selection [278, 279], stepwise ionization and coincidence measurements could be useful.

The finding outlined above indicate the possibility of applying CEI to the other interesting complexes that have been studied by previously using spectroscopic methods. Potentially interesting targets for this applications are, for example, dimer and trimer of phenol studied by using double resonance spectroscopies [280], and the tripleproton/hydrogen atom transfer in the hydrogen bond network of trimers such as 7azaindole with two methanol [281] or two water molecules [282] studied by fluorescence excitation spectra, REMPI, and theoretical calculations.

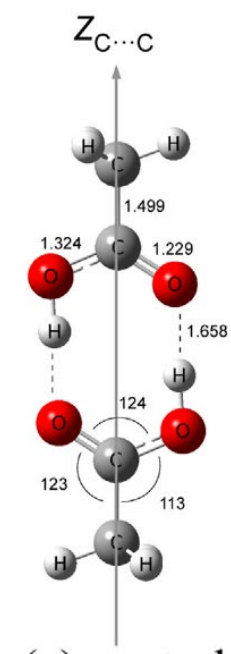

(a) neutral

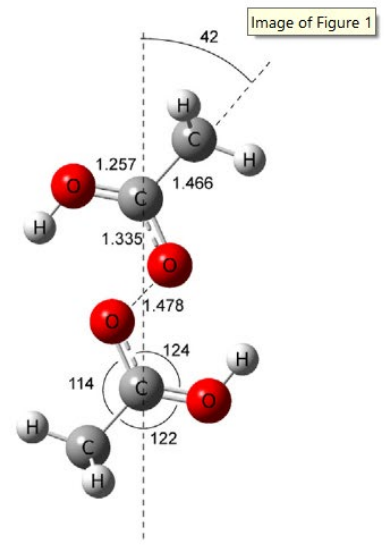

(b) metastable

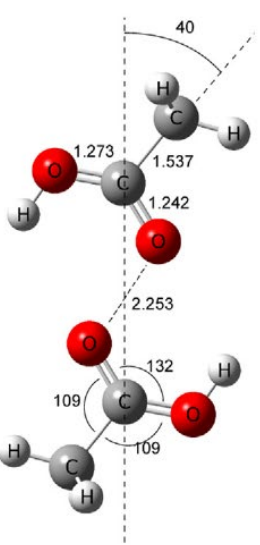

(c) TS

Fig. 25. Calculated stable structure of acetic acid dimer at (a) neutral, (b) metastable dication, and (c) the transition state of dication for dissociation to two monomer cations. Reprinted with permission from Ref. [249]. Copyright 2015 Elsevier.

Experimental studies on the dynamic structural changes of molecular complexes in multiply charged states followed by Coulomb explosion are challenging. Herein, we 
briefly show an example of theoretical calculation. MD simulation of water dimer exposed to intense laser fields was explored by Wang et al [251]. The time evolution of the structures (Fig. 26) and $\mathrm{OH}$ bond lengths (Fig. 27) of the water dimer in intense femtosecond laser fields at different laser intensities $\left(800 \mathrm{~nm}, 8 \mathrm{fs}, 1 \times 10^{14}-3 \times 10^{15} \mathrm{~W}\right.$ $\mathrm{cm}^{-2}$ ) were elucidated by utilizing time-dependent local-density approximation coupled nonadiabatically to MD. Four $\mathrm{OH}$ bonds oscillate with different amplitudes at a laser intensity of $1 \times 10^{14} \mathrm{~W} \mathrm{~cm}^{-2}$, where the number of emitted electron is 0.2 . The oscillation is significant and asynchronous in the hydrogen bond donor $\left(\mathrm{H}_{1}-\mathrm{O}_{1}-\mathrm{H}_{2}\right)$. Further, oscillation of $\mathrm{O}_{1}-\mathrm{H}_{2}\left(3666 \mathrm{~cm}^{-1}\right)$, which forms intermolecular hydrogen bond $\left(\mathrm{H}_{2}-\mathrm{O}_{2}\right)$, precedes that of $\mathrm{O}_{1}-\mathrm{H}_{1}$. The cleavage of the $\mathrm{O}_{1}-\mathrm{H}_{2}$ bond occurs at $4 \times 10^{14} \mathrm{~W} \mathrm{~cm}{ }^{-2}$, where 1.9 electrons are emitted. All $\mathrm{OH}$ bonds show the pattern of Coulomb explosion at $3 \times 10^{15}$ $\mathrm{W} \mathrm{cm}{ }^{-2}$, where 6.5 electrons out of 16 valence electrons are emitted.

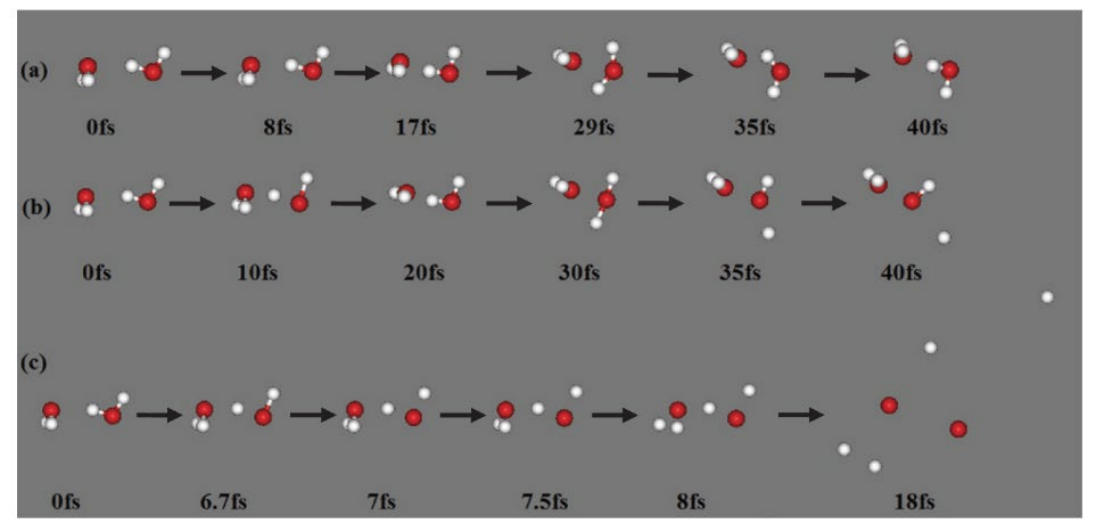

Fig. 26. The time evolution of structure of water dimer exposed to intense laser pulses. (a) $1 \times 10^{14} \mathrm{~W} \mathrm{~cm}^{-2}$, (b) $4 \times 10^{14} \mathrm{~W} \mathrm{~cm}^{-2}$, (c) $3 \times 10^{15} \mathrm{~W} \mathrm{~cm}^{-2}$. Figure reprinted from Ref. [251]. Copyright 2014 Astro Ltd. Reproduced by permission of IOP Publishing Ltd. All rights reserved. 

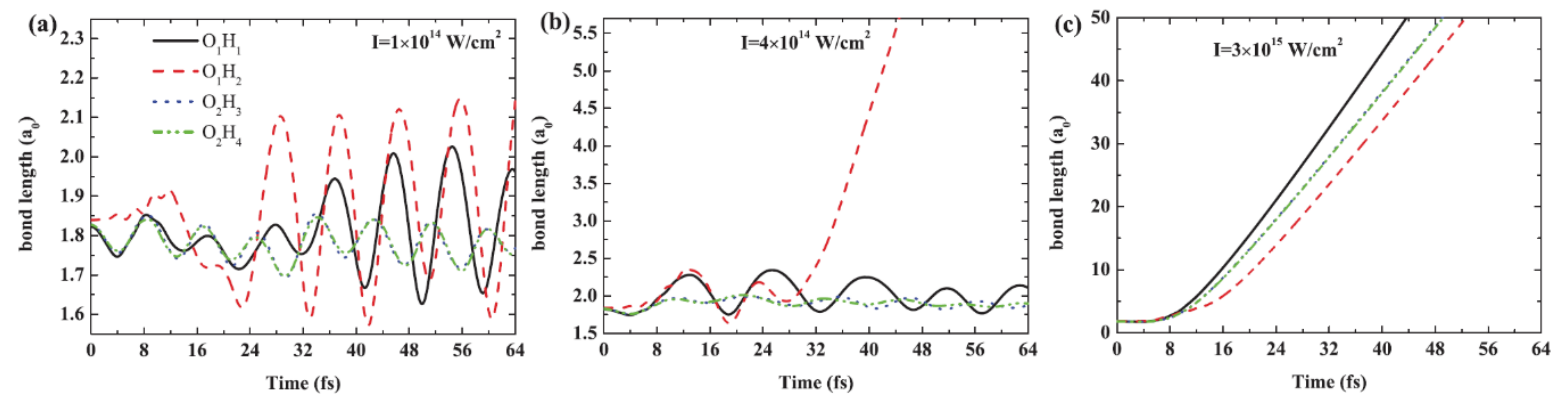

Fig. 27. The time evolution of $\mathrm{OH}$ bond lengths of water dimer exposed to intense laser pulses. (a) $1 \times 10^{14} \mathrm{~W} \mathrm{~cm}^{-2}$, (b) $4 \times 10^{14} \mathrm{~W} \mathrm{~cm}^{-2}$, (c) $3 \times 10^{15} \mathrm{~W} \mathrm{~cm}^{-2}$. Figure reprinted from Ref. [251]. Copyright 2014 Astro Ltd. Reproduced by permission of IOP Publishing Ltd. All rights reserved.

Contrary to the findings coming from experiments with acid dimers, the Coulomb explosion of the water dimer into an intact monomer was not described. The simulation constructed for treating the water dimer revealed that the hydrogen bond donor and its hydrogen bonded $\mathrm{OH}$ bond are more sensitive to the intense laser fields. The simulations produced an important suggestion that the hydrogen bond donor determines the ionization as well as structural changes, and consequently the Coulomb explosion behavior. Thus, it is interesting to investigate hetero-hydrogen bonded complexes either experimentally and theoretically as model systems for biological molecules such as amino acids as well as DNA.

\section{Coulomb explosion of solid surfaces}

Coulomb explosions of isolated molecules, molecular complexes and clusters in the gas phase has been described in the former sections. It is natural to think that in analogy to gas phase experiments multiple ionization of solids should occur at laser 
intensities above $10^{14} \mathrm{~W} \mathrm{~cm}^{-2}$. Generally, the ionization potential of molecules in the solid phase is lower than that in the gas phase. For example, the vertical and adiabatic ionization potentials of fullerene are $7.61 \mathrm{eV}$ in the gas phase, while the work function, threshold (bulk), and peak (near to the surface) ionization potentials of solid fullerene are 4.85, 6.17, and $6.85 \mathrm{eV}$, respectively [283]. The possibility that ablation can be caused by Coulomb explosion was suggested for dielectrics [284, 285], semiconductors [286] as well as metals [287, 288] in 2000's. Debate about this issue has been extensive during past decades [289, 290]. Several processes including Coulomb explosion that lead to material ejection have been proposed and discussed (Fig. 28). The experimental difficulty associated with probing the phenomenon of Coulomb explosion related ablation arises from the fact that the initial and succeeding processes cannot be separated. In addition, the dominance of succeeding thermal ablation processes possibly mask the consequences of the initial Coulomb explosion event. Therefore, it may be difficult to identify the existence of very fast initial Coulomb explosion process by observing the ablation plume and/or resultant solid materials. Experimental observations of morphology changes and discussions about the Coulomb explosion mechanism were described during 2009 and 2016 for silicon [291, 292], metals [293, 294], and dielectrics [295, 296], however, in a strict manner, these events might not give direct evidence for the operation of a Coulomb explosion (electrostatic) process. 


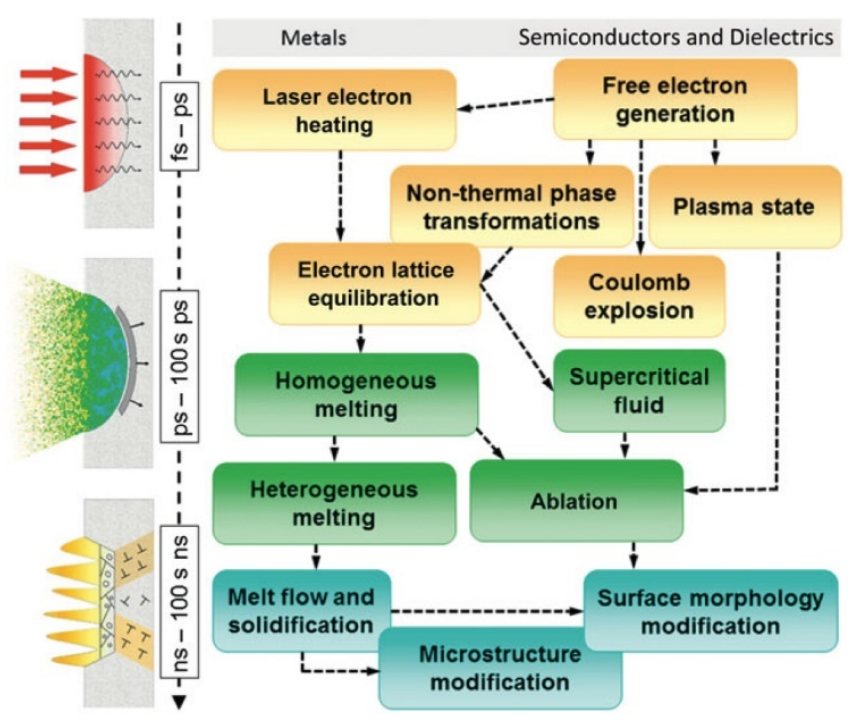

Fig. 28. A schematic of the pathway of energy deposition, dissipation, phase changes and ejection of materials following laser excitation. Reprinted with permission from Ref. [24]. Copyright 2016 Materials Research Society.

It is known that time-resolved reflectivity images give important information about plasma formation [297-299]. TOF measurement, which can identify cationic species, has demonstrated that emission of high energy ions from the solid surfaces takes place. In the early 2000's, it was shown that the existence of electrostatic repulsion in the ablation processes of dielectrics is reflected in the presence of the nonthermal kinetic energy distribution of ejected ions [285, 289], whereas the emission of energetic ions from metals (gold) or semiconductors (silicon) was not observed in those experiments. On the contrary, Sakabe and coworkers proposed that Coulomb explosion is responsible for the self-formation of periodic grating structures on a metal surface [300]. The emission of energetic ions from copper surface was confirmed by using TOF measurements in $2010\left(800 \mathrm{~nm}, 130 \mathrm{fs}, 0.028-2 \mathrm{~J} \mathrm{~cm}^{-2}\right)$ [301]. Furthermore, it was found that the kinetic energy of ions emitted from copper does not follow a Maxwell- 
Boltzmann distribution ( $\left.800 \mathrm{~nm}, 170 \mathrm{fs}, 80-100 \mathrm{~mJ} \mathrm{~cm}^{-2}\right)$ in 2012 [302]. It is not doubted that information about the velocity (momentum) and kinetic energy of emitted ions gives important information about electrostatic interactions, however, this information is the results of the contribution of events that occur over the acceleration time of TOF-MS. Furthermore, care should be taken in experimental results arising from studies of the multiple-laser shots problem. For example, a strong influence of surface morphology on the energy of emitted ions was reported [302]. For a fresh target, the mean energy of copper ions was $50 \mathrm{eV}$ by averaging 100 measurements $(0.08 \mathrm{~J}$ $\mathrm{cm}^{-2}$ ). In contrast, the mean energy of copper ions increased to $180 \mathrm{eV}$ even at the same laser fluence when a preirradiated (600 laser pulses at $\left.0.14 \mathrm{~J} \mathrm{~cm}^{-2}\right)$ target was used. After the prolonged irradiation, the energies of copper ions are reduced and becomes close to that obtained for fresh sample. Experimentally, this enhancement has been explained by invoking a Coulomb explosion of nanostructure formed on the surface by preirradiation [302]. This is important issue but a few studies have concentrated on Coulomb explosions of nanoparticles on substrates [303, 304] or in liquid [28-30].

In the past decades, the importance of ejected electrons and residual positive charges in the ablation process has been recognized. Time-resolved studies carried out by using electron shadow imaging and electron beam deflection [305], and electron deflectometry $[306,307]$ have proven the existence of electric fields caused by emitted electrons and, in principle, residual positive charges on the surface. In this section, we will introduce below the results of some time-resolved studies of electric field applied for semiconductor (silicon), metal (copper), dielectric (sapphire) and polymer. In addition, theoretical calculations about metals carried out since 2014 are briefly mentioned. We emphasize that our main focuses here is on the ablation of carbon based compounds 
including organic molecular crystals and powders that can be compared with the ionization of isolated and cluster form of corresponding molecules.

\subsection{Time-resolved study of the electric fields}

Electric field dynamics were explored using an electron deflectometry by Miller and coworkers in 2008 (silicon, $390 \mathrm{~nm}, 150 \mathrm{fs}, 5.6 \mathrm{~J} \mathrm{~cm}^{-2}$ ) [305] and by Sakabe and coworkers in 2011 (aluminum, $800 \mathrm{~nm}, 200 \mathrm{fs}, 1 \times 10^{16} \mathrm{~W} \mathrm{~cm}^{-2}$ ) [307], using electron shadow imaging and an electron deflectometry by Cao and coworkers in 2011 (copper and sapphire, $800 \mathrm{~nm}, 50 \mathrm{fs}$ ) [306], and using low-energy electron scattering by Greber and coworkers in 2009 (copper, $800 \mathrm{~nm}, 100 \mathrm{fs}, 10^{11} \mathrm{~W} \mathrm{~cm}^{-2}$ ) [308]. It should be noted that a simple comparison of electric field strengths determined in these experiments is difficult because the strengths are measured at different delay times between pump laser pulses and probe electron pulses, different probe positions relative to the pump laser focal spot, and different measurement techniques. In addition, the origins of the electric field are not solely residual positive charges, which are important for Coulomb explosion of cationic species, but also electron clouds. The expanding electron clouds may pull positive species from the surface but such effect is difficult to observe experimentally. Although the electric field generated on the solid surface may not directly be connected to Coulomb explosion of cationic species, the investigations of charges on the solid surface should be worthwhile evidences to present in this review.

Miller and co-workers observed the time evolution of electric fields on a silicon surface with an electron probe beam $(55 \mathrm{keV},>200 \mathrm{fs})$ after a femtosecond pump laser pulse (390 nm, $150 \mathrm{fs}, 5.6 \mathrm{~J} \mathrm{~cm}^{-2}, 17 \mu \mathrm{m}$ in diameter at fwhm) irradiation in 2008 [305]. In Figure 29 is shown a schematic of the electron deflectometry setup. A silicon strip with 
$330 \mu \mathrm{m}$ thickness was exposed to a focused femtosecond pump laser pulse at normal incidence. The probe electron beam impinges the side of the sample, and the deflection of the part of this beam that passes through sample surface, is observed. Integrated images of the probe electron beam, shown in Fig. 30, were interpreted in the following manner. Initially, the probe electron beam is strongly deflected (toward the right) away from the surface due to the space charge of the emitted electron cloud. The probe electron beam then splits into two lobes (3-9.5 ps) caused by the expanding electron cloud, and the electron beams above and below the excitation spot are deflected behind the sample because of the attractive field of the residual positive charges. After expansion of the electron cloud, the probe electron beam returns to the center of the laser focal spot as a single beam. However, the probe electron beam is still deflected toward the sample surface (toward left) after $300 \mathrm{ps}$ as a result of attraction by residual positive charges. The dynamics of probe electron beam images up to 10 ps was well reproduced by numerical simulation. The electric field strength $3 \mathrm{ps}$ after the pump laser pulse was estimated to be $3.5 \times 10^{6} \mathrm{~V} \mathrm{~m}^{-1}$ (at $5.6 \mathrm{~J} \mathrm{~cm}^{-2}$ ) from the maximum deflection of electron probe beam.

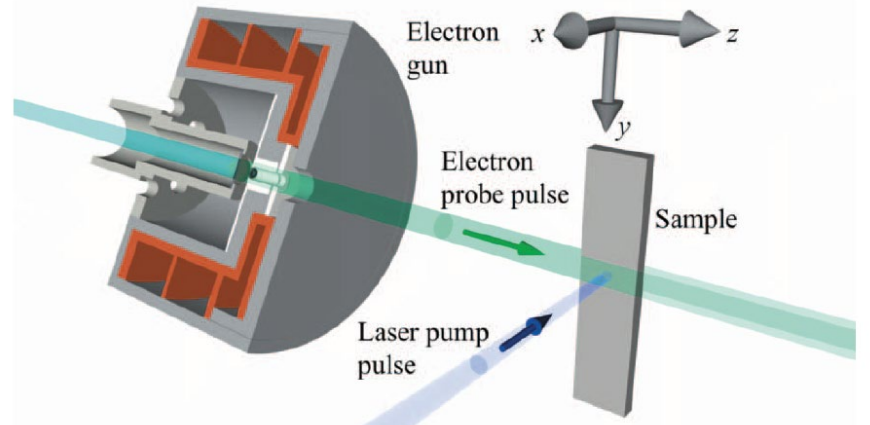

Fig. 29. Schematic of laser pump and electron beam probe experiments on the silicon surface. Reprinted with permission from Ref. [305]. Copyright 2008 American Physical Society. 

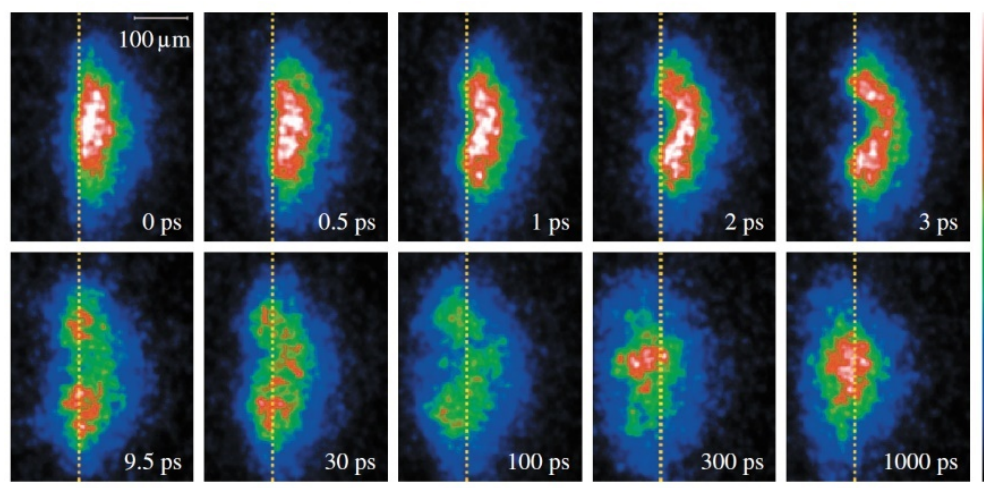

Fig. 30. Time-resolved images of probe electron beam. The surface of silicon strip indicated by yellow line was exposed to laser beam coming from right hand side. Reprinted with permission from Ref. [305]. Copyright 2008 American Physical Society.

In Figure 31(a) are given time evolution of electron shadow images taken by the probe electron beam (40 keV, $300 \mathrm{fs}, 1.5 \mathrm{~mm}$ in diameter) after pump laser excitation (800 nm, $50 \mathrm{fs}, 2.2 \mathrm{~J} \mathrm{~cm}^{-2}, 100 \mu \mathrm{m}$ in diameter) of a $100 \mu \mathrm{m}$ thick copper slab. A semispherical shadow clearly grows at $5 \mathrm{ps}$ and then disappears. Displacement of the reduced-size probe electron beam $(75 \mu \mathrm{m}$ in diameter) pass the points indicated by A and B as a function of the time delay between pump and probe pulses is shown in Fig. 31(b). Positions $\mathrm{A}$ and $\mathrm{B}$ are along with the line of the pump laser beam path, and $120 \mu \mathrm{m}$ away from the front and back surface of the sample, respectively.

The electron beam at point $\mathrm{A}$ is deflected in the positive direction, away from the sample front surface, during the first ten picosecond. Then the probe electron beam suddenly changes its direction toward the sample surface (negative direction). After reaching a negative maximum at around $30 \mathrm{ps}$, the electron beam slowly moves back to the original position. These features are explained in terms of transient electric fields generated both by ejected electrons and residual positive charges confined in a surface layer. In contrast, the probe electron beam passing position B is not affected. Therefore, 
the electric field does not exist on the back surface of the copper slab. In the case of sapphire, the trend of probe electron beam deflection on the front surface is basically similar to that displayed by copper. However, the probe electron beam does not return to the original position even after $1.6 \mathrm{~ns}$. Consequently, it is safe to say that screening in sapphire is weaker than that in copper.

(a)
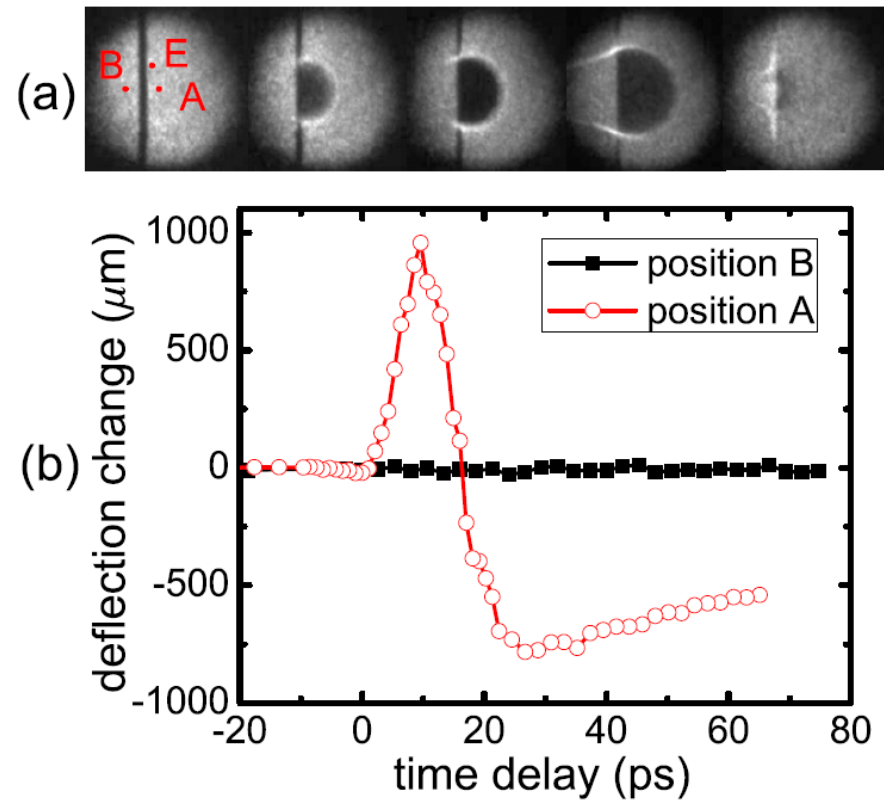

Fig. 31. Time evolution of (a) electron shadow images $0,5,10,26$, and $600 \mathrm{ps}$ after the laser pulse irradiation to copper surface, (b) the center of mass position of the electron beam. Reprinted with permission from Ref. [306]. Copyright 2011 AIP Publishing LLC.

Interestingly, Cao and coworkers compared the electron deflection by copper and sapphire by using two-dimensional deflectometry, in which the electron beam passes the asymmetric line of the laser propagation direction. In Figure 32(a) are shown two-dimensional deflectometry traces for copper and sapphire. Also, for convenience purposes, a schematic illustration of the experimental configuration, laser focal spot (100 $\mu \mathrm{m}$ in diameter), electron beam size ( $75 \mu \mathrm{m}$ in diameter), thickness of sapphire 
$(340 \mu \mathrm{m})$, and the position of electron beam (A, $120 \mu \mathrm{m}$ above the surface and the center of the excitation laser beam; $\mathrm{E}(\mathrm{F}), 40 \mu \mathrm{m}$ above the surface and $140 \mu \mathrm{m}$ from the center of the excitation laser beam), is given in Fig. 32(b). Contrary to one-dimensional deflectometry measurements taken along the line of the pump laser beam path, the electric fields outside of the target area are determined by the anisotropic power balance between the self-expanding electron cloud and residual positive charge confined on the surface. Thus, the two-dimensional traces of the probe electron beam should contain more information about the residual positive charges than the one-dimensional traces.

The time to reach the minimum $Z$ component is 20 ps both in the cases of copper and sapphire. However, the $Z$ component of the probe electron beam remains nearly constant (around $-250 \mu \mathrm{m}$ ) until $480 \mathrm{ps}$ in the case of sapphire, while probe electron beam immediately changes its $Z$ direction in the case of copper at $20 \mathrm{ps}$. In the case of copper, the probe electron beam (E in Fig.32) is deflected nearly perpendicular to the surface, the $\mathrm{Y}$ component of probe electron beam is always positive, and the amplitude of deflection is larger than that of sapphire. These features are expected when a purely repulsive force is operative, i.e., deflection is dominated by ejected electrons and thus the screening in copper is completed. On the contrary, in the case of sapphire, the amplitude of probe electron beam (F in Fig.32) deflection is smaller than in the case of copper, and the direction of the $\mathrm{Y}$ component is changed from positive to negative at $200 \mathrm{ps}$. These features are explained in terms of the existence of a lasting positive charge at the pump laser focal spot that attracts probe electron beam. These observations led to the conclusion that instantaneous shielding (neutralization) of positive charges on copper surface occurs, whereas the residual positive charge on sapphire remains within the excited area for hundreds of picoseconds. Although the interpretation of 
deflectometry is only qualitative, the results offer strong support for the conclusion that Coulomb repulsion between positive charges is the driving force of ionic fragments ejection from the sapphire surface.

(a)

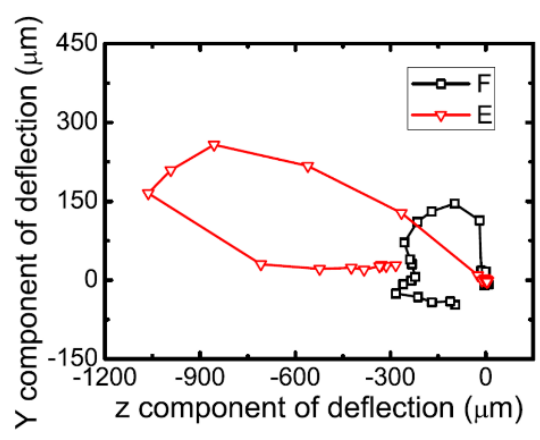

(b)

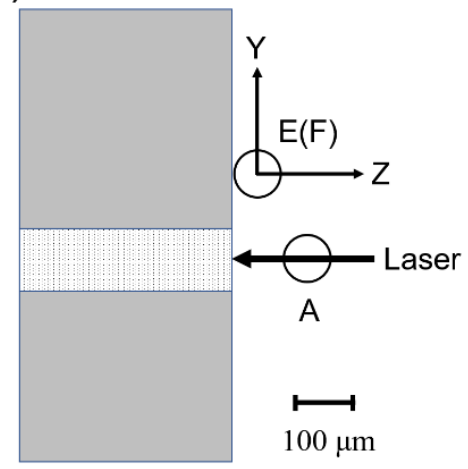

Fig. 32. (a) Two-dimensional deflection traces of copper (E, inverted triangles) and sapphire (F, squares). The traces form counterclockwise loops with increasing time (copper, 0, 4, 8, 12, 16, 20, 60, 100, 140, 180, 300, 700, 1100, and 1500 ps; sapphire, 0, $4,8,12,16,20,28,36,68,108,200,480,880,1280,1680$, and 2080 ps). Reprinted with permission from Ref. [306]. Copyright 2011 AIP Publishing LLC. (b) Schematic illustration of experimental configuration.

Sakabe and coworkers utilized the interaction between two electron clouds to investigate electric field dynamics [307, 309, 310]. A schematic of the time-resolved electron deflectometry setup is displayed in Fig. 33. The pump laser pulse creates both an electron cloud, which propagates isotopically, and positive charges confined on the surface. After a time delay, the probe laser pulse generates another electron cloud close to the spot where the pump laser pulse was focused. The distance between two laser spots on aluminum foil of $12 \mu \mathrm{m}$ thick was varied between $30 \mu \mathrm{m}$ and $240 \mu \mathrm{m}$. Relatively high intensity laser pulses $\left(800 \mathrm{~nm}, 200 \mathrm{fs}, 4 \times 7 \mu \mathrm{m}^{2}, 1 \times 10^{16} \mathrm{~W} \mathrm{~cm}^{-2}\right)$ were used as either the 
pump or probe. The electrons emitted in the target-normal direction were collected, and energy-selected electrons $(120 \mathrm{keV})$ were projected to a fluorescent screen by using an electron lens with high spatial resolution. The image was obtained on a single-shot basis. The deflection of electron cloud, which is created by probe laser pulse, was determined from the displacement of the distance between the two electron cloud images from that between pump laser and probe laser spots. Because the electron clouds are deflected by a Lorentz force, the distance between two electron images becomes shorter or longer when the probe electron cloud is deflected by negative or positive charge. Sakabe and coworkers concluded that the probe electron cloud is deflected by the electron cloud rather than by the residual positive charge because the distance between two electron images is always shorter than the distance between two laser focal spots.

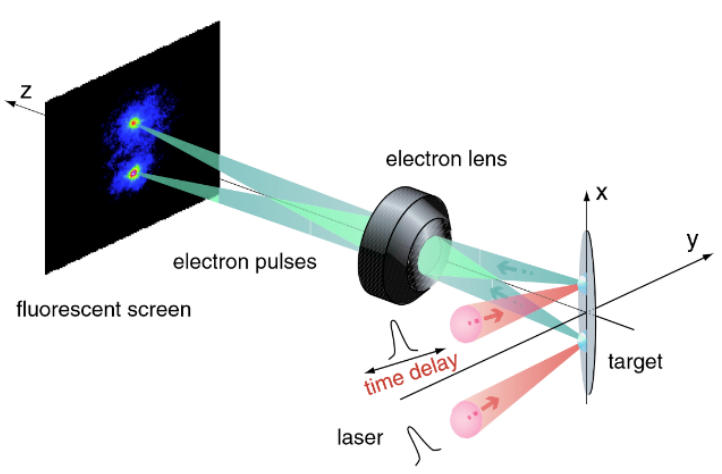

Fig. 33. Schematic of the setup for electron deflectometry. Reprinted with permission from Ref. [310]. Copyright 2012 American Physical Society.

In Figure 34 is shown the deflection of the electron cloud as a function of the delay time between pump and probe laser pulses. As expected, deflection of the two electron clouds is maximized when the time delay is zero. The solid lines in Fig. 34 correspond to curves fitted by using an exponential function to estimate the maximum of electric field and the decay time of the interaction between two electron clouds. In the 
case when the shortest laser spot distance of $30 \mu \mathrm{m}$ is used, deflection of the probe electron cloud was observed to occur between -1 to $1 \mathrm{ps}$, and the decay time was estimated to be $400 \pm 50$ fs. As the distance between the electron clouds increases, the decay time becomes longer. These observations clearly indicate that the electric field that originates from the initially formed electron cloud leaving the sample surface remains several picoseconds and within several hundreds of micrometers. After the certain period, the electric field along the sample surface is diminished. The estimated maximum electric field from Fig. $34(30 \mu \mathrm{m})$ at zero delay time is $2 \times 10^{8} \mathrm{~V} \mathrm{~m}^{-1}$, which is two orders of magnitude larger than that obtained for silicon surface (probed by electron beam, 3 ps after the laser excitation) [305].

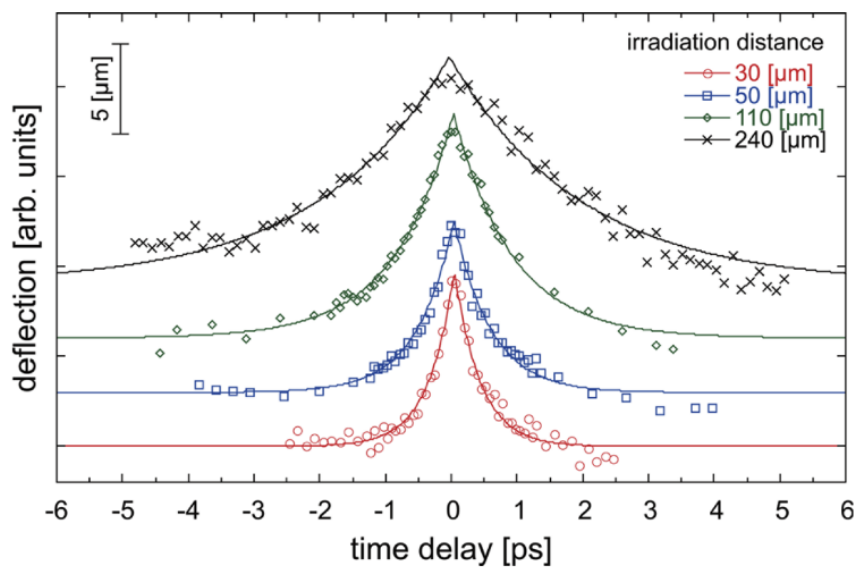

Fig. 34. The deflection of electron cloud as a function of the time delay between pump and probe laser pulses for the different laser spot distances. Reprinted with permission from Ref. [307]. Copyright 2011 AIP Publishing LLC.

In contrast to deflectometry measurements of metal surface, Sakabe and coworkers investigated the deflection of a probe electron cloud by the residual positive charge on polyethylene foil of $10 \mu \mathrm{m}$ thick, which was irradiated by using an ultra-intense 
laser pulse $\left(810 \mathrm{~nm}, 40 \mathrm{fs}, 1.3 \times 10^{18} \mathrm{~W} \mathrm{~cm}^{-2}\right)$ [309]. The striking difference of this pump and probe measurement, compared with that using an aluminum target, is that the ultraintense laser was used to irradiate the front side of the polyethylene foil, and the probe electron cloud was formed on the back side of this foil. Deflection of probe electron beam arising in this manner showed a trend that is opposite to the one using an aluminum target. Deflection of probe electron beam in the polyethylene foil case was explained by the presence of residual positive charge for several hundred picoseconds. The calculated electric field $2.5 \mathrm{~mm}$ away from the laser focal spot was $3.6 \times 10^{6} \mathrm{~V} \mathrm{~m}^{-1}$ [309].

It has been suggested that the Coulomb explosion process is strongly inhibited in metals because the screening (neutralization) effect dominates when a large number of free electrons with large mobility are present. The electron deflectometry experiments carried out on metals support this proposal [306, 307]. However, the results of recent theoretical calculations indicate that the electric fields generated by the femtosecond laser pulse excitation exceed the threshold of Coulomb explosion. Those calculations were presented for aluminum in $2017\left(800 \mathrm{~nm}, 90 \mathrm{fs}, 17.7 \mathrm{~mJ} \mathrm{~cm}^{-2}\right)$ [311] and $2014(800 \mathrm{~nm}$, $\left.90 \mathrm{fs}, 28 \mathrm{~mJ} \mathrm{~cm}^{-2}\right)$ [312], copper in $2015\left(800 \mathrm{~nm}, 100 \mathrm{fs}, 100-400 \mathrm{~mJ} \mathrm{~cm}^{-2}\right)$ [313] and in $2013\left(0.6,0.8,1.0 \mu \mathrm{m}, 70 \mathrm{fs},>10^{19} \mathrm{~W} \mathrm{~cm}^{-2}\right)$ [314], gold in $2014(800 \mathrm{~nm}, 25 \mathrm{fs}, 400$ $\mathrm{mJ} \mathrm{cm}{ }^{-2}$ ) [312], and silver in $2011(800 \mathrm{~nm}, 75 \mathrm{fs})$ [315]. The electron densities, i.e., electric fields, of metals were calculated within the framework of a two-temperature (electron and lattice) heat transfer and electron continuity function, and by using the particle-in-cell method schematically described in Fig. 35. The electric field was calculated by solving the Poisson's equation. 


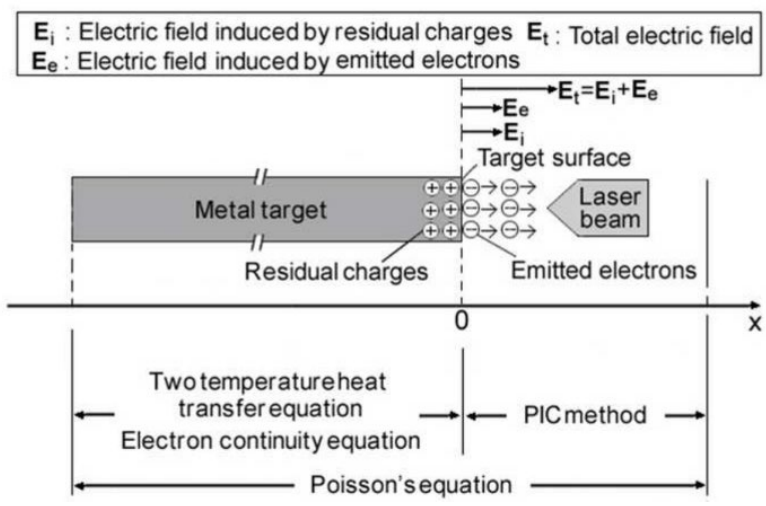

Fig. 35. Schematic of the theoretical model used to calculate electric fields induced by femtosecond laser irradiations. Reprinted with permission from Ref. [312]. Copyright 2014 Elsevier.

Li et al. showed that the electric field strength inside copper reaches the calculated Coulomb explosion threshold $\left(1 \times 10^{10} \mathrm{~V} \mathrm{~m}^{-1}\right)$ at $200 \mathrm{fs}(800 \mathrm{~nm}, 100 \mathrm{fs}, 0.3 \mathrm{~J}$ $\mathrm{cm}^{-2}$, evaluated from Fig. 4 of ref. [313]). The electron density reaches a minimum at about $500 \mathrm{fs}$ and that redistribution of free electron requires about 10 ps [313]. Moreover, the calculations reproduced the experimentally obtained macroscopic ablation depth [316] at laser fluences between 0.35 and $0.8 \mathrm{~J} \mathrm{~cm}^{-2}$. However, a mismatch still exists below $0.3 \mathrm{~J} \mathrm{~cm}^{-2}$, where the calculation gives a zero-ablation depth because the electric field strength is below the Coulomb explosion threshold. This mismatch presumably originates from the relatively high Coulomb explosion threshold $\left(1 \times 10^{10} \mathrm{~V} \mathrm{~m}^{-1}\right)$ derived from cohesive energy, free electron density and permittivity. It should be noted that Hashida et al. have reported the precise ablation rates of copper for laser fluences between $0.02 \mathrm{~J} \mathrm{~cm}^{-2}$ and $20 \mathrm{~J} \mathrm{~cm}^{-2}$ [317]. They have already concluded that Coulomb explosion of copper is operative at $0.1-1.2 \mathrm{~J} \mathrm{~cm}^{-2}(800 \mathrm{~nm}, 130 \mathrm{fs})$ [301].

In summary, we realize that it is not an easy task to discuss the inconsistency of 
the electric field strength obtained experimentally and by using calculations. The calculated Coulomb explosion threshold is $1 \times 10^{10} \mathrm{~V} \mathrm{~m}^{-1}$ for both copper [313] and gold [312]. In contrast, the experimentally reported electric fields are $2 \times 10^{8} \mathrm{~V} \mathrm{~m}^{-1}$ on copper (30 $\mu \mathrm{m}$ away from the laser focal spot probed by electrons at a moment of pump laser excitation, $1 \times 10^{16} \mathrm{~W} \mathrm{~cm}^{-2}$ ) [307], $3.5 \times 10^{6} \mathrm{~V} \mathrm{~m}^{-1}$ on silicon (3 ps after the laser excitation, $\left.5.6 \mathrm{~J} \mathrm{~cm}^{-2}\right)$ [305], and $3.6 \times 10^{6} \mathrm{~V} \mathrm{~m}^{-1}$ on polyethylene foil $(2.5 \mathrm{~mm}$ away from the laser focal spot on the back side) [309]. Because of the physical limitations of probe method such as time and space resolutions, available probe positions relative to the laser focal spot and sensitivity, consistency between theories and experiments is difficult to achieve at this moment.

Further, the electric field strength originated from residual positive charges cannot be investigated in a manner that is independent of emitted electrons because probe electron beam passes through the expanding electron cloud [305-307]. In the case when polyethylene foil is utilized, the existence of residual positive charges is determined by measuring the electric field on the back side of the foil and far away from the laser focal spot on the front side [309]. The long-range goal of studies in this area is to directly correlate the real-time imaging of the expansion of cationic species and the evolution of the electric field generated by the residual positive charges at the pump laser focal spot. The possibility to realize the deflectometry both with positively [318-320] and negatively charged probes is low but the results of experiments of this type could provide complementary results.

\subsection{Carbon based materials}

At the current time, laser ablation is regarded as an indispensable direct sampling 
method in mass spectrometry [321]. The most utilized ionization process for solid materials is matrix-assisted laser desorption ionization (MALDI) [322]. Basically, MALDI is a protonation process instead of electron emission caused by the photoelectric effect. As a result, the emission of ions by MALDI is strongly dependent on the nature of the matrix and its preparation procedure. In addition, the difficulty in exploring the ablation mechanism of solid surface arises from many factors. In the case of analytes deposited on the substrate surface, heating of substrate is not avoidable when laser irradiation is employed. For the cases of metals, semiconductors and dielectrics, bond breaking is required to eject fragments.

The mechanism for ablation of polymers by ultraviolet nanosecond laser pulses has been discussed in detail. Excellent reviews on polymer ablation were presented in 2003 [323-328]. Several ablation mechanisms such as photochemical, photothermal and photomechanical (transient pressure) have been proposed for organic solids and polymers. Detailed time-resolved emission, absorption, interferometric imaging and scattering studies have been employed to detect intermediates and morphological changes [27]. However, the contribution of strong electrostatic fields generated by intense femtosecond pulses has not been considered. As described in 5.1, the Coulomb explosion process has been suggested to be as a strong candidate as a mechanism for ablation of dielectrics and possibly semiconductors. Therefore, it is natural to believe that Coulomb explosion plays a role in the ablation of polymers that are typical insulators.

The Coulomb explosion mechanism has been proposed for carbon-based materials such as graphite and polytetrafluoroethylene (PTFE). Energetic carbon ion emission has been identified as a clue of Coulomb explosion on graphite $(800 \mathrm{~nm}, 100$ fs) [329-331] by Palmer and coworkers, and on PTFE by Hashida et al. (800 $\mathrm{nm}$, 
130-1000 fs, $0.08-40 \mathrm{~J} \mathrm{~cm}^{-2}$ ) [332]. Figure 36 shows a single-shot TOF spectrum of graphite $\left(800 \mathrm{~nm}, 120 \mathrm{fs}, 89 \mathrm{~mJ} \mathrm{~cm}^{-2}\right)$ consisted of a prominent $\mathrm{C}^{+}$and $\mathrm{C}^{2+}$ peak followed by those for medium $\left(\mathrm{C}_{2}{ }^{+}-\mathrm{C}_{27}{ }^{+}\right)$and large $\left(\mathrm{C}_{120}{ }^{+}-\mathrm{C}_{560}{ }^{+}\right)$size carbon clusters [329]. The appearance of multiply charged carbon ions from graphite and PTFE [332] indicates the existence of multiple ionization and electrostatic interactions on the surface.

The ablation of graphite is, however, complicated because this material consists of covalent bonded planes, which are bound each other by van der Waals forces. Therefore, large size carbon clusters as graphene flakes are stripped away from the surface, whereas atomic carbons should be ejected only after covalent $\mathrm{C}-\mathrm{C}$ bonds are broken [330]. Thus, both interlayer and interatom Coulombic interactions need to be considered. The former presumably is simple because ejection could be regarded as two-body or many-body Coulomb explosion of graphene flakes. However, the wide range of size distributions makes this analysis impossible. Thus, the ejection behaviors of carbon ions have been the focus of the studies. The velocities of ejected carbon ions are considerably higher than those expected to be generated thermally. The mean velocities of carbon ions ejected from graphite by femtosecond laser pulse $\left(800 \mathrm{~nm}, 120 \mathrm{fs}, 89 \mathrm{~mJ} \mathrm{~cm}^{-1}\right)$ were $26.52 \times 10^{3}$ and $52.35 \times 10^{3} \mathrm{~m} \mathrm{~s}^{-1}$ for $\mathrm{C}^{+}$and $\mathrm{C}^{2+}$, respectively [329]. These values correspond to kinetic energies of 43.7 and $170 \mathrm{eV}$, respectively. Furthermore, the ratio of the mean velocity (moment) of $\mathrm{C}^{2+}$ to that of $\mathrm{C}^{+}(1.97)$ is consistent with their charge ratio (2). The coincidence of the velocity (momentum) scaling to the charge ratio is strong evidence for the occurrence of Coulomb explosion because the velocity of ions is not necessary dependent on their charges but rather their temperature when thermal ablation process takes place. In the case of multiple-shot experiments, where identical pump and probe pulses $\left(800 \mathrm{~nm}, 100 \mathrm{fs}, 100 \mathrm{~mJ} \mathrm{~cm}{ }^{-2}\right)$ were used, the relative velocities of $\mathrm{C}^{+}, \mathrm{C}^{2+}$, and 
$\mathrm{C}^{3+}$ at zero delay time were $1,2.03$, and 2.97 , respectively [331]. These coincidences support the electrostatic repulsions as a driving force for carbon ion ejection from graphite.

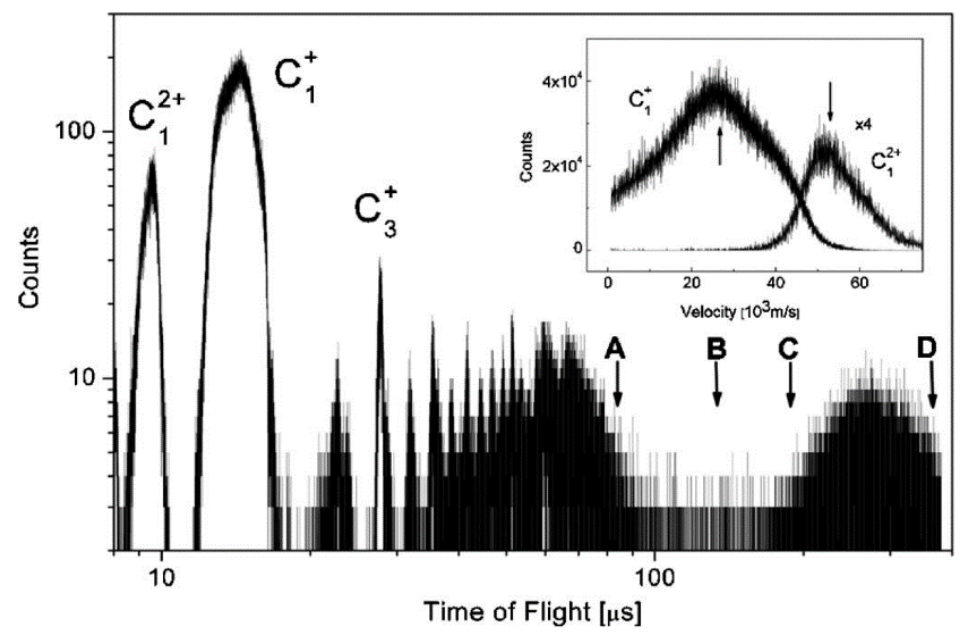

Fig. 36. Single-shot time-of-flight spectra of graphite. Inset shows velocity distribution of $\mathrm{C}^{+}$and $\mathrm{C}^{2+}$. The markers $\mathrm{A}-\mathrm{D}$ indicate the expected flight time of $\mathrm{C}_{27}{ }^{+}, \mathrm{C}_{60}{ }^{+}, \mathrm{C}_{120}{ }^{+}$, and $\mathrm{C}_{580}{ }^{+}$, respectively. Reprinted with permission from Ref. [329]. Copyright 2007 AIP Publishing LLC.

In contrast to metal, semiconductor, dielectrics, polymers and graphite, the binding energy of molecular aggregates is negligibly smaller than Coulomb repulsion energy between cations. Therefore, the comparison of the same molecules in different forms such as isolated, clusters, crystals, and powders will provide straightforward information about the contribution of electrostatic mechanism in laser ablation processes. As a result, we compare the results of studies with isolated $\left(1.4 \mu \mathrm{m}, 130 \mathrm{fs}, 2 \times 10^{13}-\right.$ $\left.2 \times 10^{14} \mathrm{~W} \mathrm{~cm}^{-2}\right)[333]$ and crystalline $\left(1.4 \mu \mathrm{m}, 56 \mathrm{fs}, 5 \times 10^{12}-2 \times 10^{13} \mathrm{~W} \mathrm{~cm}^{-2}\right)$ [334] anthracene exposed to intense femtosecond laser pulses, and anthracene clusters exposed to the energetic projectiles $\left(\mathrm{Xe}^{20+}, 360 \mathrm{keV}\right)[129]$. In the case of isolated anthracene, the triply charged molecular cation is formed with little fragmentation by using a 
femtosecond laser pulses (Fig. 37). Because isolated anthracene is also multiply ionized by collision with energetic $\mathrm{Xe}^{20+}[129]$, it is expected that MMCs should be formed initially in an anthracene cluster. However, the absence of MMCs of anthracene in the case of clusters after the interactions with $\mathrm{Xe}^{20+}$ is clearly seen (Fig. 38). Instead, hydrocarbon fragments, a molecular cation, and $n$-mer cations $(n=1-5$, not shown, please refer Fig.3 in Ref [129]) are formed. In the case of crystalline anthracene, $\mathrm{H}^{+}, \mathrm{C}^{+}$and molecular cation are generated at low laser intensities (Fig. 39 (a)). As the laser intensity increases, MMCs are not seen but rather hydrocarbon fragment ions and dimer and trimer cations are observed (Fig. 39(b)).

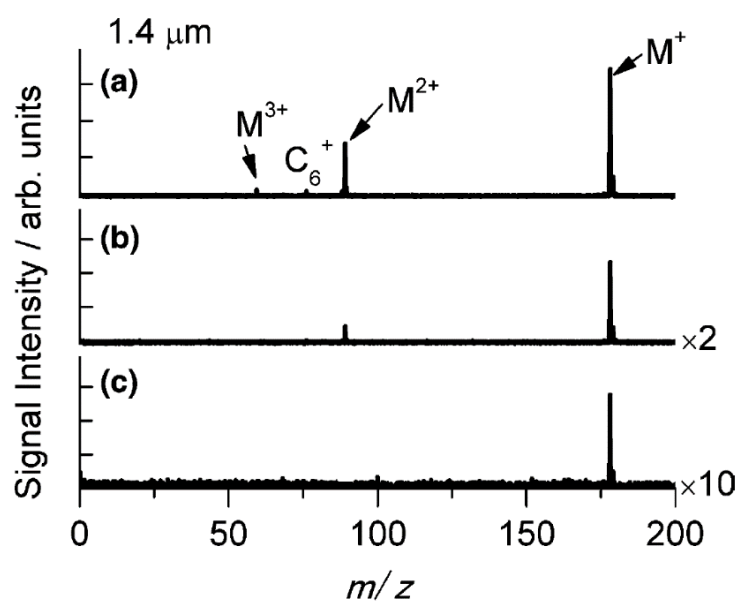

Fig. 37. The mass spectra of isolated anthracene in the gas phase ionized by a $1.4 \mu \mathrm{m}$ pulse at (a) $5.0 \times 10^{13} \mathrm{~W} \mathrm{~cm}^{-2}$, (b) $3.4 \times 10^{13} \mathrm{~W} \mathrm{~cm}^{-2}$, and (c) $1.8 \times 10^{13} \mathrm{~W} \mathrm{~cm}^{-2}$. Symbol $\mathrm{M}^{z^{+}}$indicates the $z$ th charged molecular ion, and $\mathrm{C}_{6}^{+}$indicates $\mathrm{C}_{6} \mathrm{H}_{n}^{+}(n=2,3,4)$. Reprinted with permission from Ref. [333]. Copyright 2005 Elsevier. 


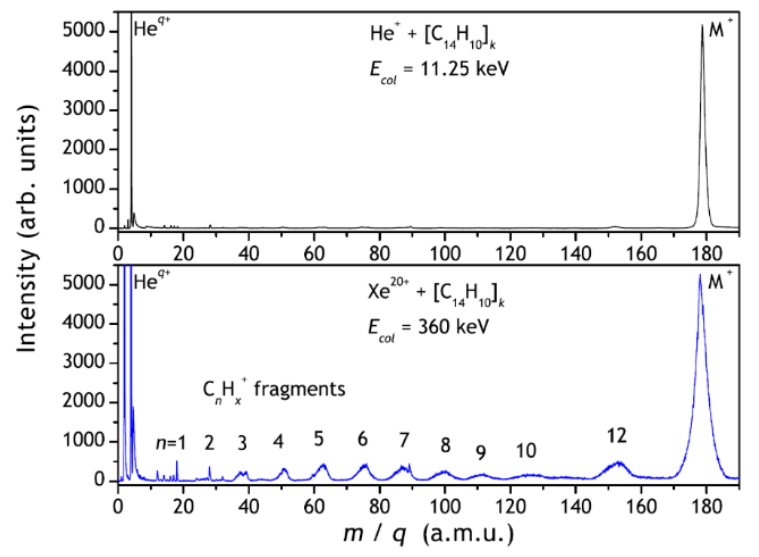

Fig. 38. The mass spectra of anthracene clusters ionized by the collision with energetic $\mathrm{He}^{+}$(upper panel) and $\mathrm{Xe}^{20+}$ (lower panel). Reprinted with permission from Ref. [129]. Copyright 2012 Elsevier.
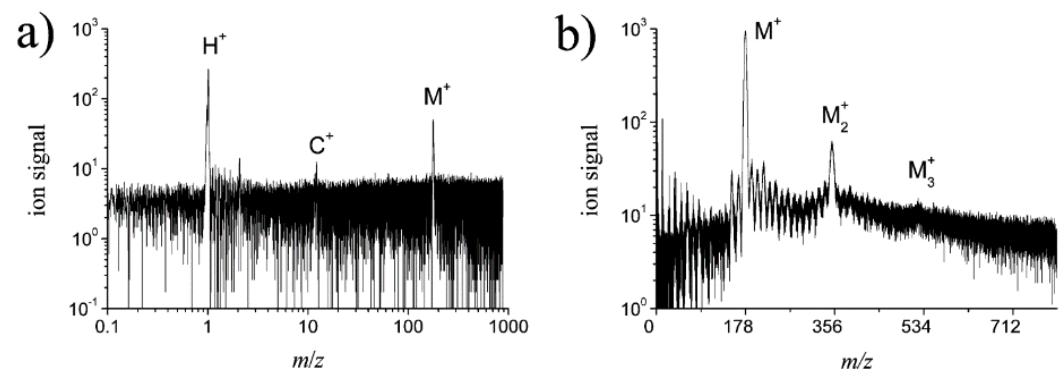

Fig. 39. The mass spectra of crystalline anthracene ionized with $1.4-\mu \mathrm{m}$ pulses at (a) 4.5 $\times 10^{12}$ and (b) $10 \times 10^{12} \mathrm{~W} \mathrm{~cm}^{-2}$. The base signal was not subtracted. $\mathrm{M}^{+}, \mathrm{M}_{2}{ }^{+}$, and $\mathrm{M}_{3}^{+}$ denote the monomer, dimer, and trimer of molecular ions, respectively. Adapted with permission from Ref. [334]. Copyright 2009 American Chemical Society.

The comparison of fullerene ionization behavior by using femtosecond laser pulses and isolated and power forms is also possible. As discussed in section 2.2, isolated fullerene is charged up to +12 , while $\mathrm{C}^{+}$, the molecular, dimer and trimer cations, and fragment ions were observed for solid fullerene $\left(1.4 \mu \mathrm{m}, 58 \mathrm{fs}, 4.7 \times 10^{12}-1.3 \times 10^{13} \mathrm{~W}\right.$ $\mathrm{cm}^{-2}$ ) [335]. Evidently, laser pulse irradiation of isolated molecule results in the formation 
of intact MMCs, while irradiation of its aggregated form results in a singly charged molecular cation and its fragment ions. However, molecules in clusters and crystals should initially be multiply ionized because a sufficient laser intensity as well as projectiles with sufficient energy have been used to promote multiple ionization. Therefore, it is concluded that efficient charge redistribution results in the production of molecular cation radicals in microcrystals, powers and clusters. Because the density of molecular cation radicals is high, they repel each other by Coulomb force in clusters, whereas they react with neutral surroundings in microcrystals and powders. As a consequence, reduction of cluster size and the ejection of aggregated cations from microcrystal and powders is observed.

\section{Summary and perspectives}

Chemical bond dissociations are classified as being homolytic (radical) or heterolytic (ionic) cleavages, in which electrons participating in chemical bond are equally or unequally redistributed in the dissociated pair resulting in neutral radicals or an ion pair. In contrast to those simple reactions, inherently destructive and repulsive nature makes Coulomb explosion of multiply charged species one of the most extreme fragmentation process in chemistry. Nowadays, multiple ionization is a common process that can be promoted by using conventional femtosecond lasers. The facile ability to produce multiply charged species has the potential of enabling the development of new research areas. Owing to their high electron affinity as well as high potential energy, intact multiply charged molecular cations are potential candidates for the next frontier of reactive species. In addition, the high density of electronic states with various spin multiplicities, and charge-dependent reactions, make multiply charged molecular cations 
interesting from both a fundamental and applied scientific perspectives.

The usefulness of multiply charged molecular cations is clearly demonstrated in the success of Coulomb explosion imaging. Coincidence measurements on Coulomb explosions, occurring on multiply charged molecular cations whose repulsion energy well exceeds bond dissociation energies, can be used to determine three-dimensional momenta of fragment ions that can be utilized to reconstruct the molecular structure at the moment of Coulomb explosion. As showcased in this review, significant progress has been made during the past few years in utilizing Coulomb explosion imaging in proof-of-principle demonstrations of molecular structure identifications. Recently, faster and more accurate Coulomb explosion imaging has become possible by using X-ray free electron lasers because in this case the production and dissociation of multiply charged molecular cations is enhanced by inner shell excitation followed by Auger decay. It should be emphasized that the time-resolved Coulomb explosion imaging is an indispensable tool to investigate the ultrafast chemical reaction dynamics of molecules like other developing techniques $[54,336]$.

In contrast to the studies of Coulomb explosion in the gas phase, Coulomb explosion of solids such as metals, dielectrics, semiconductors, polymers and molecular aggregates has been regarded as having minor importance. However, recent time-resolved studies of electric fields have shed light on the origin of the driving force behind ablation processes on solids. It is evident that positive charge exists on the dielectric and polymer surface more than hundreds of picoseconds after femtosecond laser irradiation. Although the majority of ablation has a thermal origin, the occurrence of Coulomb explosion at least in dielectrics and polymers is an issue that should not be ignored. Further investigation about the correlation between the surface charging and cationic species 
ejections will provide fruitful information about the ablation mechanism of solid surface. Furthermore, comparing the ionization behavior of isolated molecules, van der Waals dimers, clusters and aggregates will provide straightforward information about contributions of the electrostatic mechanism to laser ablation of insulators.

\section{Acknowledgements}

This work was supported by JSPS KAKENHI Grant Number JP26107002 in Scientific Research on Innovative Areas "Photosynergetics." 


\section{References}

[1] P. Agostini, L.F. DiMauro, The physics of attosecond light pulses, Rep. Prog. Phys., 67 (2004) 813-855.

[2] M. Nisoli, P. Decleva, F. Calegari, A. Palacios, F. Martin, Attosecond electron dynamics in molecules, Chem. Rev., 117 (2017) 10760-10825.

[3] J. Itatani, J. Levesque, D. Zeidler, H. Niikura, H. Pepin, J.C. Kieffer, P.B. Corkum, D.M. Villeneuve, Tomographic imaging of molecular orbitals, Nature, 432 (2004) 867-871.

[4] H. Niikura, H.J. Worner, D.M. Villeneuve, P.B. Corkum, Probing the spatial structure of a molecular attosecond electron wave packet using shaped recollision trajectories, Phys. Rev. Lett., 107 (2011) 093004.

[5] D.M. Villeneuve, P. Hockett, M.J.J. Vrakking, H. Niikura, Coherent imaging of an attosecond electron wave packet, Science, 356 (2017) 1150-1153.

[6] M. Lein, Molecular imaging using recolliding electrons, J. Phys. B, 40 (2007) R135-R173.

[7] J.L. Xu, C.I. Blaga, P. Agostini, L.F. DiMauro, Time-resolved molecular imaging, J. Phys. B, 49 (2016) 112001.

[8] V.V. Lozovoy, X. Zhu, T.C. Gunaratne, D.A. Harris, J.C. Shane, M. Dantus, Control of molecular fragmentation using shaped femtosecond pulses, J. Phys. Chem. A, 112 (2008) 3789-3812.

[9] X. Xie, E. Lotstedt, S. Roither, M. Schoffler, D. Kartashov, K. Midorikawa, A. Baltuska, K. Yamanouchi, M. Kitzler, Duration of an intense laser pulse can determine the breakage of multiple chemical bonds, Sci.Rep., 5 (2015) 12877.

[10] H.L. Xu, Y. Cheng, S.L. Chin, H.B. Sun, Femtosecond laser ionization and fragmentation of molecules for environmental sensing, Laser Photonics Rev., 9 (2015) 275-293.

[11] J. Kasparian, J.P. Wolf, Physics and applications of atmospheric nonlinear optics and filamentation, Opt. Express, 16 (2008) 466-493.

[12] J. Pisonero, D. Gunther, Femtosecond laser ablation inductively coupled plasma mass spectrometry: fundamentals and capabilities for depth profiling analysis, Mass Spectrom. Rev., 27 (2008) 609-623.

[13] C.P. Wu, A.L. Dill, L.S. Eberlin, R.G. Cooks, D.R. Ifa, Mass spectrometry imaging under ambient conditions, Mass Spectrom. Rev., 32 (2013) 218-243.

[14] M. Malinauskas, A. Zukauskas, S. Hasegawa, Y. Hayasaki, V. Mizeikis, R. Buividas, S. Juodkazis, Ultrafast laser processing of materials: from science to industry, Light. Sci. Appl., 5 (2016) e16133.

[15] R.J. Levis, H.A. Rabitz, Closing the loop on bond selective chemistry using tailored strong field laser pulses, J. Phys. Chem. A, 106 (2002) 6427-6444.

[16] R.J. Levis, G.M. Menkir, H. Rabitz, Selective bond dissociation and rearrangement with optimally tailored, strong-field laser pulses, Science, 292 (2001) 709-713.

[17] A. Assion, T. Baumert, M. Bergt, T. Brixner, B. Kiefer, V. Seyfried, M. Strehle, G. Gerber, Control of chemical reactions by feedback-optimized phase-shaped femtosecond laser pulses, Science, 282 (1998) 919-922.

[18] N. Nakashima, S. Shimizu, T. Yatsuhashi, S. Sakabe, Y. Izawa, Large molecules in high-intensity laser fields, J. Photochem. Photobiol., C, 1 (2000) 131-143.

[19] T.A. Carlson, R.M. White, Measurement of relative abundances and recoil-energy spectra of fragment ions produced as initial consequences of X-ray interaction with $\mathrm{CH}_{3} \mathrm{I}$, HI, and DI, J. Chem. Phys., 44 (1966) 4510-4520. [20] K. Codling, L.J. Frasinski, Coulomb explosion of simple molecules in intense laser fields, Contemp. Phys., 
35 (1994) 243-255.

[21] V.P. Krainov, M.B. Smirnov, Cluster beams in the super-intense femtosecond laser pulse, Phys. Rep., 370 (2002) 237-331.

[22] P. Badani, S. Das, P. Sharma, R.K. Vatsa, Mass spectrometric and charge density studies of organometallic clusters photoionized by gigawatt laser pulses, Mass Spectrom. Rev., 36 (2017) 188-212.

[23] S. Das, P. Sharma, R.K. Vatsa, Tracing photoionisation behaviour of methyl iodide in gas phase: From isolated molecule to molecular aggregate, Journal of Photochemistry and Photobiology C: Photochemistry Reviews, 33 (2017) 27-53.

[24] M.V. Shugaev, C.P. Wu, O. Armbruster, A. Naghilou, N. Brouwer, D.S. Ivanov, T.J.Y. Derrien, N.M. Bulgakova, W. Kautek, B. Rethfeld, L.V. Zhigilei, Fundamentals of ultrafast laser-material interaction, MRS Bull., 41 (2016) 960-968.

[25] B. Rethfeld, D.S. Ivanov, M.E. Garcia, S.I. Anisimov, Modelling ultrafast laser ablation, J. Phys. D, 50 (2017) 193001.

[26] P. Balling, J. Schou, Femtosecond-laser ablation dynamics of dielectrics: basics and applications for thin films, Rep. Prog. Phys., 76 (2013) 036502.

[27] H. Masuhara, Time-resolved spectroscopic and imaging Studies on laser ablation of molecular systems: from mechanistic study to bio/nano applications, Bull. Chem. Soc. Jpn., 86 (2013) 755-783.

[28] L. Delfour, T.E. Itina, Mechanisms of ultrashort laser-induced fragmentation of metal nanoparticles in liquids: numerical insights, J. Phys. Chem. C, 119 (2015) 13893-13900.

[29] D. Werner, A. Furube, T. Okamoto, S. Hashimoto, Femtosecond laser-induced size reduction of aqueous gold nanoparticles: in situ and pump-probe spectroscopy investigations revealing Coulomb explosion, J. Phys. Chem. C, 115 (2011) 8503-8512.

[30] D. Werner, S. Hashimoto, Improved working model for interpreting the excitation wavelength- and fluencedependent response in pulsed laser-induced size reduction of aqueous gold nanoparticles, J. Phys. Chem. C, 115 (2011) 5063-5072.

[31] D. Pentlehner, J.H. Nielsen, A. Slenczka, K. Molmer, H. Stapelfeldt, Impulsive Laser Induced Alignment of Molecules Dissolved in Helium Nanodroplets, Phys. Rev. Lett., 110 (2013) 093002.

[32] B. Shepperson, A.S. Chatterley, A.A. Sondergaard, L. Christiansen, M. Lemeshko, H. Stapelfeldt, Strongly aligned molecules inside helium droplets in the near-adiabatic regime, J. Chem. Phys., 147 (2017) 013946.

[33] L. Christiansen, J.H. Nielsen, L. Christensen, B. Shepperson, D. Pentlehner, H. Stapelfeldt, Laser-induced Coulomb explosion of 1,4-diiodobenzene molecules: Studies of isolated molecules and molecules in helium nanodroplets, Phys. Rev. A, 93 (2016) 023411.

[34] P.E. Mason, F. Uhlig, V. Vanek, T. Buttersack, S. Bauerecker, P. Jungwirth, Coulomb explosion during the early stages of the reaction of alkali metals with water, Nat. Chem., 7 (2015) 250-254.

[35] Z. Vager, R. Naaman, E.P. Kanter, Coulomb explosion imaging of small molecules, Science, 244 (1989) 426431.

[36] P. Herwig, K. Zawatzky, M. Grieser, O. Heber, B. Jordon-Thaden, C. Krantz, O. Novotny, R. Repnow, V. Schurig, D. Schwalm, Z. Vager, A. Wolf, O. Trapp, H. Kreckel, Imaging the absolute configuration of a chiral epoxide in the gas phase, Science, 342 (2013) 1084-1086. 
[37] M. Tarisien, L. Adoui, F. Fremont, D. Lelievre, L. Guillaume, J.Y. Chesnel, H. Zhang, A. Dubois, D. Mathur, S. Kumar, M. Krishnamurthy, A. Cassimi, Ion-induced molecular fragmentation: beyond the Coulomb explosion picture, J. Phys. B, 33 (2000) L11-L20.

[38] S.L. Chin, H.L. Xu, Tunnel ionization, population trapping, filamentation and applications, J. Phys. B, 49 (2016) 1-16.

[39] C. Cornaggia, Ultrafast Coulomb explosion imaging of molecules, Laser Phys., 19 (2009) 1660-1670.

[40] J.H. Posthumus, The dynamics of small molecules in intense laser fields, Rep. Prog. Phys., 67 (2004) 623665.

[41] C. Cornaggia, M. Schmidt, D. Normand, Laser-induced nuclear motions in the Coulomb explosion of $\mathrm{C}_{2} \mathrm{H}_{2}^{+}$ ions, Phys. Rev. A, 51 (1995) 1431-1437.

[42] T. Yatsuhashi, N. Mitsubayashi, M. Itsukashi, M. Kozaki, K. Okada, N. Nakashima, Persistence of iodines and deformation of molecular structure in highly charged diiodoacetylene: anisotropic carbon ion Emission, ChemPhysChem, 12 (2011) 122-126.

[43] C.S. Slater, S. Blake, M. Brouard, A. Lauer, C. Vallance, C.S. Bohun, L. Christensen, J.H. Nielsen, M.P. Johansson, H. Stapelfeldt, Coulomb-explosion imaging using a pixel-imaging mass-spectrometry camera, Phys. Rev. A, 91 (2015) 053424.

[44] J.K. Kou, V. Zhakhovskii, S. Sakabe, K. Nishihara, S. Shimizu, S. Kawato, M. Hashida, K. Shimizu, S. Bulanov, Y. Izawa, Y. Kato, N. Nakashima, Anisotropic Coulomb explosion of $\mathrm{C}_{60}$ irradiated with a high-intensity femtosecond laser pulse, J. Chem. Phys., 112 (2000) 5012-5020.

[45] S. Shimizu, V. Zhakhovskii, F. Sato, S. Okihara, S. Sakabe, K. Nishihara, Y. Izawa, T. Yatsuhashi, N. Nakashima, Coulomb explosion of benzene induced by an intense laser field, J. Chem. Phys., 117 (2002) 31803189.

[46] S. Shimizu, V. Zhakhovskii, M. Murakami, M. Tanaka, T. Yatsuhashi, S. Okihara, K. Nishihara, S. Sakabe, Y. Izawa, N. Nakashima, Coulomb explosion of hexa-fluorobenzene induced by an intense laser field, Chem. Phys. Lett., 404 (2005) 379-383.

[47] K. Lammertsma, P.V. Schleyer, H. Schwarz, Organic dications: gas phase experiments and theory in concert, Angew. Chem. Int. Ed., 28 (1989) 1321-1341.

[48] A. Hishikawa, H. Hasegawa, K. Yamanouchi, Hydrogen migration in acetonitrile in intense laser fields in competition with two-body Coulomb explosion, J. Electron. Spectrosc. Relat. Phenom., 141 (2004) 195-200.

[49] T. Okino, Y. Furukawa, P. Liu, T. Ichikawa, R. Itakura, K. Hoshina, K. Yamanouchi, H. Nakano, Coincidence momentum imaging of ultrafast hydrogen migration in methanol and its isotopomers in intense laser fields, Chem. Phys. Lett., 423 (2006) 220-224.

[50] A. Hishikawa, H. Hasegawa, K. Yamanouchi, Hydrogen migration in acetonitrile in intense laser fields studied by coincidence momentum imaging, Phys. Scr., T110 (2004) 108-111.

[51] R. Itakura, P. Liu, Y. Furukawa, T. Okino, K. Yamanouchi, H. Nakano, Two-body Coulomb explosion and hydrogen migration in methanol induced by intense 7 and 21 fs laser pulses, J. Chem. Phys., 127 (2007) 104306. [52] H. Xu, T. Okino, K. Yamanouchi, Ultrafast hydrogen migration in allene in intense laser fields: evidence of two-body Coulomb explosion, Chem. Phys. Lett., 469 (2009) 255-260.

[53] T. Ando, A. Shimamoto, S. Miura, K. Nakai, H.L. Xu, A. Iwasaki, K. Yamanouchi, Wave packet bifurcation 
in ultrafast hydrogen migration in $\mathrm{CH}_{3} \mathrm{OH}^{+}$by pump-probe coincidence momentum imaging with few-cycle laser pulses, Chem. Phys. Lett., 624 (2015) 78-82.

[54] B. Wolter, M.G. Pullen, A.T. Le, M. Baudisch, K. Doblhoff-Dier, A. Senftleben, M. Hemmer, C.D. Schroter, J. Ullrich, T. Pfeifer, R. Moshammer, S. Grafe, O. Vendrell, C.D. Lin, J. Biegert, Ultrafast electron diffraction imaging of bond breaking in di-ionized acetylene, Science, 354 (2016) 308-312.

[55] T. Yatsuhashi, K. Toyota, N. Mitsubayashi, M. Kozaki, K. Okada, N. Nakashima, Intact four-atom organic tetracation stabilized by charge localization in the gas phase, ChemPhysChem, 17 (2016) 2977-2981.

[56] D. Mathur, Multiply charged molecules, Phys. Rep., 225 (1993) 193-272.

[57] D. Mathur, Structure and dynamics of molecules in high charge states, Phys. Rep., 391 (2004) 1-118.

[58] K. Vekey, Multiply charged ions, Mass Spectrom. Rev., 14 (1995) 195-225.

[59] D.K. Bohme, Multiply-charged ions and interstellar chemistry, Phys. Chem. Chem. Phys., 13 (2011) 1825318263.

[60] D. Schröder, H. Schwarz, Generation, stability, and reactivity of small, multiply charged ions in the gas phase, J. Phys. Chem. A, 103 (1999) 7385-7394.

[61] L. Radom, P.M.W. Gill, M.W. Wong, R.H. Nobes, Multiply-charged cations: remarkable structures and stabilities, Pure Appl. Chem., 60 (1988) 183-188.

[62] D. Schröder, Coulomb explosions and stability of multiply charged ions in the gas phase, Angew. Chem. Int. Ed., 43 (2004) 1329-1331.

[63] R.B. Metz, Optical spectroscopy and photodissociation dynamics of multiply charged ions, Int. J. Mass Spectrom., 235 (2004) 131-143.

[64] S.D. Price, Coincidence studies of the bond-forming reactivity and reaction dynamics of molecular dications, Int. J. Mass Spectrom., 260 (2007) 1-19.

[65] C.M. Gittins, M.J. Castaldi, S.M. Senkan, E.A. Rohlfing, Real-time quantitative analysis of combustiongenerated polycyclic aromatic hydrocarbons by resonance-enhanced multiphoton ionization time-of-flight mass spectrometry, Anal. Chem., 69 (1997) 286-293.

[66] U. Boesl, Multiphoton Excitation and Mass-Selective Ion Detection for Neutral and Ion Spectroscopy, J. Phys. Chem., 95 (1991) 2949-2962.

[67] W. Fuß, W. Rettig, W.E. Schmid, S.A. Trushin, T. Yatsuhashi, Ultrafast temporary charge transfer in pyrrolidinyl-benzonitrile and pyrrolyl-benzonitrile in the gas phase, Faraday Discuss., 127 (2004) 23-33.

[68] W. Fuß, W.E. Schmid, K.K. Pushpa, S.A. Trushin, T. Yatsuhashi, Ultrafast relaxation and coherent oscillations in aminobenzonitriles in the gas phase probed by intense-field ionization, Phys. Chem. Chem. Phys., 9 (2007) 1151-1169.

[69] S.A. Trushin, T. Yatsuhashi, W. Fuß, W.E. Schmid, Coherent oscillations in the charge-transfer system 4dimethylamino-benzonitrile, Chem. Phys. Lett., 376 (2003) 282-291.

[70] T. Yatsuhashi, S.A. Trushin, W. Fuß, W. Rettig, W.E. Schmid, S. Zilberg, Ultrafast charge transfer and coherent oscillations in 4-piperidino-benzonitrile, Chem. Phys., 296 (2004) 1-12.

[71] W. Fuß, W.E. Schmid, S.A. Trushin, Time-resolved dissociative intense-laser field ionization for probing dynamics: femtosecond photochemical ring opening of 1,3-cyclohexadiene, J. Chem. Phys., 112 (2000) 83478362 . 
[72] K. Yamanouchi, Laser chemistry and physics - The next frontier, Science, 295 (2002) 1659-1660.

[73] P. Agostini, F. Fabre, G. Mainfray, G. Petite, N.K. Rahman, Free-free transitions following 6-photon ionization of Xenon atoms, Phys. Rev. Lett., 42 (1979) 1127-1130.

[74] S.L. Chin, F. Yergeau, P. Lavigne, Tunnel ionization of Xe in an ultra-intense $\mathrm{CO}_{2}$ laser field $\left(10^{14} \mathrm{Wcm}^{-2}\right)$ with multiple charge creation, J. Phys. B, 18 (1985) L213-L215.

[75] S. Augst, D.D. Meyerhofer, D. Strickland, S.L. Chin, Laser ionization of noble gases by Coulomb barrier suppression, J. Opt. Soc. Am. B, 8 (1991) 858-867.

[76] B. Walker, B. Sheehy, L.F. Dimauro, P. Agostini, K.J. Schafer, K.C. Kulander, Precision measurement of strong field double ionization of Helium, Phys. Rev. Lett., 73 (1994) 1227-1230.

[77] L.J. Frasinski, K. Codling, P. Hatherly, J. Barr, I.N. Ross, W.T. Toner, Femtosecond dynamics of multielectron dissociative ionization by use of picosecond laser, Phys. Rev. Lett., 58 (1987) 2424-2427.

[78] P.H. Bucksbaum, A. Zavriyev, H.G. Muller, D.W. Schumacher, Softening of the $\mathrm{H}_{2}{ }^{+}$molecular bond in intense laser fields, Phys. Rev. Lett., 64 (1990) 1883-1886..

[79] T. Zuo, A.D. Bandrauk, Charge-resonance-enhanced ionization of diatomic molecular ions by intense lasers, Phys. Rev. A, 52 (1995) R2511-R2514.

[80] T. Seideman, M.Y. Ivanov, P.B. Corkum, Role of Electron Localization in Intense-Field Molecular Ionization, Phys. Rev. Lett., 75 (1995) 2819-2822.

[81] E. Constant, H. Stapelfeldt, P.B. Corkum, Observation of enhanced ionization of molecular ions in intense laser fields, Phys. Rev. Lett., 76 (1996) 4140-4143.

[82] K. Codling, L.J. Frasinski, Dissociative Ionization of Small Molecules in Intense Laser Fields, J. Phys. B, 26 (1993) 783-809.

[83] C. Trump, H. Rottke, M. Wittmann, G. Korn, W. Sandner, M. Lein, V. Engel, Pulse-width and isotope effects in femtosecond-pulse strong-field dissociation of $\mathrm{H}_{2}{ }^{+}$and $\mathrm{D}_{2}{ }^{+}$, Phys. Rev. A, 62 (2000) 063402.

[84] H. Ohmura, T. Nakanaga, Quantum control of molecular orientation by two-color laser fields, J. Chem. Phys., 120 (2004) 5176-5180.

[85] M.J. Dewitt, R.J. Levis, Near-infrared femtosecond photoionization/dissociation of cyclic aromatic hydrocarbons, J. Chem. Phys., 102 (1995) 8670-8673.

[86] K.P. Aicher, U. Wilhelm, J. Grotemeyer, Multiphoton ionization of molecules: a comparison between femtosecond and nanosecond laser pulse ionization efficiency, J. Am. Soc. Mass. Spectrom., 6 (1995) 1059-1068.

[87] K.W.D. Ledingham, H.S. Kilic, C. Kosmidis, R.M. Deas, A. Marshall, T. McCanny, R.P. Singhal, A.J. Langley, W. Shaikh, A comparison of femtosecond and nanosecond multiphoton ionization and dissociation for some nitromolecules, Rapid Commun. Mass Spectrom., 9 (1995) 1522-1527.

[88] K.W.D. Ledingham, R.P. Singhal, High intensity laser mass spectrometry - A review, Int. J. Mass Spectrom. Ion Processes, 163 (1997) 149-168.

[89] M. Lezius, V. Blanchet, M.Y. Ivanov, A. Stolow, Polyatomic molecules in strong laser fields: nonadiabatic multielectron dynamics, J. Chem. Phys., 117 (2002) 1575-1588.

[90] M. Lezius, V. Blanchet, D.M. Rayner, D.M. Villeneuve, A. Stolow, M.Y. Ivanov, Nonadiabatic multielectron dynamics in strong field molecular ionization, Phys. Rev. Lett., 86 (2001) 51-54.

[91] A.N. Markevitch, S.M. Smith, D.A. Romanov, H.B. Schlegel, M.Y. Ivanov, R.J. Levis, Nonadiabatic 
dynamics of polyatomic molecules and ions in strong laser fields, Phys. Rev. A, 68 (2003) 011402.

[92] A.N. Markevitch, D.A. Romanov, S.M. Smith, R.J. Levis, Coulomb explosion of large polyatomic molecules assisted by nonadiabatic charge localization, Phys. Rev. Lett., 92 (2004) 063001.

[93] H. Harada, S. Shimizu, T. Yatsuhashi, S. Sakabe, Y. Izawa, N. Nakashima, A key factor in parent and fragment ion formation on irradiation with an intense femtosecond laser pulse, Chem. Phys. Lett., 342 (2001) 563-570.

[94] H. Harada, M. Tanaka, M. Murakami, S. Shimizu, T. Yatsuhashi, N. Nakashima, S. Sakabe, Y. Izawa, S. Tojo, T. Majima, Ionization and fragmentation of some chlorinated compounds and dibenzo- $p$-dioxin with an intense femtosecond laser pulse at 800 nm, J. Phys. Chem. A, 107 (2003) 6580-6586.

[95] Q.Q. Wang, D. Wu, D.D. Zhang, M.X. Jin, F.C. Liu, H. Liu, Z. Hu, D.J. Ding, H. Mineo, Y.A. Dyakov, Y. Teranishi, S.D. Chao, A.M. Mebel, S.H. Lin, Ionization and dissociation processes of pyrrolidine in intense femtosecond laser field, J. Phys. Chem. C, 113 (2009) 11805-11815.

[96] D. Wu, Q.Q. Wang, X.H. Cheng, M.X. Jin, X.Y. Li, Z. Hu, D. Ding, Effect of cation absorption on ionization/dissociation of cycloketone molecules in a femtosecond laser field, J. Phys. Chem. A, 111 (2007) 94949498.

[97] Q.Q. Wang, D. Wu, M.X. Jin, F.C. Liu, F.F. Hu, X.H. Cheng, H. Liu, Z. Hu, D.J. Ding, H. Mineo, Y.A. Dyakov, A.M. Mebel, S.D. Chao, S.H. Lin, Experimental and theoretical investigations of ionization/dissociation of cyclopentanone molecule in a femtosecond laser field, J. Chem. Phys., 129 (2008) 204302.

[98] M. Tanaka, M. Kawaji, T. Yatsuhashi, N. Nakashima, Ionization and fragmentation of alkylphenols by 0.8$1.5 \mu \mathrm{m}$ femtosecond laser pulses, J. Phys. Chem. A, 113 (2009) 12056-12062.

[99] M. Tanaka, S. Panja, M. Murakami, T. Yatsuhashi, N. Nakashima, Intact molecular ion formation of cyclohexane and 2,3-dimethyl-1,3-butadiene by excitation with a short, intense ferntosecond laser pulse, Chem. Phys. Lett., 427 (2006) 255-258.

[100] M. Murakami, M. Tanaka, T. Yatsuhashi, N. Nakashima, Enhancement of anthracene fragmentation by circularly polarized intense femtosecond laser pulse, J. Chem. Phys., 126 (2007) 104304.

[101] B.J. Pearson, S.R. Nichols, T. Weinacht, Molecular fragmentation driven by ultrafast dynamic ionic resonances, J. Chem. Phys., 127 (2007) 131101.

[102] A.E. Boguslavskiy, J. Mikosch, A. Gijsbertsen, M. Spanner, S. Patchkovskii, N. Gador, M.J.J. Vrakking, A. Stolow, The multielectron ionization dynamics underlying attosecond strong-field spectroscopies, Science, 335 (2012) 1336-1340.

[103] H. Akagi, T. Otobe, A. Staudte, A. Shiner, F. Turner, R. Dorner, D.M. Villeneuve, P.B. Corkum, Laser tunnel ionization from multiple orbitals in HCl, Science, 325 (2009) 1364-1367.

[104] J. Wu, L.P.H. Schmidt, M. Kunitski, M. Meckel, S. Voss, H. Sann, H. Kim, T. Jahnke, A. Czasch, R. Dorner, Multiorbital tunneling ionization of the CO molecule, Phys. Rev. Lett., 108 (2012) 183001.

[105] E. Lötstedt, T. Kato, K. Yamanouchi, Enhanced ionization of acetylene in intense laser fields, Phys. Rev. A, 85 (2012) 041402.

[106] S. Erattupuzha, C.L. Covington, A. Russakoff, E. Lotstedt, S. Larimian, V. Hanus, S. Bubin, M. Koch, S. Grafe, A. Baltuska, X.H. Xie, K. Yamanouchi, K. Varga, M. Kitzler, Enhanced ionisation of polyatomic molecules in intense laser pulses is due to energy upshift and field coupling of multiple orbitals, J. Phys. B, 50 (2017) 125601. [107] M.R. Miller, Y. Xia, A. Becker, A. Jaron-Becker, Laser-driven nonadiabatic electron dynamics in molecules, 
Optica, 3 (2016) 259-269.

[108] S.A. Trushin, W. Fuss, W.E. Schmid, Dissociative ionization at high laser intensities: importance of resonances and relaxation for fragmentation, J. Phys. B, 37 (2004) 3987-4011.

[109] T. Bohinski, K.M. Tibbetts, K. Munkerup, M. Tarazkar, D.A. Romanov, S. Matsika, R.J. Levis, Radical cation spectroscopy of substituted alkyl phenyl ketones via tunnel ionization, Chem. Phys., 442 (2014) 81-85.

[110] T. Bohinski, K.M. Tibbetts, M. Tarazkar, D. Romanov, S. Matsika, R. Levis, Measurement of ionic resonances in alkyl phenyl ketone cations via infrared strong field mass spectrometry, J. Phys. Chem. A, 117 (2013) 12374-12381.

[111] T. Bohinski, K.M. Tibbetts, M. Tarazkar, D. Romanov, S. Matsika, R.J. Levis, Measurement of an electronic resonance in a ground-state, gas-phase acetophenone cation via strong-field mass spectrometry, J. Phys. Chem. Lett., 4 (2013) 1587-1591.

[112] K.M. Tibbetts, T. Bohinski, K. Munkerup, M. Tarazkar, R. Levis, Controlling dissociation of alkyl phenyl ketone radical cations in the strong-field regime through hydroxyl substitution position, J. Phys. Chem. A, 118 (2014) 8170-8176.

[113] T. Bohinski, K.M. Tibbetts, M. Tarazkar, D.A. Romanov, S. Matsika, R.J. Levis, Strong field adiabatic ionization prepares a launch state for coherent control, J. Phys. Chem. Lett., 5 (2014) 4305-4309.

[114] W.L. Zuo, H. Yin, X.C. Liu, H. Lv, L. Zhao, Y. Shi, B. Yan, M.X. Jin, D.J. Ding, H.F. Xu, Identification of the cationic excited state of cyclopentanone via time-resolved Ion yield measurements, Chem. Phys. Lett., 654 (2016) 18-22.

[115] H. Sakai, H. Stapelfeldt, E. Constant, M.Y. Ivanov, D.R. Matusek, J.S. Wright, P.B. Corkum, Metastable triply charged diatomic molecules produced with femtosecond pulses, Phys. Rev. Lett., 81 (1998) 2217-2220.

[116] K. Franzreb, J. Hrusak, M.E. Alikhani, J. Lorincik, R.C. Sobers, P. Williams, Gas-phase diatomic trications of $\mathrm{Se}_{2}{ }^{3+}, \mathrm{Te}_{2}{ }^{3+}$, and $\mathrm{LaF}^{3+}$, J. Chem. Phys., 121 (2004) 12293-12302.

[117] V. Brites, K. Franzreb, J.N. Harvey, S.G. Sayres, M.W. Ross, D.E. Blumling, A.W. Castleman, M. Hochlaf, Oxygen-containing gas-phase diatomic trications and tetracations: $\mathrm{ReO}^{z+}, \mathrm{NbO}^{z+}$ and $\mathrm{HfO}^{z+}(z=3,4)$, Phys. Chem. Chem. Phys., 13 (2011) 15233-15243.

[118] E.W. Muller, S.V. Krishnaswamy, S.B. Mclane, Atom-probe fim analysis of interaction of imaging gas with surface, Surf. Sci., 23 (1970) 112-129.

[119] M.W. Wong, R.H. Nobes, L. Radom, Remarkably stable trications and tetracations - the triheliomethyl trication $\left(\mathrm{CHe}_{3}{ }^{3+}\right)$ and tetraheliomethane tetracation $\left(\mathrm{CHe}_{4}{ }^{4+}\right)$, J. Chem. Soc. Chem. Comm., (1987) 233-234.

[120] H. Hammami, O. Yazidi, M.B. Rhouma, M.M. Al Mogren, M. Hochlaf, Theoretical investigations of the $\mathrm{IO},,^{q+}(q=2,3,4)$ multi-charged ions: Metastability, characterization and spectroscopy, J. Chem. Phys., 141 (2014) 014302 .

[121] M.M. Bursey, P.F. Rogerson, J.M. Bursey, Quadruply charged ions in mass spectrum of ovalene, Org. Mass Spectrom., 4 (1970) 615-617.

[122] R.G. Kingston, M. Guilhaus, A.G. Brenton, J.H. Beynon, Multiple ionization, charge separation and charge stripping reactions involving polycyclic aromatic compounds, Org. Mass Spectrom., 20 (1985) 406-412.

[123] A. Lawicki, A.I.S. Holm, P. Rousseau, M. Capron, R. Maisonny, S. Maclot, F. Seitz, H.A.B. Johansson, S. Rosen, H.T. Schmidt, H. Zettergren, B. Manil, L. Adoui, H. Cederquist, B.A. Huber, Multiple ionization and 
fragmentation of isolated pyrene and coronene molecules in collision with ions, Phys. Rev. A, 83 (2011) 022704. [124] T. Yatsuhashi, N. Nakashima, Formation and fragmentation of quadruply charged molecular ions by intense femtosecond laser pulses, J. Phys. Chem. A, 114 (2010) 7445-7452.

[125] N. Mitsubayashi, T. Yatsuhashi, H. Tanaka, S. Furukawa, M. Kozaki, K. Okada, N. Nakashima, Anisotropic Coulomb explosion of acetylene and diacetylene derivatives, Int. J. Mass Spectrom., 403 (2016) 43-52.

[126] K.W.D. Ledingham, R.P. Singhal, D.J. Smith, T. McCanny, P. Graham, H.S. Kilic, W.X. Peng, S.L. Wang, A.J. Langley, P.F. Taday, C. Kosmidis, Behavior of polyatomic molecules in intense infrared laser beams, J. Phys. Chem. A, 102 (1998) 3002-3005.

[127] L. Robson, A.D. Tasker, K.W.D. Ledingham, P. McKenna, T. McCanny, C. Kosmidis, P. Tzallas, D.A. Jaroszynski, D.R. Jones, Ionisation and fragmentation dynamics of laser desorbed polycyclic aromatic hydrocarbons using femtosecond and nanosecond post-ionisation, Int. J. Mass Spectrom., 220 (2002) 69-85.

[128] T. Yatsuhashi, N. Nakashima, Effects of polarization of $1.4 \mu \mathrm{m}$ femtosecond laser pulses on the formation and fragmentation of naphthalene molecular ions compared at the same effective ionization intensity, J. Phys. Chem. A, 109 (2005) 9414-9418.

[129] P. Rousseau, A. Lawicki, A.I.S. Holm, M. Capron, R. Maisonny, S. Maclot, E. Lattouf, H.A.B. Johansson, F. Seitz, A. Mery, J. Rangama, H. Zettergren, S. Rosen, H.T. Schmidt, J.Y. Chesnel, A. Domaracka, B. Manil, L. Adoui, H. Cederquist, B.A. Huber, Low-energy ions interacting with anthracene molecules and clusters, Nucl. Instrum. Meth. B, 279 (2012) 140-143.

[130] F. Seitz, A.I.S. Holm, H. Zettergren, H.A.B. Johansson, S. Rosen, H.T. Schmidt, A. Lawicki, J. Rangama, P. Rousseau, M. Capron, R. Maisonny, A. Domaracka, L. Adoui, A. Mery, B. Manil, B.A. Huber, H. Cederquist, Polycyclic aromatic hydrocarbon-isomer fragmentation pathways: Case study for pyrene and fluoranthene molecules and clusters, J. Chem. Phys., 135 (2011) 064302.

[131] V.R. Bhardwaj, P.B. Corkum, D.M. Rayner, Internal laser-induced dipole force at work in $\mathrm{C}_{60}$ molecule, Phys. Rev. Lett., 91 (2003) 203004.

[132] A.S. Zaag, O. Yazidi, N.E. Jaidane, M.W. Ross, A.W. Castleman, M.M. Al Mogren, R. Linguerri, M. Hochlaf, Structure, reactivity, and fragmentation of small multi-charged methane clusters, J. Phys. Chem. A, 120 (2016) 1669-1676.

[133] It is likely that a misassignment of ions occurred in the TOF spectra of methane cluster. The assigned peaks of $\mathrm{CH}_{4}{ }^{5+}, \mathrm{CH}_{4}{ }^{4+}, \mathrm{CH}_{4}{ }^{3+}, \mathrm{C}^{2+}$, and $\left(\mathrm{CH}_{4}\right)_{2}{ }^{5+}$ might be those of $\mathrm{C}^{4+}, \mathrm{C}^{3+}, \mathrm{C}^{2+}, \mathrm{CH}^{2+}$, and $\mathrm{CH}_{2}{ }^{2+}$, respectively.

[134] S. De, H. Tezuka, P. Bhatt, G. Vesapidze, C.P. Safvan, J. Matsumoto, H. Shiromaru, Do linear molecules always dissociate along their axis? Intra-molecular scattering within Diiodoacetylene, J. Phys. Conf. Ser., 635 (2015) 032061.

[135] A. Kitashoji, T. Yoshikawa, A. Fujihara, S. Nashima, T. Yatsuhashi, Investigation of multiply charged aromatic cation chemistry by using newly constructed Bradbury-Nielsen ion gate, in: 65th Annual Conference on Mass Spectrometry, Japan, Tsukuba, Japan, 2017, pp. 2 P-56.

[136] A. Kitashoji, T. Yoshikawa, A. Fujihara, T. Kamamori, S. Nashima, T. Yatsuhashi, Selection of a Single Isotope of Multiply Charged Xenon $\left({ }^{A} \mathrm{Xe}^{z^{+}}, A=128-136, z=1-6\right)$ by Using a Bradbury-Nielsen Ion Gate, ChemPhysChem, 18 (2017) 2007-2011.

[137] R.J. Levis, M.J. DeWitt, Photoexcitation, ionization, and dissociation of molecules using intense near- 
infrared radiation of femtosecond duration, J. Phys. Chem. A, 103 (1999) 6493-6507.

[138] I.V. Hertel, T. Laarmann, C.P. Schulz, Ultrafast excitation, ionization, and fragmentation of C 60 , Adv Atom Mol Opt Phy, 50 (2005) 219-286.

[139] J.O. Johansson, E.E.B. Campbell, Probing excited electronic states and ionisation mechanisms of fullerenes, Chem. Soc. Rev., 42 (2013) 5661-5671.

[140] Y. Nabekawa, Y. Furukawa, T. Okino, A.A. Eilanlou, E.J. Takahashi, K. Yamanouchi, K. Midorikawa, Sub10 -fs control of dissociation pathways in the hydrogen molecular ion with a few-pulse attosecond pulse train, Nat. Commun., 7 (2016) 12835.

[141] H. Wu, S.A. Zhang, J. Zhang, Y. Yang, L. Deng, T.Q. Jia, Z.G. Wang, Z.R. Sun, Observation of hydrogen migration in cyclohexane under an intense femtosecond laser field, J. Phys. Chem. A, 119 (2015) 2052-2057.

[142] J. Strohaber, F. Zhu, A.A. Kolomenskii, H.A. Schuessler, Observation of anisotropic fragmentation in methane subjected to femtosecond radiation, Phys. Rev. A, 89 (2014) 023430.

[143] Z.F. Wu, C.Y. Wu, Q.Q. Liang, S.F. Wang, M. Liu, Y.K. Deng, Q.H. Gong, Fragmentation dynamics of methane by few-cycle femtosecond laser pulses, J. Chem. Phys., 126 (2007) 074311.

[144] D. Mathur, F.A. Rajgara, Nonadiabatic response of molecules to strong fields of picosecond, femtosecond, and subfemtosecond duration: An experimental study of the methane dication, J. Chem. Phys., 124 (2006) 194308. [145] K. Hoshina, H. Kawamura, M. Tsuge, M. Tamiya, M. Ishiguro, Metastable decomposition and hydrogen migration of ethane dication produced in an intense femtosecond near-infrared laser field, J. Chem. Phys., 134 (2011) 064324.

[146] C.Y. Wu, Q.Q. Liang, M. Liu, Y.K. Deng, Q.H. Gong, Ionization and dissociation of alkanes in few-cycle laser fields, Phys. Rev. A, 75 (2007) 043408.

[147] R. Ma, X. Li, H.Z. Ren, H. Yang, H.B. Jiang, Q.H. Gong, Intense femtosecond laser field-induced coulomb fragmentation of $\mathrm{C}_{2} \mathrm{H}_{4}$, Int. J. Mass Spectrom., 242 (2005) 43-48.

[148] J.X. Chen, R. Ma, X. Li, H.Z. Ren, H. Yang, Q.H. Gong, Coulomb explosion of propane in intense femtosecond laser fields, J. Phys. B, 37 (2004) 2501-2510.

[149] H.L. Xu, T. Okino, K. Yamanouchi, Tracing ultrafast hydrogen migration in allene in intense laser fields by triple-ion coincidence momentum imaging, J. Chem. Phys., 131 (2009) 151102.

[150] H.L. Xu, T. Okino, K. Nakai, K. Yamanouchi, S. Roither, X.H. Xie, D. Kartashov, M. Schoffler, A. Baltuska, M. Kitzler, Hydrogen migration and C-C bond breaking in 1,3-butadiene in intense laser fields studied by coincidence momentum imaging, Chem. Phys. Lett., 484 (2010) 119-123.

[151] H.L. Xu, T. Okino, K. Nakai, K. Yamanouchi, S. Roither, X.H. Xie, D. Kartashov, L. Zhang, A. Baltuska, M. Kitzler, Two-proton migration in 1,3-butadiene in intense laser fields, Phys. Chem. Chem. Phys., 12 (2010) 12939-12942.

[152] H.L. Xu, T. Okino, K. Yamanouchi, Ultrafast delocalization of hydrogen atoms in allene in intense laser fields, Appl. Phys. A, 104 (2011) 941-945.

[153] T. Okino, A. Watanabe, H.L. Xu, K. Yamanouchi, Two-body Coulomb explosion in methylacetylene in intense laser fields: double proton migration and proton/deuteron exchange, Phys. Chem. Chem. Phys., 14 (2012) $4230-4235$.

[154] T. Okino, A. Watanabe, H.L. Xu, K. Yamanouchi, Ultrafast hydrogen scrambling in methylacetylene and 
methyl- $d_{3}$-acetylene ions induced by intense laser fields, Phys. Chem. Chem. Phys., 14 (2012) 10640-10646.

[155] K. Hoshina, Y. Furukawa, T. Okino, K. Yamanouchi, Efficient ejection of $\mathrm{H}_{3}{ }^{+}$from hydrocarbon molecules induced by ultrashort intense lalifeser fields, J. Chem. Phys., 129 (2008) 104302.

[156] A. Hishikawa, A. Matsuda, M. Fushitani, E.J. Takahashi, Visualizing recurrently migrating hydrogen in acetylene dication by intense ultrashort laser pulses, Phys. Rev. Lett., 99 (2007) 258302.

[157] A. Matsuda, M. Fushitani, E.J. Takahashi, A. Hishikawa, Visualizing hydrogen atoms migrating in acetylene dication by time-resolved three-body and four-body Coulomb explosion imaging, Phys. Chem. Chem. Phys., 13 (2011) 8697-8704.

[158] M. Tanaka, M. Murakami, T. Yatsuhashi, N. Nakashima, Atomiclike ionization and fragmentation of a series of CH3-X (X : H, F, Cl, Br, I, and CN) by an intense femtosecond laser, J. Chem. Phys., 127 (2007).

[159] R. Kanya, T. Kudou, N. Schirmel, S. Miura, K.M. Weitzel, K. Hoshina, K. Yamanouchi, Hydrogen scrambling in ethane induced by intense laser fields: Statistical analysis of coincidence events, J. Chem. Phys., 136 (2012) 204309.

[160] A. Matsuda, M. Fushitani, R.A. Thomas, V. Zhaunerchyk, A. Hishikawa, Multiple explosion pathways of the deuterated benzene trication in 9-fs intense laser fields, J. Phys. Chem. A, 113 (2009) 2254-2260.

[161] T. Yatsuhashi, M. Murakami, N. Nakashima, Anisotropic bulletlike emission of terminal ethynyl fragment ions: Ionization of ethynylbenzene-d under intense femtosecond laser fields, J. Chem. Phys., 126 (2007) 194316. [162] A.N. Markevitch, D.A. Romanov, S.M. Smith, R.J. Levis, Probing strong-field electron-nuclear dynamics of polyatomic molecules using proton motion, Phys. Rev. A, 75 (2007) 053402.

[163] J. Zhang, Y. Yang, Z.P. Li, S.A. Zhang, Z.R. Sun, Dissociative photoionization of 1,2-dichloroethane in intense near-infrared femtosecond laser field, Chem. Phys. Lett., 667 (2017) 238-243.

[164] H. Wu, Y. Yang, S.Z. Sun, J. Zhang, L. Deng, S.A. Zhang, T.Q. Jia, Z.G. Wang, Z.R. Sun, Concerted elimination of $\mathrm{Br}_{2}{ }^{+}$resulting from the Coulomb explosion of 1,2-dibromoethane in an intense femtosecond laser field, Chem. Phys. Lett., 607 (2014) 70-74.

[165] C. Teng, H. Wu, J. Zhang, Y. Yang, T.Q. Jia, S.A. Zhang, Z.R. Sun, Dissociative ionization and Coulomb explosion of ethyl bromide under a near-infrared intense femtosecond laser field, Rsc Adv., 5 (2015) 37078-37084.

[166] T. Yatsuhashi, N. Nakashima, J. Azuma, Coulomb explosion of dichloroethene geometric isomers at 1 PW $\mathrm{cm}^{-2}$, J. Phys. Chem. A, 117 (2013) 1393-1399.

[167] S.Z. Sun, Y. Yang, J. Zhang, H. Wu, Y.T. Chen, S.A. Zhang, T.Q. Jia, Z.G. Wang, Z.R. Sun, Ejection of triatomic molecular ion $\mathrm{H}_{3}{ }^{+}$from methyl chloride in an intense femtosecond laser field, Chem. Phys. Lett., 581 (2013) 16-20.

[168] M.E. Corrales, G. Gitzinger, J. Gonzalez-Vazquez, V. Loriot, R. de Nalda, L. Banares, Velocity map imaging and theoretical study of the Coulomb explosion of $\mathrm{CH}_{3} \mathrm{I}$ under intense femtosecond IR pulses, J. Phys. Chem. A, 116 (2012) 2669-2677.

[169] A. du Plessis, C. Strydom, L. Botha, Comparative study of the dissociative Ionization of 1,1,1trichloroethane using nanosecond and femtosecond laser pulses, Int. J. Mol. Sci., 11 (2010) 1114-1140.

[170] X. Zhang, D.D. Zhang, H. Liu, H.F. Xu, M.X. Jin, D.J. Ding, Angular distributions of fragment ions in dissociative ionization of $\mathrm{CH}_{2} \mathrm{I}_{2}$ molecules in intense laser fields, J. Phys. B, 43 (2010) 025102.

[171] Y.M. Wang, S. Zhang, Z.R. Wei, B. Zhang, Velocity map imaging of dissociative ionization and coulomb 
explosion of $\mathrm{CH}_{3} \mathrm{I}$ induced by a femtosecond laser, J. Phys. Chem. A, 112 (2008) 3846-3851.

[172] Y. Yang, L.L. Fan, S.Z. Sun, J. Zhang, Y.T. Chen, S.A. Zhang, T.Q. Jia, Z.R. Sun, Dissociative double ionization of 1-bromo-2-chloroethane irradiated by an intense femtosecond laser field, J. Chem. Phys., 135 (2011) 064303.

[173] C. Kosmidis, P. Siozos, S. Kaziannis, L. Robson, K.W.D. Ledingham, P. McKenna, D.A. Jaroszynski, Interaction mechanism of some alkyl iodides with femtosecond laser pulses, J. Phys. Chem. A, 109 (2005) 12791285.

[174] H. Wu, S. Zhang, Y. Yang, S.Z. Sun, J. Zhang, L. Deng, T.Q. Jia, Z.G. Wang, Z.R. Sun, Coulomb explosion and dissociative ionization of 1,2-dibromoethane under an intense femtosecond laser field, Rsc Adv., 4 (2014) 45300-45305.

[175] Z.H. Liu, Y.Q. Wang, J.J. Ma, L. Wang, G.Z. He, Concerted elimination of $\mathrm{CH}_{2} \mathrm{I}_{2}$ and $\mathrm{CH}_{2} \mathrm{ICl}$ under intense femtosecond laser excitation, Chem. Phys. Lett., 383 (2004) 198-202.

[176] D.D. Zhang, S.Z. Luo, H.F. Xu, M.X. Jin, F.C. Liu, B. Yan, Z.G. Wang, H. Liu, D.W. Jiang, A. Eppink, W. Roeterdink, S. Stolte, D.J. Ding, Dissociative ionization and Coulomb explosion of $\mathrm{CH}_{3} \mathrm{I}$ in intense femto second laser fields, Eur. Phys. J. D, 71 (2017) 14

[177] M. Elshakre, Dissociative ionization of methanol in medium intense femtosecond laser field using time-offlight mass spectrometry, Radiat. Phys. Chem., 112 (2015) 49-55.

[178] J.X. Chen, R. Ma, H.Z. Ren, X. Li, H. Yang, Q.H. Gong, Femtosecond laser-induced dissociative ionization and Coulomb explosion of ethanol, Int. J. Mass Spectrom., 241 (2005) 25-29.

[179] H.L. Xu, C. Marceau, K. Nakai, T. Okino, S.L. Chin, K. Yamanouchi, Communication: Two stages of ultrafast hydrogen migration in methanol driven by intense laser fields, J. Chem. Phys., 133 (2010) 071103.

[180] H. Xu, T. Okino, T. Kudou, K. Yamanouchi, S. Roither, M. Kitzer, A. Batuska, S.L. Chin, Effect of laser parameters on ultrafast hydrogen migration in methanol studied by coincidence momentum imaging, J. Phys. Chem. A, 116 (2012) 2686-2690.

[181] K. Nakai, T. Kato, H. Kono, K. Yamanouchi, Communication: Long-lived neutral $\mathrm{H}_{2}$ in hydrogen migration within methanol dication, J. Chem. Phys., 139 (2013) 181103.

[182] P. Liu, T. Okino, Y. Furukawa, T. Ichikawa, R. Itakura, K. Hoshina, K. Yamanouchi, H. Nakano, Three-body sequential Coulomb explosions of $\mathrm{CH}_{3} \mathrm{OD}^{3+}$ induced by intense laser fields, Chem. Phys. Lett., 423 (2006) $187-$ 191.

[183] H. Tanaka, N. Nakashima, T. Yatsuhashi, Anisotropic Coulomb explosion of CO ligands in group 6 metal hexacarbonyls: $\mathrm{Cr}(\mathrm{CO})_{6}, \mathrm{Mo}(\mathrm{CO})_{6}, \mathrm{~W}(\mathrm{CO})_{6}$, J. Phys. Chem. A, 120 (2016) 6917-6928.

[184] T. Yatsuhashi, E. Murakami, N. Nakashima, Fez+ $(z=1-6)$ generation from ferrocene, Phys. Chem. Chem. Phys., 13 (2011) 4234-4238.

[185] C. Wang, B. Wang, M. Okunishi, W.G. Roeterdink, D. Ding, R. Zhu, G. Prumper, K. Shimada, K. Ueda, Ionion coincidence imaging of dissociative ionization dynamics of formic acid in intense laser fields, Chem. Phys., 430 (2014) 40-46.

[186] T. Yatsuhashi, T. Obayashi, M. Tanaka, M. Murakami, N. Nakashima, Femtosecond laser ionization of organic amines with very low ionization potentials: Relatively small suppressed ionization features, J. Phys. Chem. A, 110 (2006) 7763-7771. 
[187] T. Yatsuhashi, N. Nakashima, Dissociation and multiply charged silicon ejection in high abundance from hexamethyldisilane, J. Phys. Chem. A, 114 (2010) 11890-11895.

[188] S.G. Sayres, M.W. Ross, A.W. Castleman, Ultrafast ionization and fragmentation of molecular silane, Phys. Rev. A, 82 (2010) 033424.

[189] Y. Boran, A.A. Kolomenskii, M. Sayrac, N. Kaya, H.A. Schuessler, J. Strohaber, Dissociative ionization of acetonitrile in intense femtosecond laser fields, J. Phys. B, 50 (2017) 135003.

[190] C. Mullen, M.J. Coggiola, H. Oser, Femtosecond laser photoionization time-of-flight mass spectrometry of nitro-aromatic explosives and explosives related compounds, J. Am. Soc. Mass. Spectrom., 20 (2009) 419-429.

[191] Y. Albeck, D.M. Kandhasamy, D. Strasser, Multiple Detachment of the $\mathrm{SF}_{6}{ }^{-}$molecular anion with shaped intense laser pulses, J. Phys. Chem. A, 118 (2014) 388-395.

[192] H.Z. Ren, R. Ma, X. Li, J.X. Chen, H. Yang, Q.H. Gong, Multielectron dissociative ionization of SF 6 in an intense femtosecond laser field, Int. J. Mass Spectrom., 235 (2004) 117-122.

[193] W.C. Wiley, I.H. Mclaren, Time-of-flight mass spectrometer with improved resolution, Rev. Sci. Instrum., 26 (1955) 1150-1157.

[194] A. Hishikawa, A. Iwamae, K. Hoshina, M. Kono, K. Yamanouchi, Mass-resolved two-dimensional momentum imaging of the Coulomb explosion of $\mathrm{N}_{2}$ and $\mathrm{SO}_{2}$ in an intense laser field, Chem. Phys. Lett., 282 (1998) 283-291.

[195] A. Hishikawa, A. Iwamae, K. Yamanouchi, Ultrafast deformation of the geometrical structure of $\mathrm{CO}_{2}$ induced in intense laser fields, Phys. Rev. Lett., 83 (1999) 1127-1130.

[196] A. Hishikawa, A. Iwamae, K. Hoshina, M. Kono, K. Yamanouchi, Coulomb explosion dynamics of $\mathrm{N}_{2}$ in intense laser field by mass-resolved momentum imaging, Chem. Phys., 231 (1998) 315-329.

[197] A. Hishikawa, S.L. Liu, A. Iwasaki, K. Yamanouchi, Light-induced multiple electronic-state coupling of $\mathrm{O}_{2}^{+}$ in intense laser fields, J. Chem. Phys., 114 (2001) 9856-9862..

[198] L.J. Frasinski, Covariance mapping techniques, J. Phys. B, 49 (2016) 152004.

[199] L.J. Frasinski, K. Codling, P.A. Hatherly, Covariance mapping: a correlation method applied to multiphoton multiple ionization, Science, 246 (1989) 1029-1031.

[200] C. Cornaggia, Statistical analysis of fragmentation channels of small multicharged molecular ions, J. Phys. B, 45 (2012) 085602.

[201] H. Hasegawa, A. Hishikawa, K. Yamanouchi, Coincidence imaging of Coulomb explosion of CS2 in intense laser fields, Chem. Phys. Lett., 349 (2001) 57-63.

[202] A.T.J.B. Eppink, D.H. Parker, Velocity map imaging of ions and electrons using electrostatic lenses: Application in photoelectron and photofragment ion imaging of molecular oxygen, Rev. Sci. Instrum., 68 (1997) 3477-3484.

[203] J.D. Pickering, K. Amini, M. Brouard, M. Burt, I.J. Bush, L. Christensen, A. Lauer, J.H. Nielsen, C.S. Slater, H. Stapelfeldt, Communication: Three-fold covariance imaging of laser-induced Coulomb explosions, J. Chem. Phys., 144 (2016) 161105.

[204] C.S. Slater, S. Blake, M. Brouard, A. Lauer, C. Vallance, J.J. John, R. Turchetta, A. Nomerotski, L. Christensen, J.H. Nielsen, M.P. Johansson, H. Stapelfeldt, Covariance imaging experiments using a pixel-imaging mass-spectrometry camera, Phys. Rev. A, 89 (2014) 011401. 
[205] J.L. Hansen, J.H. Nielsen, C.B. Madsen, A.T. Lindhardt, M.P. Johansson, T. Skrydstrup, L.B. Madsen, H. Stapelfeldt, Control and femtosecond time-resolved imaging of torsion in a chiral molecule, J. Chem. Phys., 136 (2012) 20431.

[206] D.A. Card, E.S. Wisniewski, D.E. Folmer, A.W. Castleman, Dynamics of Coulomb explosion and kinetic energy release in clusters of heterocyclic compounds, J. Chem. Phys., 116 (2002) 3554-3567.

[207] D.A. Card, D.E. Folmer, S. Kooi, S. Sato, A.W. Castleman, The interaction of titanium-carbon cluster fragments, Eur. Phys. J. D, 9 (1999) 195-200.

[208] D.A. Card, D.E. Folmer, S. Sato, S.A. Buzza, A.W. Castleman, Covariance mapping of ammonia clusters: Evidence of the connectiveness of clusters with Coulombic explosion, J. Phys. Chem. A, 101 (1997) 3417-3423.

[209] D.A. Card, E.S. Wisniewski, D.E. Folmer, A.W. Castleman, The relationship between covariance and anticovariance mapping, Int. J. Mass Spectrom., 223 (2003) 355-363.

[210] P. Hering, C. Cornaggia, Coulomb explosion of $\mathrm{N}_{2}$ and $\mathrm{CO}_{2}$ using linearly and circularly polarized femtosecond laser pulses, Phys. Rev. A, 59 (1999) 2836-2843.

[211] V. Zhaunerchyk, M. Kaminska, M. Mucke, R.J. Squibb, J.H.D. Eland, M.N. Piancastelli, L.J. Frasinski, J. Grilj, M. Koch, B.K. McFarland, E. Sistrunk, M. Guhr, R.N. Coffee, C. Bostedt, J.D. Bozek, P. Salen, P.V.D. Meulen, P. Linusson, R.D. Thomas, M. Larsson, L. Foucar, J. Ullrich, K. Motomura, S. Mondal, K. Ueda, R. Richter, K.C. Prince, O. Takahashi, T. Osipov, L. Fang, B.F. Murphy, N. Berrah, R. Feifel, Disentangling formation of multiple-core holes in aminophenol molecules exposed to bright X-FEL radiation, J. Phys. B, 48 (2015) 244003. [212] M. Mucke, V. Zhaunerchyk, L.J. Frasinski, R.J. Squibb, M. Siano, J.H.D. Eland, P. Linusson, P. Salen, P. von der Meulen, R.D. Thomas, M. Larsson, L. Foucar, J. Ullrich, K. Motomura, S. Mondal, K. Ueda, T. Osipov, L. Fang, B.F. Murphy, N. Berrah, C. Bostedt, J.D. Bozek, S. Schorb, M. Messerschmidt, J.M. Glownia, J.P. Cryan, R.N. Coffee, O. Takahashi, S. Wada, M.N. Piancastelli, R. Richter, K.C. Prince, R. Feifel, Covariance mapping of two-photon double core hole states in $\mathrm{C}_{2} \mathrm{H}_{2}$ and $\mathrm{C}_{2} \mathrm{H}_{6}$ produced by an X-ray free electron laser, New. J. Phys., 17 (2015) 073002

[213] B. Dick, Inverting ion images without Abel inversion: maximum entropy reconstruction of velocity maps, Phys. Chem. Chem. Phys., 16 (2014) 570-580.

[214] A.I. Chichinin, K.H. Gericke, S. Kauczok, C. Maul, Imaging chemical reactions-3D velocity mapping, Int. Rev. Phys. Chem., 28 (2009) 607-680.

[215] C.R. Gebhardt, T.P. Rakitzis, P.C. Samartzis, V. Ladopoulos, T.N. Kitsopoulos, Slice imaging: A new approach to ion imaging and velocity mapping, Rev. Sci. Instrum., 72 (2001) 3848-3853.

[216] M.N.R. Ashfold, N.H. Nahler, A.J. Orr-Ewing, O.P.J. Vieuxmaire, R.L. Toomes, T.N. Kitsopoulos, I.A. Garcia, D.A. Chestakov, S.M. Wu, D.H. Parker, Imaging the dynamics of gas phase reactions, Phys. Chem. Chem. Phys., 8 (2006) 26-53.

[217] T. Suzuki, B.J. Whitaker, Non-adiabatic effects in chemistry revealed by time-resolved charged-particle imaging, Int. Rev. Phys. Chem., 20 (2001) 313-356.

[218] C. Lee, Y.C. Lin, S.H. Lee, Y.Y. Lee, C.M. Tseng, Y.T. Lee, C.K. Ni, Advantage of spatial map ion imaging in the study of large molecule photodissociation, J. Chem. Phys., 147 (2017) 013904.

[219] S.A. Aseyev, Y. Ni, L.J. Frasinski, H.G. Muller, M.J.J. Vrakking, Attosecond angle-resolved photoelectron spectroscopy, Phys. Rev. Lett., 91 (2003) 223902. 
[220] T. Endo, A. Matsuda, M. Fushitani, T. Yasuike, O.I. Tolstikhin, T. Morishita, A. Hishikawa, Imaging electronic excitation of NO by ultrafast laser tunneling ionization, Phys. Rev. Lett., 116 (2016) 163002.

[221] T. Endo, H. Fujise, A. Matsuda, M. Fushitani, H. Kono, A. Hishikawa, Coincidence momentum imaging of asymmetric Coulomb explosion of $\mathrm{CO}_{2}$ in phase-locked two-color intense laser fields, J. Electron. Spectrosc. Relat. Phenom., 207 (2016) 50-54.

[222] T. Endo, H. Fujise, Y. Kawachi, A. Ishihara, A. Matsuda, M. Fushitani, H. Kono, A. Hishikawa, Selective bond breaking of $\mathrm{CO}_{2}$ in phase-locked two-color intense laser fields: laser field intensity dependence, Phys. Chem. Chem. Phys., 19 (2017) 3550-3556.

[223] H. Ibrahim, B. Wales, S. Beaulieu, B.E. Schmidt, N. Thire, E.P. Fowe, E. Bisson, C.T. Hebeisen, V. Wanie, M. Giguere, J.C. Kieffer, M. Spanner, A.D. Bandrauk, J. Sanderson, M.S. Schuurman, F. Legare, Tabletop imaging of structural evolutions in chemical reactions demonstrated for the acetylene cation, Nat. Commun., 5 (2014) 4422. [224] C. Burger, N.G. Kling, R. Siemering, A.S. Alnaser, B. Bergues, A.M. Azzeer, R. Moshammer, R. de VivieRiedle, M. Kubel, M.F. Kling, Visualization of bond rearrangements in acetylene using near single-cycle laser pulses, Faraday Discuss., 194 (2016) 495-508.

[225] R. Dorner, V. Mergel, O. Jagutzki, L. Spielberger, J. Ullrich, R. Moshammer, H. Schmidt-Bocking, Cold Target Recoil Ion Momentum Spectroscopy: a 'momentum microscope' to view atomic collision dynamics, Phys. Rep., 330 (2000) 95-192.

[226] J. Ullrich, R. Moshammer, A. Dorn, R. Dorner, L.P.H. Schmidt, H. Schmidt-Bocking, Recoil-ion and electron momentum spectroscopy: reaction-microscopes, Rep. Prog. Phys., 66 (2003) 1463-1545.

[227] M. Comstock, V. Senekerimyan, M. Dantus, Ultrafast laser induced molecular alignment and deformation: Experimental evidence from neutral molecules and from fragment ions, J. Phys. Chem. A, 107 (2003) 8271-8281. [228] H. Sakai, C.P. Safvan, J.J. Larsen, K.M. Hilligsoe, K. Hald, H. Stapelfeldt, Controlling the alignment of neutral molecules by a strong laser field, J. Chem. Phys., 110 (1999) 10235-10238.

[229] U. Buck, M. Farnik, Oriented xenon hydride molecules in the gas phase, Int. Rev. Phys. Chem., 25 (2006) 583-612.

[230] H. Sakai, S. Minemoto, H. Nanjo, H. Tanji, T. Suzuki, Controlling the orientation of polar molecules with combined electrostatic and pulsed, nonresonant laser fields, Phys. Rev. Lett., 90 (2003) 083001.

[231] H. Tanji, S. Minemoto, H. Sakai, Three-dimensional molecular orientation with combined electrostatic and elliptically polarized laser fields, Phys. Rev. A, 72 (2005) 63401.

[232] L. Holmegaard, J.H. Nielsen, I. Nevo, H. Stapelfeldt, F. Filsinger, J. Kupper, G. Meijer, Laser-Induced Alignment and Orientation of Quantum-State-Selected Large Molecules, Phys. Rev. Lett., 102 (2009) 023001. [233] I. Nevo, L. Holmegaard, J.H. Nielsen, J.L. Hansen, H. Stapelfeldt, F. Filsinger, G. Meijer, J. Kupper, Laserinduced 3D alignment and orientation of quantum state-selected molecules, Phys. Chem. Chem. Phys., 11 (2009) 9912-9918.

[234] K. Amini, R. Boll, A. Lauer, M. Burt, J.W.L. Lee, L. Christensen, F. Brausse, T. Mullins, E. Savelyev, U. Ablikim, N. Berrah, C. Bomme, S. Dusterer, B. Erk, H. Hoppner, P. Johnsson, T. Kierspel, F. Krecinic, J. Kupper, M. Muller, E. Muller, H. Redlin, A. Rouzee, N. Schirmel, J. Thogersen, S. Techert, S. Toleikis, R. Treusch, S. Trippel, A. Ulmer, J. Wiese, C. Vallance, A. Rudenko, H. Stapelfeldt, M. Brouard, D. Rolles, Alignment, orientation, and Coulomb explosion of difluoroiodobenzene studied with the pixel imaging mass spectrometry 
(PImMS) camera, J. Chem. Phys., 147 (2017) 013933.

[235] K. Oda, M. Hita, S. Minemoto, H. Sakai, All-optical molecular orientation, Phys. Rev. Lett., 104 (2010) 213901 .

[236] L. Christensen, L. Christiansen, B. Shepperson, H. Stapelfeldt, Deconvoluting nonaxial recoil in Coulomb explosion measurements of molecular axis alignment, Phys. Rev. A, 94 (2016) 023410.

[237] U. Ablikim, C. Bomme, H. Xiong, E. Savelyev, R. Obaid, B. Kaderiya, S. Augustin, K. Schnorr, I. Dumitriu, T. Osipov, R. Bilodeau, D. Kilcoyne, V. Kumarappan, A. Rudenko, N. Berrah, D. Rolles, Identification of absolute geometries of cis and trans molecular isomers by Coulomb Explosion Imaging, Sci.Rep., 6 (2016) 38202.

[238] U. Ablikim, C. Bomme, E. Savelyev, H. Xiong, R. Kushawaha, R. Boll, K. Amini, T. Osipov, D. Kilcoyne, A. Rudenko, N. Berrah, D. Rolles, Isomer-dependent fragmentation dynamics of inner-shell photoionized difluoroiodobenzene, Phys. Chem. Chem. Phys., 21 (2017) 13419-13431.

[239] C. Lux, M. Wollenhaupt, T. Bolze, Q.Q. Liang, J. Kohler, C. Sarpe, T. Baumert, Circular dichroism in the photoelectron angular distributions of camphor and fenchone from multiphoton ionization with femtosecond laser pulses, Angew. Chem. Int. Ed., 51 (2012) 5001-5005.

[240] D. Patterson, M. Schnell, J.M. Doyle, Enantiomer-specific detection of chiral molecules via microwave spectroscopy, Nature, 497 (2013) 475-478.

[241] D. Patterson, M. Schnell, New studies on molecular chirality in the gas phase: enantiomer differentiation and determination of enantiomeric excess, Phys. Chem. Chem. Phys., 16 (2014) 11114-11123.

[242] T. Kitamura, T. Nishide, H. Shiromaru, Y. Achiba, N. Kobayashi, Direct observation of "dynamic" chirality by Coulomb explosion imaging, J. Chem. Phys., 115 (2001) 5-6.

[243] M. Pitzer, M. Kunitski, A.S. Johnson, T. Jahnke, H. Sann, F. Sturm, L.P.H. Schmidt, H. Schmidt-Bocking, R. Dorner, J. Stohner, J. Kiedrowski, M. Reggelin, S. Marquardt, A. Schiesser, R. Berger, M.S. Schoffler, Direct determination of absolute molecular stereochemistry in gas phase by Coulomb explosion imaging, Science, 341 (2013) 1096-1100.

[244] M. Pitzer, G. Kastirke, M. Kunitski, T. Jahnke, T. Bauer, C. Goihl, F. Trinter, C. Schober, K. Henrichs, J. Becht, S. Zeller, H. Gassert, M. Waitz, A. Kuhlins, H. Sann, F. Sturm, F. Wiegandt, R. Wallauer, L.P.H. Schmidt, A.S. Johnson, M. Mazenauer, B. Spenger, S. Marquardt, S. Marquardt, H. Schmidt-Bocking, J. Stohner, R. Dorner, M. Schoffler, R. Berger, Absolute configuration from different multifragmentation pathways in light-induced Coulomb explosion imaging, ChemPhysChem, 17 (2016) 2465-2472.

[245] M. Pitzer, G. Kastirke, P. Burzynski, M. Weller, D. Metz, J. Neff, M. Waitz, F. Trinter, L.P.H. Schmidt, J.B. Williams, T. Jahnke, H. Schmidt-Bocking, R. Berger, R. Dorner, M. Schoffler, Stereochemical configuration and selective excitation of the chiral molecule halothane, J. Phys. B, 49 (2016) 234001.

[246] R. Noyori, H. Takaya, BINAP: an efficient chiral element for asymmetric catalysis, Acc. Chem. Res., 23 (1990) 345-350.

[247] L. Christensen, J.H. Nielsen, C.S. Slater, A. Lauer, M. Brouard, H. Stapelfeldt, Using laser-induced Coulomb explosion of aligned chiral molecules to determine their absolute configuration, Phys. Rev. A, 92 (2015) 033411. [248] Y.M. Fan, C.Y. Wu, X.G. Xie, P. Wang, X.Q. Zhong, Y. Shao, X.F. Sun, Y.Q. Liu, Q.H. Gong, Three-body fragmentation dynamics of carbon-dioxide dimers induced by intense femtosecond laser pulses, Chem. Phys. Lett., 653 (2016) 108-111. 
[249] M. Amada, Y. Sato, M. Tsuge, K. Hoshina, Near-infrared femtosecond laser ionization of the acetic acid dimer, Chem. Phys. Lett., 624 (2015) 24-28.

[250] K. Hoshina, H. Hagihara, M. Tsuge, Double ionization and Coulomb explosion of the formic acid dimer by intense near-infrared femtosecond laser pulses, J. Phys. Chem. A, 116 (2012) 826-831.

[251] Z.P. Wang, P.M. Dinh, P.G. Reinhard, E. Suraud, Ultrafast nonadiabatic dynamics of a water dimer in femtosecond laser pulses, Laser Phys., 24 (2014) 106004.

[252] J. Wu, M. Kunitski, L.P.H. Schmidt, T. Jahnke, R. Dorner, Structures of N2Ar, O2Ar, and O2Xe dimers studied by Coulomb explosion imaging, J. Chem. Phys., 137 (2012) 104308.

[253] C.Y. Wu, C. Wu, D. Song, H.M. Su, X.G. Xie, M. Li, Y.K. Deng, Y.Q. Liu, Q.H. Gong, Communication: Determining the structure of the $\mathrm{N}_{2} \mathrm{Ar}$ van der Waals complex with laser-based channel-selected Coulomb explosion, J. Chem. Phys., 140 (2014) 141101.

[254] X. Gong, M. Kunitski, L.P.H. Schmid T. Jahnke, A. Czasch, R. Dorner, J. Wu, Simultaneous probing of geometry and electronic orbital of ArCO by Coulomb-explosion imaging and angle-dependent tunneling rates, Phys. Rev. A, 88 (2013) 013422.

[255] T. Fennel, K.H. Meiwes-Broer, J. Tiggesbaumker, P.G. Reinhard, P.M. Dinh, E. Suraud, Laser-driven nonlinear cluster dynamics, Rev. Mod. Phys., 82 (2010) 1793-1842.

[256] S.R. Krishnan, R. Gopal, R. Rajeev, J. Jha, V. Sharma, M. Mudrich, R. Moshammer, M. Krishnamurthy, Photoionization of clusters in intense few-cycle near infrared femtosecond pulses, Phys. Chem. Chem. Phys., 16 (2014) 8721-8730.

[257] T. Ditmire, J. Zweiback, V.P. Yanovsky, T.E. Cowan, G. Hays, K.B. Wharton, Nuclear fusion from explosions of femtosecond laser-heated deuterium clusters, Nature, 398 (1999) 489-492.

[258] M.W. Ross, A.W. Castleman, Strong-field ionization and Coulomb explosion of small neodymium and europium oxide clusters, Chem. Phys. Lett., 565 (2013) 22-27.

[259] M.W. Ross, A.W. Castleman, Ionization and Coulomb explosion of small uranium oxide clusters, J. Phys. B, 45 (2012) 205102.

[260] M.W. Ross, A.W. Castleman, Femtosecond ionization and Coulomb explosion of small transition metal carbide clusters, Chem. Phys. Lett., 547 (2012) 13-20.

[261] D.E. Blumling, S.G. Sayres, A.W. Castleman, Strong-field ionization and dissociation studies of small early transition metal oxide clusters, Int. J. Mass Spectrom., 300 (2011) 74-80.

[262] M.W. Ross, A.W. Castleman, Extreme ionization leading to Coulomb explosion of small palladium and zirconium oxide clusters and reactivity with carbon monoxide, J. Phys. Chem. A, 117 (2013) 1030-1034.

[263] D.E. Blumling, S.G. Sayres, M.W. Ross, A.W. Castleman, Strong-field ionization of small niobium and tantalum clusters, Int. J. Mass Spectrom., 333 (2013) 55-58.

[264] M.W. Ross, A.W. Castleman, Ionization and Coulomb explosion of small group 10 transition metal oxide clusters in strong light fields, J. Chem. Phys., 137 (2012) 084307.

[265] M.W. Ross, A.W. Castleman, Ultrafast ionization and subsequent coulomb explosion of zirconium oxide and tungsten carbide "superatomic" cluster species and comparison to group 10 metals, New J. Chem., 36 (2012) 22532259.

[266] D.E. Blumling, S.G. Sayres, A.W. Castleman, Strong-field ionization and dissociation studies on small early 
transition metal carbide clusters via time-of-flight mass spectrometry, J. Phys. Chem. A, 115 (2011) 5038-5043. [267] M. Smits, C.A. de Lange, A. Stolow, D.M. Rayner, Absolute ionization rates of multielectron transition metal atoms in strong infrared laser fields, Phys. Rev. Lett., 93 (2004) 213003.

[268] M. Smits, C.A. de Lange, A. Stolow, D.M. Rayner, Dynamic polarization in the strong-field ionization of small metal clusters, Phys. Rev. Lett., 93 (2004) 203402.

[269] E.M. Snyder, S. Wei, J. Purnell, S.A. Buzza, A.W. Castleman, Femtosecond laser-induced Coulomb explosion of ammonia clusters, Chem. Phys. Lett., 248 (1996) 1-7.

[270] L. Poth, Q. Zhong, J.V. Ford, S.M. Hurley, A.W. Castleman, Charge stripping effects from highly charged iodine ions formed from Coulomb explosion of $\mathrm{CH}_{3}$ I clusters, Chem. Phys., 239 (1998) 309-315.

[271] J.V. Ford, Q. Zhong, L. Poth, A.W. Castleman, Femtosecond laser interactions with methyl iodide clusters. I. Coulomb explosion at 795 nm, J. Chem. Phys., 110 (1999) 6257-6267.

[272] J.V. Ford, L. Poth, Q. Zhong, A.W. Castleman, Femtosecond laser interactions with methyl iodide clusters.

2. Coulomb explosion at $397 \mathrm{~nm}$, Int. J. Mass Spectrom., 192 (1999) 327-345.

[273] E.S. Wisniewski, A.W. Castleman, Comparison of methyl and hydroxyl protons generated in a Coulomb explosion event: application of a time-of-flight gating technique to methanol clusters, Int. J. Mass Spectrom., 227 (2003) 577-585.

[274] S. Li, Z.L. Zhou, Y. Tian, H.Y. Lu, W.T. Wang, J.J. Ju, H.Y. Li, Y. Xu, Y.X. Leng, G.Q. Ni, C. Wang, J.S. Liu, Energetic proton generation from intense Coulomb explosion of large-size ethane clusters, Phys. Plasmas., 20 (2013) 043109.

[275] G. Karras, C. Kosmidis, Multi-electron dissociative ionization of clusters under picosecond and femtosecond laser irradiation: the case of alkyl-halide clusters, Phys. Chem. Chem. Phys., 14 (2012) 12147-12156.

[276] S.G. Sayres, M.W. Ross, A.W. Castleman, Influence of clustering and molecular orbital shapes on the ionization enhancement in ammonia, Phys. Chem. Chem. Phys., 13 (2011) 12231-12239.

[277] J. Wu, A. Vredenborg, L.P.H. Schmidt, T. Jahnke, A. Czasch, R. Dorner, Comparison of dissociative ionization of $\mathrm{H}_{2}, \mathrm{~N}_{2}, \mathrm{Ar}_{2}$, and $\mathrm{CO}$ by elliptically polarized two-color pulses, Phys. Rev. A, 87 (2013) 023406.

[278] R. Itakura, K. Yamanouchi, T. Yasuike, K. Someda, Formation of $\left.\left[\mathrm{C}_{5} \mathrm{H}_{6}\left(\mathrm{NH}_{3}\right)_{2}\right)\right]^{+}$and $\left[\mathrm{NH}_{4}\left(\mathrm{NH}_{3}\right)_{m}\right]^{+}(m=1-$ 3) from size-selected aniline-ammonia cluster cations in intense laser fields, Chem. Phys. Lett., 396 (2004) 208212.

[279] R. Itakura, T. Asano, K. Yamanouchi, Suppression of decomposition of aniline cation in intense laser fields by cluster formation with $\mathrm{NH}_{3}$ and $\mathrm{H}_{2} \mathrm{O}$, J. Photochem. Photobiol., A, 158 (2003) 77-81.

[280] T. Ebata, T. Watanabe, N. Mikami, Evidence for the cyclic form of phenol trimer: vibrational spectroscopy of the OH stretching vibrations of jet-cooled phenol dimer and trimer, J. Phys. Chem., 99 (1995) 5761-5764.

[281] K. Sakota, N. Inoue, Y. Komoto, H. Sekiya, Cooperative triple-proton/hydrogen atom relay in 7azaindole $\left(\mathrm{CH}_{3} \mathrm{OH}\right)_{2}$ in the gas phase: Remarkable change in the reaction mechanism from vibrational-mode specific to statistical fashion with increasing internal energy, J. Phys. Chem. A, 111 (2007) 4596-4603.

[282] K. Sakota, C. Jouvet, C. Dedonder, M. Fujii, H. Sekiya, Excited-state triple-proton transfer in 7azaindole $\left(\mathrm{H}_{2} \mathrm{O}\right)_{2}$ and reaction path studied by electronic spectroscopy in the gas phase and quantum chemical calculations, J. Phys. Chem. A, 114 (2010) 11161-11166. 
[283] N. Sato, Y. Saito, H. Shinohara, Threshold ionization energy of $\mathrm{C}_{60}$ in the solid-state, Chem. Phys., 162 (1992) 433-438.

[284] M. Henyk, D. Wolfframm, J. Reif, Ultra short laser pulse induced charged particle emission from wide bandgap crystals, Appl. Surf. Sci., 168 (2000) 263-266.

[285] R. Stoian, D. Ashkenasi, A. Rosenfeld, E.E.B. Campbell, Coulomb explosion in ultrashort pulsed laser ablation of $\mathrm{Al}_{2} \mathrm{O}_{3}$, Phys. Rev. B, 62 (2000) 13167-13173.

[286] W.G. Roeterdink, L.B.F. Juurlink, O.P.H. Vaughan, J.D. Diez, M. Bonn, A.W. Kleyn, Coulomb explosion in femtosecond laser ablation of Si(111), Appl. Phys. Lett., 82 (2003) 4190-4192.

[287] H. Dachraoui, W. Husinsky, Fast electronic and thermal processes in femtosecond laser ablation of Au, Appl. Phys. Lett., 89 (2006) 104102.

[288] H. Dachraoui, W. Husinsky, G. Betz, Ultra-short laser ablation of metals and semiconductors: evidence of ultra-fast Coulomb explosion, Appl. Phys. A, 83 (2006) 333-336.

[289] R. Stoian, A. Rosenfeld, D. Ashkenasi, I.V. Hertel, N.M. Bulgakova, E.E.B. Campbell, Surface charging and impulsive ion ejection during ultrashort pulsed laser ablation, Phys. Rev. Lett., 88 (2002) 097603.

[290] N.M. Bulgakova, R. Stoian, A. Rosenfeld, Laser-induced modification of transparent crystals and glasses, Quantum Electron., 40 (2010) 966-985.

[291] F. Liang, R. Vallee, Femtosecond laser-induced ultra-fine nanostructures on silicon surface, Opt. Mater. Express., 6 (2016) 3330-3338.

[292] S. Bashir, M.S. Rafique, W. Husinsky, Identification of ultra-fast electronic and thermal processes during femtosecond laser ablation of Si, Appl. Phys. A, 109 (2012) 421-429.

[293] S. Bashir, M.S. Rafique, W. Husinsky, Identification of non-thermal and thermal processes in femtosecond laser-ablated aluminum, Radiat Eff. Defects Solids, 168 (2013) 902-911.

[294] S. Bashir, M.S. Rafique, W. Husinsky, Surface topography (nano-sized hillocks) and particle emission of metals, dielectrics and semiconductors during ultra-short-laser ablation: Towards a coherent understanding of relevant processes, Appl. Surf. Sci., 255 (2009) 8372-8376.

[295] M. Lancry, B. Poumellec, J. Canning, K. Cook, J.C. Poulin, F. Brisset, Ultrafast nanoporous silica formation driven by femtosecond laser irradiation, Laser Photonics Rev., 7 (2013) 953-962.

[296] L.T. Qi, K. Nishii, M. Yasui, H. Aoki, Y. Namba, Femtosecond laser ablation of sapphire on different crystallographic facet planes by single and multiple laser pulses irradiation, Opt. Laser Eng., 48 (2010) 1000-1007.

[297] J. Hernandez-Rueda, J. Siegel, M. Galvan-Sosa, A.R. de la Cruz, M. Garcia-Lechuga, J. Solis, Controlling ablation mechanisms in sapphire by tuning the temporal shape of femtosecond laser pulses, J. Opt. Soc. Am. B, 32 (2015) 150-156.

[298] G. Geoffroy, G. Duchateau, N. Fedorov, P. Martin, S. Guizard, Influence of electron Coulomb explosion on photoelectron spectra of dielectrics irradiated by femtosecond laser pulses, Laser Phys., 24 (2014) 086101.

[299] J. Hernandez-Rueda, D. Puerto, J. Siegel, M. Galvan-Sosa, J. Solis, Plasma dynamics and structural modifications induced by femtosecond laser pulses in quartz, Appl. Surf. Sci., 258 (2012) 9389-9393.

[300] S. Sakabe, M. Hashida, S. Tokita, S. Namba, K. Okamuro, Mechanism for self-formation of periodic grating structures on a metal surface by a femtosecond laser pulse, Phys. Rev. B, 79 (2009) 033409.

[301] M. Hashida, S. Namba, K. Okamuro, S. Tokita, S. Sakabe, Ion emission from a metal surface through a 
multiphoton process and optical field ionization, Phys. Rev. B, 81 (2010) 115442.

[302] Y. Miyasaka, M. Hashida, Y. Ikuta, K. Otani, S. Tokita, S. Sakabe, Nonthermal emission of energetic ions from a metal surface irradiated by extremely low-fluence femtosecond laser pulses, Phys. Rev. B, 86 (2012) 07543. [303] V.E. Gruzdev, X. Li, V.L. Komolov, S.G. Przhibel'skii, D.S. Smirnov, Modification of a dielectric surface when laser-induced Coulomb explosion of nanoparticles occurs, J. Opt. Technol., 78 (2011) 498-504.

[304] V.L. Komolov, V.E. Gruzdev, S.G. Przhibelskii, D.S. Smirnov, Dynamics of laser-induced damage of spherical nanoparticles by high-intensity ultrashort laser pulses, Opt. Eng., 51 (2012) 121816.

[305] C.T. Hebeisen, G. Sciaini, M. Harb, R. Ernstorfer, S.G. Kruglik, R.J.D. Miller, Direct visualization of charge distributions during femtosecond laser ablation of a Si(100) surface, Phys. Rev. B, 78 (2008) 081403.

[306] J.J. Li, X. Wang, Z.Y. Chen, J. Zhou, S.S. Mao, J.M. Cao, Real-time probing of ultrafast residual charge dynamics, Appl. Phys. Lett., 98 (2011) 011501.

[307] S. Inoue, S. Tokita, K. Otani, M. Hashida, S. Sakabe, Femtosecond electron deflectometry for measuring transient fields generated by laser-accelerated fast electrons, Appl. Phys. Lett., 99 (2011) 031501.

[308] C. Cirelli, M. Hengsberger, A. Dolocan, H. Over, J. Osterwalder, T. Greber, Direct observation of space charge dynamics by picosecond low-energy electron scattering, Epl, 85 (2009) 17010.

[309] S. Inoue, S. Tokita, M. Hashida, S. Sakabe, Transient changes in electric fields induced by interaction of ultraintense laser pulses with insulator and metal foils: Sustainable fields spanning several millimeters, Phys. Rev. E, 91 (2015) 043101.

[310] S. Inoue, S. Tokita, K. Otani, M. Hashida, M. Hata, H. Sakagami, T. Taguchi, S. Sakabe, Autocorrelation measurement of fast electron pulses emitted through the interaction of femtosecond laser pulses with a solid target, Phys. Rev. Lett., 109 (2012) 185001.

[311] S. Tao, B.X. Wu, Early-stage effects of residual charges in a metal target on emitted electrons induced by femtosecond laser-metal interactions, Phys. Lett. A, 381 (2017) 404-407.

[312] S. Tao, B.X. Wu, The effect of emitted electrons during femtosecond laser-metal interactions: A physical explanation for coulomb explosion in metals, Appl. Surf. Sci., 298 (2014) 90-94.

[313] S.C. Li, S.Y. Li, F.J. Zhang, D. Tian, H. Li, D.L. Liu, Y.F. Jiang, A.M. Chen, M.X. Jin, Possible evidence of Coulomb explosion in the femtosecond laser ablation of metal at low laser fluence, Appl. Surf. Sci., 355 (2015) 681-685.

[314] M. Djouder, O. Lamrous, M.D. Mitiche, T.E. Itina, M. Zemirli, Electromagnetic particle-in-cell (PIC) method for modeling the formation of metal surface structures induced by femtosecond laser radiation, Appl. Surf. Sci., 280 (2013) 711-714.

[315] E.B. Yakovlev, O.N. Sergaeva, V.V. Svirina, The effect of electron emission on the heating of metals by femtosecond laser pulses, J. Opt. Technol., 78 (2011) 487-490.

[316] E. Axente, S. Noel, J. Hermann, M. Sentis, I.N. Mihailescu, Subpicosecond laser ablation of copper and fused silica: Initiation threshold and plasma expansion, Appl. Surf. Sci., 255 (2009) 9734-9737.

[317] M. Hashida, A.F. Semerok, O. Gobert, G. Petite, Y. Izawa, J.F. Wagner, Ablation threshold dependence on pulse duration for copper, Appl. Surf. Sci., 197 (2002) 862-867.

[318] T. Sokollik, M. Schnurer, S. Ter-Avetisyan, P.V. Nickles, E. Risse, M. Kalashnikov, W. Sandner, G. Priebe, M. Amin, T. Toncian, O. Willi, A.A. Andreev, Transient electric fields in laser plasmas observed by proton streak 
deflectometry, Appl. Phys. Lett., 92 (2008) 091503.

[319] K. Quinn, P.A. Wilson, C.A. Cecchetti, B. Ramakrishna, L. Romagnani, G. Sarri, L. Lancia, J. Fuchs, A. Pipahl, T. Toncian, O. Willi, R.J. Clarke, D. Neely, M. Notley, P. Gallegos, D.C. Carroll, M.N. Quinn, X.H. Yuan, P. McKenna, T.V. Liseykina, A. Macchi, M. Borghesi, Laser-driven ultrafast field propagation on solid Surfaces, Phys. Rev. Lett., 102 (2009) 194801.

[320] F. Abicht, J. Braenzel, G. Priebe, C. Koschitzki, A.A. Andreev, P.V. Nickles, W. Sander, M. Schnurer, Tracing dynamics of laser-induced fields on ultrathin foils using complementary imaging with streak deflectometry, Phys. Rev. Accel. Beams, 19 (2016) 091302.

[321] M.E. Monge, G.A. Harris, P. Dwivedi, F.M. Fernandez, Mass spectrometry: recent advances in direct open air surface sampling/ionization, Chem. Rev., 113 (2013) 2269-2308.

[322] L.A. McDonnell, R.M.A. Heeren, Imaging mass spectrometry, Mass Spectrom. Rev., 26 (2007) 606-643.

[323] N. Bityurin, B.S. Luk'yanchuk, M.H. Hong, T.C. Chong, Models for laser ablation of polymers, Chem. Rev., 103 (2003) 519-552.

[324] D.B. Chrisey, A. Pique, R.A. McGill, J.S. Horwitz, B.R. Ringeisen, D.M. Bubb, P.K. Wu, Laser deposition of polymer and biomaterial films, Chem. Rev., 103 (2003) 553-576.

[325] S. Georgiou, F. Hillenkamp, Introduction: Laser ablation of molecular substrates, Chem. Rev., 103 (2003) 317-319.

[326] T. Lippert, J.T. Dickinson, Chemical and spectroscopic aspects of polymer ablation: Special features and novel directions, Chem. Rev., 103 (2003) 453-485.

[327] G. Paltauf, P.E. Dyer, Photomechanical processes and effects in ablation, Chem. Rev., 103 (2003) 487-518.

[328] L.V. Zhigilei, E. Leveugle, B.J. Garrison, Y.G. Yingling, M.I. Zeifman, Computer simulations of laser ablation of molecular substrates, Chem. Rev., 103 (2003) 321-347.

[329] M. Lenner, A. Kaplan, R.E. Palmer, Nanoscopic Coulomb explosion in ultrafast graphite ablation, Appl. Phys. Lett., 90 (2007) 153119.

[330] M. Lenner, A. Kaplan, C. Huchon, R.E. Palmer, Ultrafast laser ablation of graphite, Phys. Rev. B, 79 (2009) 184105 .

[331] A. Kaplan, M. Lenner, R.E. Palmer, Emission of ions and charged clusters due to impulsive Coulomb explosion in ultrafast laser ablation of graphite, Phys. Rev. B, 76 (2007) 073401.

[332] M. Hashida, H. Mishima, S. Tokita, S. Sakabe, Non-thermal ablation of expanded polytetrafluoroethylene with an intense femtosecond-pulse laser, Opt. Express, 17 (2009) 13116-13121.

[333] M. Murakami, R. Mizoguchi, Y. Shimada, T. Yatsuhashi, N. Nakashima, Ionization and fragmentation of anthracene with an intense femtosecond laser pulse at $1.4 \mu \mathrm{m}$, Chem. Phys. Lett., 403 (2005) 238-241.

[334] T. Yatsuhashi, N. Nakashima, Ionization of anthracene followed by fusion in the solid phase under intense nonresonant femtosecond laser fields, J. Phys. Chem. C, 113 (2009) 11458-11463.

[335] T. Yatsuhashi, N. Nakashima, Explosive desorption and fragmentation of molecular ion from solid fullerene by intense nonresonant femtosecond laser pulses, J. Phys. Chem. A, 112 (2008) 5781-5785.

[336] Y. Morimoto, R. Kanya, K. Yamanouchi, Laser-assisted electron diffraction for femtosecond molecular imaging, J. Chem. Phys., 140 (2014) 064201. 